ALEA, Lat. Am. J. Probab. Math. Stat. 14, 751-798 (2017)

DOI: 10.30757/ALEA.v14-36

\title{
Markov chains in a stratified environment
}

\section{Julien Brémont}

Laboratoire d'Analyse et de Mathématiques Appliquées, Université Paris-Est, Faculté des Sciences et Technologies, 61, avenue du Général de Gaulle, 94010 Créteil Cedex, FRANCE

E-mail address: julien.bremont@u-pec.fr

Abstract. We establish a recurrence criterion for a model of inhomogeneous random walk in $\mathbb{Z}^{d+1}$ in environment stratified by parallel affine hyperplanes. The asymptotics of the random walk is governed by some notion of directional flux variance, describing the dispersive power of some associated average flow. Some examples are presented, as well as a geometric interpretation of the criterion, in relation with the level lines of some diffusion picture.

\section{Introduction}

An important problem in the study of the asymptotical behaviour of Markov chains on a lattice concerns the recurrence/transience of random walks in an heterogeneous environment. We focus in this article on the case where the transition laws depend on a single coordinate, extending some former work (Brémont, 2016). Examples of planar random walks of this type, in environment with oriented horizontal lines, were first proposed by Campanino and Petritis (2003) in 2003, as simplified probabilistic versions of PDE transport models in stratified porous media by Matheron and De Marsily (1980).

Let us detail the model. We consider a Markov chain $\left(S_{n}\right)_{n \geq 0}$ in $\mathbb{Z}^{d} \times \mathbb{Z}$, with $d \geq 1$, starting at 0 . We write $S_{n}=\left(S_{n}^{1}, S_{n}^{2}\right) \in \mathbb{Z}^{d} \times \mathbb{Z}$. Quantities relative to the second coordinate are said "vertical". The family of transitions laws is assumed to be stratified with respect to the affine hyperplanes $\left(\mathbb{Z}^{d} \times\{n\}\right)_{n \in \mathbb{Z}}$, without hypothesis on the relative dependence between distinct levels. For the whole text, we fix Euclidean Norms and denote scalar product by a dot. Vectors are written in columns and $A^{t}$ is the transpose of the matrix $A$.

For each vertical $n \in \mathbb{Z}$, assume to be given reals $p_{n}, q_{n}, r_{n}$ with $p_{n}+q_{n}+r_{n}=1$ and a probability measure $\mu_{n}$ with support in $\mathbb{Z}^{d}$. We suppose satisfied the following conditions:

Received by the editors March 15th, 2017; accepted August 6th, 2017.

2010 Mathematics Subject Classification. 60J10, 60K20.

Key words and phrases. Markov chain, recurrence criterion, continued fraction, anisotropic pseudosphere. 
Hypotheses 1.1. For some $\delta>0$ and all $n \in \mathbb{Z}$ :

1) $\min \left\{p_{n}, q_{n}, r_{n}\right\} \geq \delta$,

2) $\sum_{k \in \mathbb{Z}^{d}}\|k\|^{\max (d, 3)} \mu_{n}(k) \leq 1 / \delta$,

3) the eigenvalues of the real symmetric matrix $\sum_{k \in \mathbb{Z}^{d}} k k^{t} \mu_{n}(k)$ are $\geq \delta$.

The last condition can be equivalently rewritten as:

$$
\sum_{k \in \mathbb{Z}^{d}}(t . k)^{2} \mu_{n}(k) \geq \delta\|t\|^{2}, t \in \mathbb{R}^{d} .
$$

In particular the subgroup of $\left(\mathbb{Z}^{d},+\right)$ generated by $\operatorname{supp}\left(\mu_{n}\right)$ is $d$-dimensional. The transition laws are then defined, for all $(m, n) \in \mathbb{Z}^{d} \times \mathbb{Z}$ and $k \in \mathbb{Z}^{d}$, by:

$$
\mathbb{P}_{(m, n),(m, n+1)}=p_{n}, \mathbb{P}_{(m, n),(m, n-1)}=q_{n}, \mathbb{P}_{(m, n),(m+k, n)}=r_{n} \mu_{n}(k) .
$$

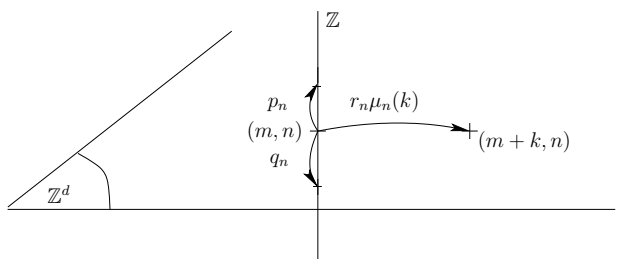

The model of Campanino and Petritis (2003) corresponds to taking $d=1$, with $p_{n}=q_{n}=p \in(0,1)$ and $\mu_{n}=\delta_{\varepsilon_{n}}$, fixing some sequence $\left(\varepsilon_{n}\right)_{n \in \mathbb{Z}}$ of \pm 1 modeling the orientation of the horizontal lines. Campanino and Petritis show the recurrence of the random walk when $\varepsilon_{n}=(-1)^{n}$ and its transience for $\varepsilon_{n}=1_{n \geq 0}-1_{n<0}$ or when the $\left(\varepsilon_{n}\right)$ are typical realizations of i.i.d. random variables with law $\left(\delta_{1}+\right.$ $\left.\delta_{-1}\right) / 2$. Staying close to this setting, several variations, extensions and second order questions were subsequently considered by different authors; see for example Campanino and Petritis (2014) for a review. For the model introduced above in the case $d=1$, under the local vertical symmetries $p_{n}=q_{n}, n \in \mathbb{Z}$, a complete characterization of the qualitative asymptotics of the random walk was recently given in Brémont (2016). A corollary of the study is that in this family of random walks, simple planar random walk, hardly recurrent, is the most recurrent one. This explains the prevalence of transience results in the litterature on the CampaninoPetritis model. For example, a growth condition larger than $\log n$ on $\varepsilon_{1}+\cdots+\varepsilon_{n}$ is sufficient to ensure transience.

Pushing to some natural limit the method used in Brémont (2016), we establish in the present article a recurrence criterion for the model described above. This furnishes a large class of recurrent random walks in $\mathbb{Z}^{2}$ and $\mathbb{Z}^{3}$. The mechanism governing the asymptotical behaviour of the random walk incidentally reveals some familiarity with classical Electromagnetism, involving notions such as flux variations. The latter represent the dispersive properties of some horizontal average flow associated with the random walk. Variations are measured in a probabilistic way, via empirical variances. We finally present some examples and provide a geometrical interpretation of the recurrence criterion, leading to considering the volume of some kind of anisotropic pseudosphere when $d=2$.

\section{Statement of the result}

Let us fix some notations for the sequel. 


\section{Definition 2.1.}

1) For $n \in \mathbb{Z}$, let $m_{n}=\sum_{k \in \mathbb{Z}^{d}} k \mu_{n}(k)$ be the expectation of $\mu_{n}$.

2) For $n \in \mathbb{Z}$, set:

$$
p_{n}^{\prime}=\frac{p_{n}}{p_{n}+q_{n}}, q_{n}^{\prime}=\frac{q_{n}}{p_{n}+q_{n}}, a_{n}=\frac{q_{n}^{\prime}}{p_{n}^{\prime}}=\frac{q_{n}}{p_{n}} \text { and } b_{n}=\frac{1}{p_{n}^{\prime}}=1+a_{n} .
$$

3) Set:

$$
\rho_{n}=\left\{\begin{array}{cc}
a_{1} \cdots a_{n}, & n \geq 1, \\
1, & n=0 \\
\left(1 / a_{n+1}\right) \cdots\left(1 / a_{-1}\right)\left(1 / a_{0}\right) & n \leq-1 .
\end{array}\right.
$$

4) For $n \geq 0$, let:

$$
v_{+}(n)=\sum_{0 \leq k \leq n} \rho_{k} \text { and } v_{-}(n)=a_{0} \sum_{-n-1 \leq k \leq-1} \rho_{k} .
$$

We denote by $\theta$ the "left shift" on indices. Given a function $f=$ $f\left(\left(p_{i}, q_{i}, r_{i}, \mu_{i}\right)_{i \in \mathbb{Z}}\right)$, we write $\theta f$ for $f\left(\left(p_{i+1}, q_{i+1}, r_{i+1}, \mu_{i+1}\right)_{i \in \mathbb{Z}}\right)$. In particular we have the cocycle relation:

$$
\forall(n, k) \in \mathbb{Z}^{2}, \rho_{n+k}=\rho_{n} \theta^{n} \rho_{k} .
$$

For two functions $f(x)$ and $g(x)$, we shall write $f \asymp g$ if there exists an absolute constant $C>0$ to that for all $x,(1 / C) f(x) \leq g(x) \leq C f(x)$.

We next define the reciprocal function of a non-negative non-decreasing function defined on the set of integers $\mathbb{N}=\{0,1, \cdots\}$.

Definition 2.2. Let $f: \mathbb{N} \rightarrow \mathbb{R} \cup\{+\infty\}$ be non-decreasing. For $x \in \mathbb{R}_{+}$, let $f^{-1}(x)=\sup \{n \in \mathbb{N} \mid f(n) \leq x\}$, with the convention that $\sup \varnothing=0$ and $\sup \mathbb{N}=$ $+\infty$

The analysis developed below runs as follows. Due to the stratification of the environment, when restricting the random walk to its vertical movements, the vertical coordinate is a Markov chain. This is a very special situation for a multidimensional inhomogeneous Markov chain. The treatment of the recurrence/transience of the vertical coordinate is standard and we rapidly focus on the case when the vertical component is recurrent. In this case and when $d=1$, the only possible direction of escape is the horizontal one. We consider the sequence of return times on the horizontal axis, which is an i.i.d. random walk with heavy-tailed jump. Informally, considering this sub-random walk appears to be equivalent to considering the properties of the flux of some horizontal flow. When $d \geq 1$, this flow lies in $\mathbb{Z}^{d}$ and can be desintegrated in directional fluxes.

Let us now introduce some definitions concerning the variations of directional fluxes. These may be considered as directional macrodispersion coefficients. Related longitudinal macrodispersion coefficients already appear since a long time in Physics, see for example in Matheron and De Marsily (1980), when $d=1$.

\section{Definition 2.3.}

1) Let $S_{+}^{d-1}=\left\{x \in \mathbb{R}^{d} \mid\|x\|=1, x_{1} \geq 0\right\}$ be a half unit Euclidean sphere of $\mathbb{R}^{d}$. It is a compact space. We naturally write $d u$ for standard Lebesgue measure on $S_{+}^{d-1}$. 
2) Define for $n \geq 0$ the structure function, depending only on the vertical:

$$
\Phi_{s t r}(n)=\left(n \sum_{-v_{-}^{-1}(n) \leq k \leq v_{+}^{-1}(n)} \frac{1}{\rho_{k}}\right)^{1 / 2} .
$$

3) For $u \in S_{+}^{d-1}$ and $m \geq 0, n \geq 0$, introduce:

$$
\Phi_{u}(-m, n)=\left(\sum_{-v_{-}^{-1}(m) \leq k \leq l \leq v_{+}^{-1}(n)} \rho_{k} \rho_{l}\left[\frac{1}{\rho_{k}^{2}}+\frac{1}{\rho_{l}^{2}}+\left(\sum_{s=k}^{l} \frac{r_{s} m_{s} \cdot u}{p_{s} \rho_{s}}\right)^{2}\right]\right)^{1 / 2} \in[0,+\infty] .
$$

For $n \geq 0$, set $\Phi_{u}(n)=\Phi_{u}(-n, n)$ and $\Phi_{u,+}(n)=\sqrt{\Phi_{u}^{2}(-n, 0)+\Phi_{u}^{2}(0, n)}$.

The essential aim of the article is to prove the following result.

Theorem 2.4. The random walk is recurrent if and only if:

$$
\sum_{n \geq 1} n^{-d-1} \int_{S_{+}^{d-1}} \frac{\left(\Phi_{u}^{-1}(n)\right)^{2}}{\Phi_{u,+}^{-1}(n)} d u=+\infty .
$$

The meaning of the criterion will be clarified in the last section. For each fixed $u \in S_{+}^{d-1}$ the general term appearing above is related to a diffusion property of the level lines of the map $(m, n) \longmapsto \Phi_{u}(-m, n)$, with $m \geq 0, n \geq 0$. When $d=2$, the global integral is essentially the volume in $\mathbb{R}^{3}$ of an object looking like some two-sided top.

We shall deduce the following consequences, making the recurrence criterion easier to use in many classical situations.

\section{Proposition 2.5.}

1) A sufficient condition for transience is:

$$
\sum_{n \geq 1} \int_{S_{+}^{d-1}}\left(\Phi_{u}(n)\right)^{-d} d u<+\infty .
$$

The latter is satisfied when $\sum_{n \geq 1}\left(\Phi_{s t r}(n)\right)^{-d}<+\infty$. There is thus transience when:

i) $d \geq 3$.

ii) $d=2$ and $\Phi_{\text {str }}(n) \geq \sqrt{n}(\log n)^{1 / 2+\varepsilon}$. This is true if $p_{n}=q_{n}, n \in \mathbb{Z}$.

iii) $d=1$ and $\Phi_{\text {str }}(n) \geq n(\log n)^{1+\varepsilon}$.

2) When $m_{n}=0$ for all $n \in \mathbb{Z}$, the random walk is transient if and only if:

$$
\sum_{n \geq 1}\left(\Phi_{s t r}(n)\right)^{-d}<+\infty .
$$

3) (Antisymmetry). When $\left(p_{-n}, q_{-n}, r_{-n}, m_{-n}\right)=\left(q_{n}, p_{n}, r_{n},-m_{n}\right)$, for $n \geq 0$ (in particular $m_{0}=0$ and $\left.p_{0}=q_{0}\right)$, the random walk is transient if and only if:

$$
\sum_{n \geq 1} \int_{S_{+}^{d-1}}\left(\Phi_{u}(0, n)\right)^{-d} d u<+\infty
$$


Remark 2.6. The vertical coordinate (restricting to vertical movements) is the Markov chain on $\mathbb{Z}$ with jump probabilities $\mathbb{P}_{n, n+1}=p_{n}^{\prime}$ and $\mathbb{P}_{n, n-1}=q_{n}^{\prime}$. For an observer at 0 of this one-dimensional random walk, the quantity $\rho_{n}$ can be considered as the level of the sea at site $n \in \mathbb{Z}$. The global effort for going from 0 to $n$ is somehow measured by $v_{+}(n)$. This is some kind of asymmetric distance between 0 and $n$. The point $v_{+}^{-1}(n)$ is somehow the point at distance $n$ from 0 for some new metric. It is classical (we reprove it below) that this random walk is recurrent if and only if $v_{+}(n) \rightarrow+\infty$ and $v_{-}(n) \rightarrow+\infty$, as $n \rightarrow+\infty$, meaning that the effort for going either North or South is infinite. This may also be seen classically by the fact that this one-dimensional random walk is reversible; for example the quantity $v_{+}(n)$ is the effective resistance from 0 to $n$. More important, $1 / \rho_{n}$ is reminiscent of some invariant measure. We shall interpret it as a conductivity, acting multiplicatively. When $\rho_{n}$ is small then $1 / \rho_{n}$ is large. The quantity $r_{s} m_{s} \cdot u /\left(p_{s} \rho_{s}\right)$ in $\Phi_{u}(-m, n)$ then essentially corresponds to what flows in the horizontal direction $u$ at the vertical $s \in \mathbb{Z}$. The main contributions in $\Phi_{u}(-m, n)$ correspond to deep valleys: $k$ and $l$ with large $\rho_{k}$ and $\rho_{l}$ and some $u \in[k, l]$ with small $\rho_{u}$.

Remark 2.7. We shall discuss in section 7 the case when $\sum_{n \in \mathbb{Z}}\left(1 / \rho_{n}\right)<+\infty$. The random walk is then pushed very strongly towards the horizontal subspace $\mathbb{Z}^{d}$ and the vertical component (restricted to vertical jumps) is positive recurrent. As we shall see, when $d=1$, the recurrence/transience is decided by the value of a single number.

Remark 2.8. In the present setting, $\left(v_{+}(n)\right)_{n \geq 0}$ and $\left(v_{-}(n)\right)_{n \geq 0}$ can be bounded. In the first case, with our convention, this implies that $v_{+}^{-1}(n)=+\infty$ for $n$ large enough. As for $k=l$, the term $\rho_{k} \rho_{l}\left(1 / \rho_{k}^{2}+1 / \rho_{l}^{2}\right)$ has order at least one, $\Phi_{u}(n)$ and $\Phi_{u,+}(n)$ are equal to $+\infty$ for $n$ large enough, uniformly in $u \in S_{+}^{d-1}$. The same is true for $\Phi_{s t r}(n)$, since $\rho_{k} \rightarrow 0$, as $k \rightarrow+\infty$, in this case. We come back on this later.

Remark 2.9. When $m_{n}=0$ for all $n \in \mathbb{Z}$, then $\Phi_{u}(n)$ and $\Phi_{u,+}(n)$ do not depend on $u \in S_{+}^{d-1}$ and essentially equal $\Phi_{s t r}(n)$, which can be considered as a structure function independent of what may flow horizontally. In the general case, both $\Phi_{u}(n)$ and $\Phi_{u,+}(n)$ are always larger than $\Phi_{s t r}(n)$.

Remark 2.10. Using the classical formula for the variance of a random variable $Z$, $\operatorname{Var}(Z)=\mathbb{E}\left(\left(Z-Z^{\prime}\right)^{2}\right) / 2$, where $Z^{\prime}$ is independent of $Z$ and with the same law, we observe that:

$$
\sum_{0 \leq k \leq l \leq v_{+}^{-1}(n)} \rho_{k} \rho_{l}\left[\left(\sum_{s=k}^{l} \frac{r_{s} m_{s} \cdot u}{p_{s} \rho_{s}}\right)^{2}\right] \asymp n^{2} \mathbb{V} a r\left(\sum_{s=0}^{X} \frac{r_{s} m_{s} \cdot u}{p_{s} \rho_{s}}\right),
$$

when $\left(r_{s} m_{s} \cdot u /\left(p_{s} \rho_{s}\right)\right)_{s}$ is considered as a fixed sequence and $X$ is a random integer in $\left[0, v_{+}^{-1}(n)\right]$, with density proportional to $\rho_{k}$. The same is true for the second part of $\Phi_{u}^{2}(n)$ in $\left[-v_{-}^{-1}(n), v_{+}^{-1}(n)\right]$, when using cocycles notations (that we haven't introduced).

Plan of the article. In section 3, we develop preliminaries about a special type of complex continued fractions that will be central in this text. We next discuss the asymptotics of the vertical component. In section 4, reducing the analysis to the case when the vertical component is recurrent, we focus on the sequence 
of return times on $\mathbb{Z}^{d}$. This leads to considering some i.i.d. heavy-tailed random walk. For such a random walk a recurrence is available, the strong Chung-Fuchs recurrence criterion. This requires the estimation of the behaviour at the origin of some characteristic function. A preliminary analysis is started at the end of section 4 , where this characteristic function is naturally developed in continued fraction. Detailed computations are completed in section 5. In section 6, with all ingredients in hand we make the proof of theorem 2.4 and proposition 2.5. In section 7, we

detail concrete examples, discuss some variations in the hypotheses and present a geometrical interpretation of the recurrence criterion.

\section{Preliminaries}

3.1. Sleszynski-Pringsheim continued fractions. In the sequel, a general formal finite continued fraction is written as follows:

$$
\left[\left(c_{1}, d_{1}\right) ;\left(c_{2}, d_{2}\right) ; \cdots ;\left(c_{n}, d_{n}\right)\right]=\frac{c_{1}}{d_{1}+\frac{c_{2}}{d_{2}+\frac{\cdots}{\cdots+\frac{c_{n}}{d_{n}}}}}
$$

We shall consider in this article SP-continued fractions (for SleszynskiPringsheim, cf Beardon and Lorentzen, 2001). Finite SP-continued fractions correspond to finitely many applications to some $z_{0} \in \mathbb{C}$ with $|z| \leq 1$ of maps of the form $z \longmapsto c /(d+z)$, with complex numbers $c \neq 0$ and $d$ so that $|c|+1 \leq|d|$, hence preserving the unit disk. Finite SP-continued are therefore well defined. Infinite SP-continued fractions, written as:

$$
\left[\left(c_{1}, d_{1}\right) ;\left(c_{2}, d_{2}\right) ; \cdots\right]=\frac{c_{1}}{d_{1}+\frac{c_{2}}{d_{2}+\cdots}}
$$

also converge. This is the Sleszynski-Pringsheim theorem (see Lorentzen and Waadeland, 1992), essentially reproved below.

Let us recall some classical facts from the theory of continued fractions. Formally, for all $n \geq 0$, any finite continued fraction can be reduced as:

$$
\left[\left(c_{1}, d_{1}\right) ;\left(c_{2}, d_{2}\right) ; \cdots ;\left(c_{n}, d_{n}\right)\right]=\frac{A_{n}}{B_{n}} .
$$

The $\left(A_{n}\right)$ and $\left(B_{n}\right)$ satisfy the same recursive relation, with different initial data:

$$
\left\{\begin{array}{l}
A_{n}=d_{n} A_{n-1}+c_{n} A_{n-2}, n \geq 1, \text { with } A_{-1}=1, A_{0}=0 \\
B_{n}=d_{n} B_{n-1}+c_{n} B_{n-2}, n \geq 1, \text { with } B_{-1}=0, B_{0}=1
\end{array}\right.
$$

We shall require the following classical determinant. For $n \geq 1$ :

$$
A_{n} B_{n-1}-A_{n-1} B_{n}=(-1)^{n+1} c_{1} \cdots c_{n} .
$$

This is a consequence of the relation $A_{n} B_{n-1}-A_{n-1} B_{n}=\left(-c_{n}\right)\left(A_{n-1} B_{n-2}-\right.$ $\left.A_{n-2} B_{n-1}\right)$, for $n \geq 1$, and the initial data of $\left(A_{n}\right)$ and $\left(B_{n}\right)$. This furnishes a 
representation as a series:

$$
\left[\left(c_{1}, d_{1}\right) ;\left(c_{2}, d_{2}\right) ; \cdots ;\left(c_{n}, d_{n}\right)\right]=\frac{A_{n}}{B_{n}}=\sum_{k=1}^{n}\left(\frac{A_{k}}{B_{k}}-\frac{A_{k-1}}{B_{k-1}}\right)=\sum_{k=1}^{n} \frac{(-1)^{k+1} c_{1} \cdots c_{k}}{B_{k} B_{k-1}} .
$$

A particular class of SP-continued fractions will appear frequently in the text. We state a general lemma to which we shall often refer to.

Lemma 3.1. Assume that $v_{+}(n) \rightarrow+\infty$, as $n \rightarrow+\infty$.

(1) Let sequences of complex numbers $\left(\gamma_{n}\right)_{n \geq 1}$ and $\left(\gamma_{n}^{\prime}\right)_{n \geq 1}$ be such that $0<$ $\left|\gamma_{n}\right| \leq 1,\left|\gamma_{n}^{\prime}\right| \leq 1$. Then the following is a well-defined SP-continued fraction:

$\left[\left(a_{1}, b_{1} / \gamma_{1}\right) ;\left(-a_{2}, b_{2} / \gamma_{2}\right) ; \cdots ;\left(-a_{n-1}, b_{n-1} / \gamma_{n-1}\right) ;\left(-a_{n}, b_{n} / \gamma_{n}-\gamma_{n}^{\prime}\right)\right]$

As $n \rightarrow+\infty$, it converges to the infinite SP-continued fraction:

$$
\left[\left(a_{1}, b_{1} / \gamma_{1}\right) ;\left(-a_{2}, b_{2} / \gamma_{2}\right) ; \cdots ;\left(-a_{n}, b_{n} / \gamma_{n}\right) ; \cdots\right]
$$

Moreover, the latter is the limit of $A_{n} / B_{n}$, as $n \rightarrow+\infty$, where:

$$
\left\{\begin{array}{c}
A_{n}=\frac{b_{n}}{\gamma_{n}} A_{n-1}-a_{n} A_{n-2}, n \geq 2, \text { with } A_{-1}=1, A_{0}=0, A_{1}=a_{1}, \\
B_{n}=\frac{b_{n}}{\gamma_{n}} B_{n-1}-a_{n} B_{n-2}, n \geq 2, \text { with } B_{-1}=0, B_{0}=1, B_{1}=b_{1} / \gamma_{1} .
\end{array}\right.
$$

(2) Let $v_{+}(-1)=0$. Then the solutions $\left(B_{n}\right)$ of (3.4) check:

$$
\left|B_{n}\right|-\left|B_{n-1}\right| \geq a_{n}\left(\left|B_{n-1}\right|-\left|B_{n-2}\right|\right), n \geq 1 \text {. }
$$

As a result:

$$
\left|B_{n}\right| \geq v_{+}(n), n \geq-1 .
$$

If the $0<\gamma_{n} \leq 1$ are reals, then $B_{n}>B_{n-1}>\cdots>B_{-1}=0$. When $\gamma_{n}=1, n \geq 1$, then:

$$
B_{n}=v_{+}(n), n \geq-1 \text { and } A_{n}=v_{+}(n)-1, n \geq 0
$$

(3) In (3.4), the map $n \longmapsto\left|B_{n}\right| / v_{+}(n), n \geq 0$, is non-decreasing. Also:

$$
\sum_{k>n} \frac{\rho_{k}}{\left|B_{k} B_{k-1}\right|} \leq \frac{v_{+}(n)}{\left|B_{n}\right|^{2}} \leq \frac{1}{\left|B_{n}\right|}, \text { for } n \geq 0 .
$$

Proof: As a preliminary remark, observe that the solutions $\left(B_{k}\right)$ of the second recursive relation in (3.4) check $\left|B_{n}\right| \geq b_{n}\left|B_{n-1}\right|-a_{n}\left|B_{n-2}\right|, n \geq 1$. Hence:

$$
\left|B_{n}\right|-\left|B_{n-1}\right| \geq a_{n}\left(\left|B_{n-1}\right|-\left|B_{n-2}\right|\right), n \geq 1 \text {. }
$$

Iterating, we get $\left|B_{n}\right|-\left|B_{n-1}\right| \geq \rho_{n}$. Thus $\left|B_{n}\right| \geq v_{+}(n)$, for all $n \geq-1$.

In point 1., the finite SP-continued fraction is well-defined because $a_{k} \neq 0$, $\left|b_{k} / \gamma_{k}\right|-a_{k} \geq b_{k}-a_{k}=1$ and $\left|\gamma_{n}^{\prime}\right| \leq 1$ for the last term. From the system (3.1), the system (3.4) is verified. Then via (3.3), using that the $k^{t h}$ determinant is $\rho_{k}$ :

$$
\left[\left(a_{1}, b_{1} / \gamma_{1}\right) ;\left(-a_{2}, b_{2} / \gamma_{2}\right) ; \cdots ;\left(-a_{n}, b_{n} / \gamma_{n}-\gamma_{n}^{\prime}\right)\right]=\sum_{k=1}^{n-1} \frac{\rho_{k}}{B_{k} B_{k-1}}+\frac{\rho_{n}}{B_{n-1} \tilde{B}_{n}},
$$


where $\tilde{B}_{n}=\left(b_{n} / \gamma_{n}-\gamma_{n}^{\prime}\right) B_{n-1}-a_{n} B_{n-2}$. We get:

$$
\left|\tilde{B}_{n}\right| \geq\left(b_{n}-1\right)\left|B_{n-1}\right|-a_{n}\left|B_{n-2}\right| \geq a_{n}\left(\left|B_{n-1}\right|-\left|B_{n-2}\right|\right) \geq a_{n} \rho_{n-1}=\rho_{n} .
$$

In (3.5), observe first that the first term in the right-hand side is absolutely convergent. Indeed, using that $\left|B_{k}\right| \geq v_{+}(k)$ :

$$
\sum_{k \geq 1} \frac{\rho_{k}}{\left|B_{k} B_{k-1}\right|} \leq \sum_{k \geq 1} \frac{\rho_{k}}{v_{+}(k) v_{+}(k-1)}=\sum_{k \geq 1}\left(\frac{1}{v_{+}(k-1)}-\frac{1}{v_{+}(k)}\right)=1 .
$$

As $\left|B_{n-1}\right| \geq v_{+}(n-1) \rightarrow+\infty$ and $|\bar{B}| \geq \rho_{n}$, the second term in the right-hand side in (3.5) converges to 0 , so the right-hand side converges to $\sum_{k>1} \rho_{k} /\left(B_{k} B_{k-1}\right)$.

To complete point 2., suppose that the $0<\gamma_{n} \leq 1$ are real. We have:

$$
B_{n}-B_{n-1}=\frac{\left(1-\gamma_{n}\right) b_{n}}{\gamma_{n}} B_{n-1}+a_{n}\left(B_{n-1}-B_{n-2}\right), n \geq 2
$$

The condition " $B_{n}>B_{n-1} \geq 0$ " is then transmitted recursively. If $\gamma_{n}=1$, then $B_{n}-B_{n-1}=a_{n}\left(B_{n-1}-B_{n-2}\right)$, giving $B_{n}-B_{n-1}=\rho_{n}$ and thus $B_{n}=v_{+}(n)$, $n \geq 0$. Similarly:

$$
A_{n}-A_{n-1}=a_{n}\left(A_{n-1}-A_{n-2}\right)=\cdots=a_{n} \cdots a_{2}\left(A_{1}-A_{0}\right)=\rho_{n} .
$$

As $A_{0}=0$, we obtain $A_{n}=v_{+}(n)-1, n \geq 0$.

Concerning point 3., we first show that $n \longmapsto\left|B_{n}\right| / v_{+}(n), n \geq 0$, is nondecreasing. We will require it in the equivalent form $\left|B_{n}\right| /\left(\left|B_{n+1}\right|-\left|B_{n}\right|\right) \leq$ $v_{+}(n) /\left(v_{+}(n+1)-v_{+}(n)\right)$. Write:

$$
\begin{aligned}
v_{+}(n)\left|B_{n+1}\right|-v_{+}(n+1)\left|B_{n}\right| & \geq v_{+}(n)\left(b_{n+1}\left|B_{n}\right|-a_{n+1}\left|B_{n-1}\right|\right)-v_{+}(n+1)\left|B_{n}\right| \\
& \geq a_{n+1}\left(v_{+}(n-1)\left|B_{n}\right|-v_{+}(n)\left|B_{n-1}\right|\right) \\
& \geq \cdots \geq \rho_{n+1}\left(\left|B_{0}\right| v_{+}(-1)-v_{+}(0)\left|B_{-1}\right|\right)=0 .
\end{aligned}
$$

For the last inequalities, using the previous results, for $n \geq 0$ :

$$
\begin{aligned}
\sum_{k>n} \frac{\rho_{k}}{\left|B_{k} B_{k-1}\right|} & =\sum_{k>n} \rho_{k}\left(\frac{1}{\left|B_{k-1}\right|}-\frac{1}{\left|B_{k}\right|}\right) \frac{1}{\left|B_{k}\right|-\left|B_{k-1}\right|} \\
& \leq \sum_{k>n} \rho_{k}\left(\frac{1}{\left|B_{k-1}\right|}-\frac{1}{\left|B_{k}\right|}\right) \frac{1}{a_{k} \cdots a_{n+2}\left(\left|B_{n+1}\right|-\left|B_{n}\right|\right)} \\
& \leq \frac{\rho_{n+1}}{\left|B_{n+1}\right|-\left|B_{n}\right|} \sum_{k>n}\left(\frac{1}{\left|B_{k-1}\right|}-\frac{1}{\left|B_{k}\right|}\right) \\
& \leq \frac{v_{+}(n+1)-v_{+}(n)}{\left|B_{n+1}\right|-\left|B_{n}\right|} \frac{1}{\left|B_{n}\right|} \leq \frac{v_{+}(n)}{\left|B_{n}\right|^{2}} .
\end{aligned}
$$

This completes the proof of the lemma.

3.2. Behavior of the vertical component. Let us first focus on the vertical component of the random walk. As already mentioned, when restricting the random walk to the subsequence of its vertical movements, the vertical component is a Markov Chain on $\mathbb{Z}$ with transition probabilities $\mathbb{P}_{n, n-1}=q_{n}^{\prime}$ and $\mathbb{P}_{n, n+1}=p_{n}^{\prime}, n \in \mathbb{Z}$. The question of its recurrence/transience is classical in the study of birth and death processes. 
Lemma 3.2. Let the Markov chain on $\mathbb{Z}$ with transition probabilities $\mathbb{P}_{n, n+1}=p_{n}^{\prime}$ and $\mathbb{P}_{n, n-1}=q_{n}^{\prime}, n \in \mathbb{Z}$. Then it is recurrent if and only if $v_{+}(n) \rightarrow+\infty$ and $v_{-}(n) \rightarrow+\infty$, as $n \rightarrow+\infty$.

Proof: Fix $N>1$ and let $f(k)=\mathbb{P}_{k}$ (exit $[0, N]$ on the left side), $0 \leq k \leq N$. By the Markov property, $k \longmapsto f(k)$ is harmonic, so for $1 \leq k \leq N-1$ :

$$
f(k)=p_{k}^{\prime} f(k+1)+q_{k}^{\prime} f(k-1) .
$$

Let $g(k)=f(k)-f(k-1), 1 \leq k \leq N$. We obtain $g(k)=\left(p_{k} / q_{k}\right) g(k+1)$ and therefore $g(k)=\rho_{k-1} g(1), 1 \leq k \leq N$. As a result:

$$
-1=\sum_{k=1}^{N} g(k)=-\mathbb{P}_{1}(\text { exit }[0, N] \text { at } N) \sum_{1 \leq k \leq N} \rho_{k-1} .
$$

Hence $\mathbb{P}_{1}($ reach 0$)=1 \Leftrightarrow \lim _{n \rightarrow+\infty} v_{+}(n)=+\infty$. In the same way $\mathbb{P}_{-1}($ reach 0$)=$ $1 \Leftrightarrow \lim _{n \rightarrow+\infty} v_{-}(n)=+\infty$. This furnishes the desired result.

We shall use the previous criterion in a reformulation using trees. We say that a random variable $X$ has the geometrical law $\mathcal{G}(p), 0<p<1$, if $\mathbb{P}(X=n)=p^{n}(1-p)$, $n \geq 0$.

Lemma 3.3. Let $\left(Z_{n}^{+}\right)_{n \geq 1}$ be the Galton-Watson tree with $Z_{1}^{+}=1$ and such that the law of the number of children at level $n+1$ of an individual at level $n \geq 1$ is $\mathcal{G}\left(p_{n}^{\prime}\right)$, independently. Then this tree is finite almost-surely if and only if $v_{+}(n) \rightarrow$ $+\infty$, as $n \rightarrow+\infty$.

Proof: Since $\left\{Z_{n}^{+}=0\right\} \subset\left\{Z_{n+1}^{+}=0\right\}$, the almost-sure finiteness is equivalent to $\mathbb{P}\left(Z_{n}^{+}=0\right) \rightarrow 1$, as $n \rightarrow+\infty$. Fix $0<s<1$ and note that:

$$
\mathbb{E}\left(s^{Z_{n}^{+}}\right)-s \leq \mathbb{P}\left(Z_{n}^{+}=0\right) \leq \mathbb{E}\left(s^{Z_{n}^{+}}\right) .
$$

Taking $n \geq 2$, the construction of the Galton-Watson tree implies that:

$$
\mathbb{E}\left(s^{Z_{n}^{+}}\right)=\mathbb{E}\left[\left(\frac{1-p_{n-1}^{\prime}}{1-s p_{n-1}^{\prime}}\right)^{Z_{n-1}^{+}}\right]=\mathbb{E}\left[\left(\frac{a_{n-1}}{b_{n-1}-s}\right)^{Z_{n-1}^{+}}\right] .
$$

Iterating the procedure, i.e. using $a_{n-1} /\left(b_{n-1}-s\right)$ in place of $s$, we obtain the following SP-continued fraction:

$$
\mathbb{E}\left(s^{Z_{n}^{+}}\right)=\left[\left(a_{1}, b_{1}\right) ;\left(-a_{2}, b_{2}\right) ; \cdots ;\left(-a_{n-2}, b_{n-2}\right) ;\left(-a_{n-1}, b_{n-1}-s\right)\right] .
$$

This corresponds to $\gamma_{k}=1$ and $\gamma_{n}^{\prime}=s$ in the first point of lemma 3.1. Lemma 3.1, relation (3.5), and the computation of the partial quotients now furnish:

$$
\mathbb{E}\left(s^{Z_{n}^{+}}\right)=\frac{v_{+}(n-2)-1}{v_{+}(n-2)}+\frac{\rho_{n-1}}{v_{+}(n-1) \tilde{B}_{n-1}},
$$

with $\tilde{B}_{n-1}=\left(b_{n-1}-s\right) v_{+}(n-2)-a_{n-1} v_{+}(n-3)$. Observe that both:

$$
\tilde{B}_{n-1} \geq \rho_{n-1} \text { and } \tilde{B}_{n-1} \geq(1-s) v_{+}(n-2) \text {. }
$$

- If $v_{+}(n) \rightarrow+\infty$, then $\mathbb{E}\left(s^{Z_{n}^{+}}\right) \rightarrow 1$ uniformly in $0<s<1$, so $\mathbb{P}\left(Z_{n}^{+}=0\right) \rightarrow 1$.

- If $v_{+}(n) \rightarrow_{n \rightarrow+\infty} b \in(0,+\infty)$, then first $\rho_{n} \rightarrow 0$. Also for fixed $0<s<1$ we have $\liminf \operatorname{in}_{n} \tilde{B}_{n-1} \geq(1-s) b>0$, so that $\mathbb{E}\left(s^{Z_{n}^{+}}\right)$tends to $(b-1) / b<1$, giving $\lim _{n} \mathbb{P}\left(Z_{n}^{+}=0\right)=(b-1) / b<1$. 
Remark 3.4. Symmetrically for the Southern direction of the vertical component, one introduces, with decreasing indices $n \leq-1$, the Galton-Watson tree $\left(Z_{n}^{-}\right)_{n \leq-1}$ with $Z_{-1}^{-}=1$ such that, independently, the law of the number of children at level $n-1$ of an individual at level $n$ is $\mathcal{G}\left(q_{n}^{\prime}\right)$. In the same way, the tree is almost-surely finite if and only if $\lim _{k \rightarrow+\infty} v_{-}(k)=+\infty$.

\section{An i.i.d. random walk in $\mathbb{Z}^{d}$}

4.1. Reduction of the problem. As a first step, we reduce the analysis to the case when the vertical component is recurrent. Indeed, the transience of the vertical component implies the transience of the random walk. From the previous section the transience of the vertical component is equivalent to the finiteness of $\lim _{n \rightarrow+\infty} v_{+}(n)$ or $\lim _{n \rightarrow+\infty} v_{-}(n)$. Suppose for example that $v_{+}(n)$ is bounded in $+\infty$. With our convention, $v_{+}^{-1}(n)=+\infty$ for $n$ large enough. Thus, for $n$ large enough, uniformly in $u \in S_{+}^{d-1}$ :

$$
\Phi_{u}^{2}(0, n) \geq \sum_{k=0}^{\infty} \rho_{k}^{2}\left(\frac{2}{\rho_{k}^{2}}\right)=+\infty .
$$

Hence, $\Phi_{u}^{-1}(n)$ and $\Phi_{u,+}^{-1}(n)$ are bounded, uniformly in $u$ and $n$. As a result the sum appearing in theorem 2.4 is less than $\sum_{n \geq 1} n^{-1-d} \times C<+\infty$, as $d \geq 1$.

We now assume for the rest of the article that the vertical component is recurrent. Equivalently, $\lim _{n \rightarrow+\infty} v_{ \pm}(n)=+\infty$. This allows to introduce the following random times $0=\sigma_{0}<\tau_{0}<\sigma_{1}<\tau_{1}<\cdots$, where, for $k \geq 0$ :

$$
\tau_{k}=\min \left\{n>\sigma_{k} \mid S_{n}^{2} \neq 0\right\}, \sigma_{k+1}=\min \left\{n>\tau_{k} \mid S_{n}^{2}=0\right\} .
$$

Define the $\mathbb{Z}^{d}$-displacement:

$$
D_{n}=S_{\sigma_{n}}^{1}-S_{\sigma_{n-1}}^{1}
$$

The key point, consequence of the fact that the environment is invariant under $\mathbb{Z}^{d}$-translations, is just that the $\left(D_{n}\right)_{n \geq 1}$ are globally independent and identically distributed. The following lemma is essentially taken from Campanino and Petritis (2003).

Lemma 4.1. Let $T_{0}=0$ and $T_{n}=D_{1}+\cdots+D_{n}, n \geq 1$. The random walk $\left(S_{n}\right)_{n \geq 0}$ is recurrent in $\mathbb{Z}^{d+1}$ if and only if $\left(T_{n}\right)_{n \geq 0}$ is recurrent in $\mathbb{Z}^{d}$.

Proof: If $\left(T_{n}\right)_{n \geq 0}$ is recurrent in $\mathbb{Z}^{d}$, then $\left(S_{n}\right)$ is recurrent in $\mathbb{Z}^{d+1}$, as $S_{\sigma_{n}}=$ $\left(T_{n}, 0\right)$. When $\left(T_{n}\right)$ is transient, the standard properties of the Green function and the invariance of the environment under $\mathbb{Z}^{d}$-translations give:

$$
\exists C, \forall x \in \mathbb{Z}^{d}, \sum_{n \geq 1} \mathbb{P}\left(T_{n}=x\right) \leq C .
$$

Let $\Gamma \sim \mathcal{G}\left(r_{0}\right)$ and $\xi_{k} \sim \mu_{0}$, for $k \geq 1$, so that $\left(\left(\xi_{k}\right)_{k \geq 1}, \Gamma\right)$ are globally independent and also independent from the sequence $\left(T_{n}\right)$. Remark that $\left(S_{l}^{1}\right)_{l \in\left[\sigma_{k}, \tau_{k}\right)}$ and $\left(T_{k}+\right.$ $\left.\sum_{1 \leq m \leq l} \xi_{m}\right)_{0 \leq l \leq \Gamma}$ have the same law. Define the real random variable:

$$
H=\sum_{1 \leq k \leq \Gamma}\left\|\xi_{k}\right\|
$$

Observe that $S_{n}$ can be 0 only for $n$ in some $\left[\sigma_{k}, \tau_{k}\right)$. Now:

$$
\mathbb{P}\left(\exists n \in\left[\sigma_{k}, \tau_{k}\right), S_{n}=0\right) \leq \mathbb{P}\left(H \geq\left\|T_{k}\right\|\right) .
$$


This provides, making use of (4.1):

$$
\begin{aligned}
\sum_{k \geq 1} \mathbb{P}\left(\exists n \in\left[\sigma_{k}, \tau_{k}\right), S_{n}=0\right) \leq \sum_{k \geq 1} \mathbb{P}\left(H \geq\left\|T_{k}\right\|\right) & \leq \sum_{x \in \mathbb{Z}^{d}} \sum_{k \geq 1} \mathbb{P}\left(T_{k}=x\right) \mathbb{P}(H \geq\|x\|) \\
& \leq C \sum_{x \in \mathbb{Z}^{d}} \mathbb{P}(H \geq\|x\|) \leq C^{\prime} \mathbb{E}\left(H^{d}\right)
\end{aligned}
$$

for another constant $C^{\prime}$. We will show that the last term is finite. By the BorelCantelli lemma, this will imply that $\left(S_{n}\right)$ is transient. We have:

$$
\begin{aligned}
\mathbb{E}\left(H^{d}\right)=\sum_{n \geq 0} \mathbb{P}(\Gamma=n) \mathbb{E}\left[\left(\sum_{1 \leq k \leq n}\left\|\xi_{k}\right\|\right)^{d}\right] & \leq\left(1-r_{0}\right) \sum_{n \geq 0} r_{0}^{n} n^{d-1} \mathbb{E}\left(\sum_{1 \leq k \leq n}\left\|\xi_{k}\right\|^{d}\right) \\
& \leq\left(1-r_{0}\right) \sum_{n \geq 0} r_{0}^{n} n^{d} \mathbb{E}\left(\left\|\xi_{1}\right\|^{d}\right)<\infty,
\end{aligned}
$$

using hypothesis 1.1 on the moments of $\mu_{0}$. This completes the proof of the lemma.

The previous lemma reduces the problem of the recurrence of $\left(S_{n}\right)$ to that of $\left(T_{n}\right)$. We have gained one dimension and the fact that $T_{n}=D_{1}+\cdots+D_{n}$, where $\left(D_{n}\right)$ are $i . i . d$. . The counterpart is that the law of $D_{1}$ is complicated and heavytailed. Set:

$$
D=D_{1} \text { and } \chi_{D}(t)=\mathbb{E}\left(e^{i t . D}\right), t \in \mathbb{R}^{d} .
$$

The following theorem gives an analytical recurrence criterion for any i.i.d. random walk in $\mathbb{Z}^{d}$, without moment conditions. It is named the strong form of the ChungFuchs recurrence criterion. For a proof, see Spitzer (1976). Recall that $S_{+}^{d-1}$ denotes the half unit sphere. We write $B_{d}(0, \eta)$ for the ball of center 0 and radius $\eta>0$ in $\mathbb{R}^{d}$.

Theorem 4.2. Let $\left(X_{n}\right)_{n>1}$ be i.i.d $\mathbb{Z}^{d}$-valued random variables such that the subgroup of $\left(\mathbb{Z}^{d},+\right)$ generated by the support of the law of $X_{1}$ is $\mathbb{Z}^{d}$. Let $S_{n}=$ $X_{1}+\cdots+X_{n}, n \geq 1$, and $\chi(t)=\mathbb{E}\left(e^{i t . X_{1}}\right), t \in \mathbb{R}^{d}$. Then the random walk $\left(S_{n}\right)_{n \geq 0}$ is transient if and only if for some $\eta>0$ :

$$
\int_{B_{d}(0, \eta)} \operatorname{Re}\left(\frac{1}{1-\chi(x)}\right) d x<+\infty .
$$

We shall apply the previous result to $\left(T_{n}\right)$. Notice that from our assumptions, we only have that the subgroup $G_{D}$ of $\left(\mathbb{Z}^{d},+\right)$ generated by the support of the law of $D$ is $d$-dimensional. The random walk $\left(T_{n}\right)$ lives in $G_{D}$. As $G_{D}$ admits a basis over $\mathbb{Z}$, a reparametrization of $G_{D}$ corresponds to making an injective linear change of variables in the integral in (4.2), with $\chi$ replaced by $\chi_{D}$. The properties of dominated variations shown in lemma 6.2 below imply that we can assume that $G_{D}=\mathbb{Z}^{d}$ from the beginning and this is what we do in the sequel.

The only singularity of $1 /\left(1-\chi_{D}\right)$ in $\mathbb{R}^{d} / \mathbb{Z}^{d}$ is now 0 . The symmetry coming from conjugation inside the real part in (4.2) implies that we can restrict the integral to the half unit ball $\left\{x \mid 0<\|x\| \leq \eta, x /\|x\| \in S_{+}^{d-1}\right\}$. Forgetting the multiplicative constant coming from the change of variables in polar coordinates, we decompose the integral in (4.2) in the form:

$$
\int_{\left.(u, t) \in S_{+}^{d-1} \times\right] 0, \eta[} \operatorname{Re}\left(\frac{1}{1-\chi_{D}(u t)}\right) t^{d-1} d u d t .
$$


Fixing $0<\eta<1 / 2$ small enough, we take $u \in S_{+}^{d-1}$ and $0<t<\eta$.

4.2. Local time and contour of a Galton-Watson tree. For $u \in S_{+}^{d-1}$ we shall study the behavior as $t \rightarrow 0^{+}$of $t \longmapsto \chi_{D}(u t)$. We require a description of $D$. In this direction, in order to detail the vertical component $\left(S_{n}^{2}\right)_{n \geq 0}$ of the random walk restricted to vertical jumps, introduce as in section 3.2 the one-dimensional Markov chain $\left(Y_{n}\right)_{n \geq 0}$ on $\mathbb{Z}$ with $Y_{0}=0$ and $\mathbb{P}_{n, n-1}=q_{n}^{\prime}, \mathbb{P}_{n, n+1}=p_{n}^{\prime}$, for $n \in \mathbb{Z}$. Let also $\sigma=\min \left\{k \geq 1 \mid Y_{k}=0\right\}$ be the return time to 0 of this random walk.

Grouping in packets the successive $\mathbb{Z}^{d}$-steps of the random walk, we observe that $D$ can be decomposed as:

$$
D=\sum_{k=0}^{\sigma-1}\left(\sum_{m=1}^{\Gamma_{k}} \xi_{m}^{(k)}\right) .
$$

Conditionally on the $\left(Y_{l}\right)_{l \geq 0}$, the $\left(\left(\xi_{m}^{(k)}\right)_{m \geq 1, k \geq 0},\left(\Gamma_{k}\right)_{k \geq 0}\right)$ are independent, with $\xi_{m}^{(k)} \sim \mu_{Y_{k}}$ and $\Gamma_{k} \sim\left(\mathcal{G}\left(r_{Y_{k}}\right)\right)$, for all $k \geq 0$. The level of complexity of the model of random walk under study is somehow condensed in the last formula.

Definition 4.3. Introduce for $n \in \mathbb{Z}$ the characteristic function:

$$
\varphi_{n}(u t)=\mathbb{E}\left(\exp \left(i t u . \sum_{m=1}^{\Gamma} \xi_{m}\right)\right), u \in S_{+}^{d-1}, t \in \mathbb{R},
$$

with random variables $\Gamma \sim \mathcal{G}\left(r_{n}\right)$ and $\xi_{m} \sim \mu_{n}$, for $m \geq 1$, being all independent.

Conditioning on the $\left(Y_{l}\right)_{l \geq 0}$ in (4.4), we obtain the equality:

$$
\chi_{D}(u t)=\mathbb{E}\left(\prod_{k=0}^{\sigma-1} \varphi_{Y_{k}}(u t)\right)=\varphi_{0}(u t) \mathbb{E}\left(\prod_{k=1}^{\sigma-1} \varphi_{Y_{k}}(u t)\right) .
$$

Observe that the only remaining alea is that of the $\left(Y_{l}\right)_{l \geq 0}$. Introduce the conditional expectations:

$$
\mathbb{E}^{+}(.)=\mathbb{E}\left(. \mid Y_{1}=1\right) \text { and } \mathbb{E}^{-}(.)=\mathbb{E}\left(. \mid Y_{1}=-1\right) .
$$

We set $\chi_{D}^{ \pm}(u t)=\mathbb{E}^{ \pm}\left(\prod_{k=1}^{\sigma-1} \varphi_{Y_{k}}(u t)\right)$. This leads to:

$$
\chi_{D}(u t)=\varphi_{0}(u t)\left(p_{0}^{\prime} \chi_{D}^{+}(u t)+q_{0}^{\prime} \chi_{D}^{-}(u t)\right) .
$$

We restrict the analysis to $\chi_{D}^{+}$, the case of $\chi_{D}^{-}$being symmetric. Introducing the local times $N_{n}=\#\left\{1 \leq k \leq \sigma-1, Y_{k}=n\right\}, n \geq 1$, of $\left(Y_{l}\right)$ on a positive excursion, we obtain:

$$
\chi_{D}^{+}(u t)=\mathbb{E}^{+}\left(\prod_{n \geq 1}\left(\varphi_{n}(u t)\right)^{N_{n}}\right) .
$$

The alea is now transferred on the $\left(N_{n}\right)_{n \geq 1}$. To describe these local times, one classically introduces (cf Le Gall, 2005 for instance) the Galton-Watson tree $\left(Z_{n}^{+}\right)_{n \geq 1}$ with $Z_{1}^{+}=1$ and such that, independently, the law of the number of children at level $n+1$ of an individual at level $n$ is $\mathcal{G}\left(p_{n}^{\prime}\right)$. This tree, introduced in section 3.2, is almost-surely finite, from the hypothesis $\lim _{n \rightarrow+\infty} v_{+}(n)=+\infty$. 


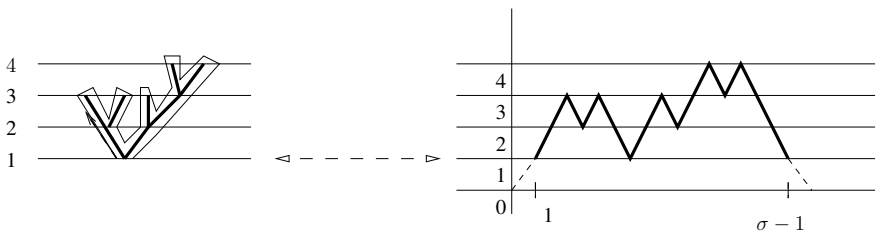

We next make the contour process of the tree, starting from the root and turning clockwise. This is the left-hand side of the picture. We associate to each ascending/descending movement $\mathrm{a}+1 /-1$ step. This gives the picture on the right-hand side, where we recover a positive excursion of the random walk $\left(Y_{l}\right)$ in the time interval $[1, \sigma-1]$. It is a simple observation that the total number of visits of the random walk at level $n \geq 1$ is $N_{n}=Z_{n}^{+}+Z_{n+1}^{+}$. This furnishes:

$$
\prod_{n \geq 1}\left(\varphi_{n}(u t)\right)^{N_{n}}=\prod_{n \geq 1}\left(\varphi_{n}(u t)\right)^{Z_{n}^{+}+Z_{n+1}^{+}}=\varphi_{1}(u t) \prod_{n \geq 1}\left[\varphi_{n}(u t) \varphi_{n+1}(u t)\right]^{Z_{n+1}^{+}}
$$

Finally we obtain the following formula for $\chi_{D}^{+}(u t)$ :

$$
\chi_{D}^{+}(u t)=\varphi_{1}(u t) \mathbb{E}^{+}\left(\prod_{n \geq 1}\left[\varphi_{n}(u t) \varphi_{n+1}(u t)\right]^{Z_{n+1}^{+}}\right)
$$

4.3. Development of $\chi_{D}^{+}$in SP-continued fraction. We now see that $\chi_{D}^{+}$can be naturally expressed as a SP-continued fraction. For $N \geq 1$, introduce the truncated version:

$$
\chi_{D}^{+, N}(u t)=\varphi_{1}(u t) \mathbb{E}^{+}\left(\prod_{n=1}^{N}\left[\varphi_{n}(u t) \varphi_{n+1}(u t)\right]^{Z_{n+1}^{+}}\right) .
$$

Observe that $\chi_{D}^{+, N}$ converges pointwise to $\chi_{D}^{+}$, by dominated convergence inside the expectation.

We now use the classical description of the Galton-Watson tree $\left(Z_{n}^{+}\right)_{n \geq 1}$. Let $\left(R_{k, n}\right)_{n \geq 1, k \geq 1}$ be independent random variables such that $R_{k, n} \sim \mathcal{G}\left(p_{n}^{\prime}\right)$. Then we have:

$$
Z_{1}^{+}=1, Z_{n+1}^{+}=\sum_{k=1}^{Z_{n}^{+}} R_{k, n}, n \geq 1
$$

Recall that the generating function of $\mathcal{G}\left(p_{n}^{\prime}\right)$ is $s \longmapsto q_{n}^{\prime} /\left(1-p_{n}^{\prime} s\right)=a_{n} /\left(b_{n}-s\right)$, $0 \leq s \leq 1$. Conditioning in the middle step below, this allows to write:

$$
\begin{aligned}
\chi_{D}^{+, N}(u t) & =\varphi_{1}(u t) \mathbb{E}^{+}\left(\prod_{n=1}^{N-1}\left[\varphi_{n}(u t) \varphi_{n+1}(u t)\right]^{Z_{n+1}^{+}\left(\varphi_{N}(u t) \varphi_{N+1}(u t)\right)^{Z_{N+1}^{+}}}\right) \\
& =\varphi_{1}(u t) \mathbb{E}^{+}\left(\prod_{n=1}^{N-1}\left[\varphi_{n}(u t) \varphi_{n+1}(u t)\right]^{Z_{n+1}^{+}}\left(\frac{a_{N}}{b_{N}-\varphi_{N}(u t) \varphi_{N+1}(u t)}\right)^{Z_{N}^{+}}\right) \\
& =\varphi_{1}(u t) \mathbb{E}^{+}\left(\prod_{n=1}^{N-2}\left[\varphi_{n}(u t) \varphi_{n+1}(u t)\right]^{Z_{n+1}^{+}}\left(\frac{a_{N} \varphi_{N-1}(u t) \varphi_{N}(u t)}{b_{N}-\varphi_{N}(u t) \varphi_{N+1}(u t)}\right)^{Z_{N}^{+}}\right) .
\end{aligned}
$$


Iterating the procedure, replacing $\varphi_{N} \varphi_{N+1}$ of the first line by $\frac{a_{N} \varphi_{N-1} \varphi_{N}}{b_{N}-\varphi_{N} \varphi_{N+1}}$, we obtain:

$$
\chi_{D}^{+, N}=\frac{\varphi_{1} a_{1}}{b_{1}-\frac{\varphi_{1} \varphi_{2} a_{2}}{b_{2} \cdots-\frac{\varphi_{N-1} \varphi_{N} a_{N}}{b_{N}-\varphi_{N} \varphi_{N+1}}}} .
$$

Notice that the $\varphi_{n}$ are close to 1 , hence not 0 , uniformly in $n$ and $u \in S_{+}^{d-1}$ for small $t$. This follows from hypothesis 1.1, giving a second order expansion of $\varphi_{k}(u t)$ with uniform remainder term (see the beginning of the next section for more details). Dividing above by $\varphi_{1}, \cdots, \varphi_{N}$ at each successive level, we get:

$\chi_{D}^{+, N}(u t)=\left[\left(a_{1}, b_{1} / \varphi_{1}(u t)\right) ;\left(-a_{2}, b_{2} / \varphi_{2}(u t)\right) ; \cdots ;\left(-a_{N}, b_{N} / \varphi_{N}(u t)-\varphi_{N+1}(u t)\right)\right]$.

From the pointwise convergence of $\chi_{D}^{+, N}$ and lemma 3.1, we conclude that:

$$
\chi_{D}^{+}(u t)=\left[\left(a_{1}, b_{1} / \varphi_{1}(u t)\right) ;\left(-a_{2}, b_{2} / \varphi_{2}(u t)\right) ; \cdots ;\left(-a_{n}, b_{n} / \varphi_{n}(u t)\right) \cdots\right] .
$$

Naturally, a similar expression is true for $\chi_{D}^{-}(u t)$. We may observe that we have in fact established something slightly stronger:

Lemma 4.4. Let $\left(\gamma_{n}\right)_{n \geq 1}$ be a sequence of complex numbers with $0<\left|\gamma_{n}\right| \leq 1$, $n \geq 1$. Then:

$$
\begin{aligned}
\mathbb{E}^{+} \prod_{k=1}^{\sigma-1} \gamma_{Y_{k}} & =\gamma_{1} \mathbb{E}^{+} \prod_{n \geq 1}\left[\gamma_{n} \gamma_{n+1}\right]^{Z_{n+1}^{+}} \\
& =\left[\left(a_{1}, b_{1} / \gamma_{1}\right) ;\left(-a_{2}, b_{2} / \gamma_{2}\right) ; \cdots ;\left(-a_{n}, b_{n} / \gamma_{n}\right) ; \cdots\right]
\end{aligned}
$$

This is shown by simply replacing $\left(\varphi_{n}(u t)\right)_{n \geq 1}$ by $\left(\gamma_{n}\right)_{n \geq 1}$ in the previous proof.

4.4. Another reduction. Let $\hat{\mu}_{n}(u t)=\sum_{k \in \mathbb{Z}^{d}} e^{i t u . k} \mu_{n}(k), u \in S_{+}^{d-1}, t \in \mathbb{R}$. From (4.5), we obtain:

$$
\varphi_{n}(u t)=\left(1-r_{n}\right) /\left(1-r_{n} \hat{\mu}_{n}(u t)\right) .
$$

As a result, we have the following expansion:

$$
\frac{1}{\varphi_{n}(u t)}=1+i t u . m_{n} \frac{r_{n}}{1-r_{n}}+O\left(t^{2}\right),
$$

with $O$ uniform in $n$ and $u \in S_{+}^{d-1}$.

\section{Definition 4.5.}

1) For $n \in \mathbb{Z}$, let $\eta_{n}=r_{n} m_{n} / p_{n}$.

2) For $n \in \mathbb{Z}, u \in S_{+}^{d-1}$ and $t \in \mathbb{R}$ small enough (uniformly in $n$ and $u$, by hypothesis 1.1), set:

$$
\frac{1}{\psi_{n}(u t)}=1+i t u . \eta_{n} \frac{p_{n}}{1-r_{n}}=1+i t u . \eta_{n} / b_{n} .
$$

Our aim is to replace below the $\varphi_{n}(u t)$ by the $\psi_{n}(u t)$ in the recursive relation (3.4) satisfied by the denominators $\left(B_{n}\right)$ of the partial quotients of the SP-continued fraction expansion of $\chi_{D}^{+}(u t)$.

Lemma 4.6. Let $c=\delta^{3} / 4>0$ (where $\delta$ comes from hypothesis 1.1). For small $t>0$, uniformly in $n$ and $u \in S_{+}^{d-1}$, we have the inequality:

$$
\left|\varphi_{n}(u t)\right| \leq 1-c t^{2}
$$


Proof: Let $M_{2, n}(u)=\sum_{k \in \mathbb{Z}^{d}}(k . u)^{2} \mu_{n}(k), m_{n}(u)=m_{n} . u$ and $\operatorname{Var}_{n}(u)=$ $M_{2, n}(u)-\left(m_{n}(u)\right)^{2}$. A computation gives:

$$
\left|\varphi_{n}(u t)\right|=1-\frac{t^{2}}{2} \frac{r_{n}}{\left(1-r_{n}\right)^{2}}\left(M_{2, n}(u)-r_{n} \operatorname{Var}_{n}(u)\right)+O\left(t^{3}\right),
$$

with $O$ uniform in $n$ and $u \in S^{d-1}$, due to the uniformly bounded third moment of $\mu_{n}$. Using the hypotheses, we have $\delta^{2} \leq \delta M_{2, n}(u) \leq M_{2, n}(u)-r_{n} \operatorname{Var}_{n}(u)$. Hence:

$$
\left|\varphi_{n}(u t)\right| \leq 1-\frac{t^{2} \delta^{3}}{2}+O\left(t^{3}\right) \leq 1-\frac{t^{2} \delta^{3}}{4},
$$

for $t$ small enough, uniformly in $n$ and $u \in S_{+}^{d-1}$.

Let us now introduce some definitions.

\section{Definition 4.7.}

1) Introduce for $n \geq 0$ :

$$
w_{+}(n)=\sum_{0 \leq k \leq n}\left(1 / \rho_{k}\right) \text { and } w_{-}(n)=\left(1 / a_{0}\right) \sum_{-n-1 \leq k \leq-1}\left(1 / \rho_{k}\right) .
$$

2) Let $F_{+}(n)=\left(n w_{+} \circ v_{+}^{-1}(n)\right)^{1 / 2}, n \geq 0$.

3) Let $R^{+}(t)=1-\mathbb{E}^{+}\left(\left(1-t^{2}\right)^{\sigma-1}\right)$ and $f^{+}(u t)=\mathbb{E}^{+}\left(\prod_{k=1}^{\sigma-1} \psi_{Y_{k}}(u t)\right)$.

The main result of this section is the following.

\section{Lemma 4.8.}

1) For $C \geq 1$ and large enough $x>0: F_{+}^{-1}(C x) \leq 2 C^{2} F_{+}^{-1}(x)$.

2) There exists $\alpha \geq 1$ so that for small $t>0$ :

$$
\frac{1}{\alpha} \leq R^{+}(t) F_{+}^{-1}(1 / t) \leq \alpha .
$$

3) There exist constants $C_{1}>0, C_{2}>0$ so that for small $t>0$, uniformly in $u \in S_{+}^{d-1}$ :

$$
1-\left|\chi_{D}^{+}(u t)\right| \geq C_{1} R^{+}(t) \text { and }\left|\chi_{D}^{+}(u t)-f^{+}(u t)\right| \leq C_{2} R^{+}(t) .
$$

Proof: 1) Note that $F_{+}(n) \rightarrow+\infty$, as $n \rightarrow+\infty$ and that $n \longmapsto F_{+}^{2}(n) / n$ is nondecreasing. Let $C \geq 1$ and $x>0$. Set $n=F_{+}^{-1}(x)$ and suppose that $n \geq 1$. By definition, $F_{+}(n) \leq x<F_{+}(n+1)$. Similarly, let $n+p=F_{+}^{-1}(C x)$. Then:

$$
\frac{F_{+}^{-1}(C x)}{F_{+}^{-1}(x)}=\frac{n+p}{n} \leq 2 \frac{n+p}{n+1} \leq 2 \frac{F_{+}^{2}(n+p)}{F_{+}^{2}(n+1)} \leq 2 \frac{C^{2} x^{2}}{x^{2}}=2 C^{2} .
$$

This completes the proof of the assertion.

As a preliminary remark for what follows, set now, for $n \geq 1, \Theta_{+}(n)=$ $\left(\sum_{1 \leq k \leq l \leq n}\left(\rho_{l} / \rho_{k}\right)\right)^{1 / 2}$. Let $c>0$ be such that for all $k \geq 1$ :

$$
w_{+}(k) \leq c \sum_{1 \leq u \leq k}\left(1 / \rho_{u}\right), v_{+}(k+1) \leq c v_{+}(k) \text { and } \Theta_{+}(k+1) \leq c \Theta_{+}(k) .
$$

We claim that there exists $C \geq 1$ so that for all $x>0$ large enough:

$$
(1 / C) v_{+} \circ \Theta_{+}^{-1}(x) \leq F_{+}^{-1}(x) \leq C v_{+} \circ \Theta_{+}^{-1}(x) .
$$


For $x>0$, let $n=\Theta_{+}^{-1}(x)$, so that $\Theta_{+}(n) \leq x<\Theta_{+}(n+1)$. We have, using that $n=v_{+}^{-1}\left(v_{+}(n)\right)$ and denoting the by $\lceil y\rceil$ the smallest integer $\geq y$ :

$$
\begin{aligned}
\frac{x^{2}}{c^{2}} & \leq \Theta_{+}^{2}(n) \leq v_{+}(n) \sum_{0 \leq l \leq n}\left(1 / \rho_{l}\right) \\
& \leq c v_{+}(n) w_{+}(n) \leq c\left\lceil v_{+}(n)\right\rceil w_{+} \circ v_{+}^{-1}\left(\left\lceil v_{+}(n)\right\rceil\right)=c F_{+}^{2}\left(\left\lceil v_{+}(n)\right\rceil\right) .
\end{aligned}
$$

As a result, for large $n, v_{+}(n) \geq\left\lceil v_{+}(n)\right\rceil / 2 \geq F_{+}^{-1}\left(x c^{-3 / 2}\right) / 2 \geq K F_{+}^{-1}(x)$, by the first point. On the other hand, let $m=v_{+}^{-1}\left(v_{+}(n) / 2\right)$. We have $v_{+}(m) \leq$ $v_{+}(n) / 2<v_{+}(m+1)$. This gives:

$$
\begin{aligned}
x^{2} \geq \Theta_{+}^{2}(n) & \geq \sum_{1 \leq k \leq m}\left(1 / \rho_{k}\right) \sum_{m \leq l \leq n} \rho_{l} \\
& \geq \frac{1}{c} w_{+}(m)\left(v_{+}(n)-v_{+}(m)\right) \geq \frac{1}{c} F_{+}^{2}\left(\left\lfloor v_{+}(n) / 2\right\rfloor\right),
\end{aligned}
$$

introducing the integer part $\lfloor y\rfloor$. To conclude, for $n$ large enough, $v_{+}(n) \leq$ $3\left\lfloor v_{+}(n) / 2\right\rfloor \leq 3 F_{+}^{-1}\left(x c^{1 / 2}\right) \leq K^{\prime} F_{+}^{-1}(x)$, by the first point.

2) Let us turn to the evaluation of $R^{+}(t)$. Using lemmas 4.4 and 3.1 we have:

$$
\mathbb{E}^{+}\left(\left(1-t^{2}\right)^{\sigma-1}\right)=\lim _{n \rightarrow+\infty} \frac{\alpha_{n}(t)}{\beta_{n}(t)}=\sum_{n \geq 1} \frac{\rho_{n}}{\beta_{n} \beta_{n-1}},
$$

where $\beta_{-1}=0, \beta_{0}=1, \beta_{1}=b_{1} /\left(1-t^{2}\right)$ and $\beta_{n}=\left(b_{n} /\left(1-t^{2}\right)\right) \beta_{n-1}-a_{n} \beta_{n-2}, n \geq 2$. We omit the dependence in $t$. The $\left(\alpha_{n}\right)$ satisfy the same recursive relation with this time $\alpha_{-1}=1, \alpha_{0}=0$ and $\alpha_{1}=a_{1}$. First of all, as $\Theta_{+}(n) \rightarrow+\infty$, as $n \rightarrow+\infty$, and there is a constant $C>0$ so that for all $n \geq 1, \Theta_{+}(n) \leq \Theta_{+}(n+1) \leq C \Theta_{+}(n)$, we deduce that for any constant $c>0$ (chosen later), there exists a constant $c^{\prime}>0$ so that for small enough $t>0$ there is an integer $N(t)$ so that:

$$
\frac{c^{\prime}}{t^{2}} \leq \Theta_{+}^{2}(N(t)) \leq \frac{c}{t^{2}}
$$

This next furnishes, using lemma 3.1:

$$
\left|R^{+}(t)-\left(\frac{\beta_{N(t)}(t)-\alpha_{N(t)}(t)}{\beta_{N(t)}(t)}\right)\right| \leq \sum_{n>N(t)} \frac{\rho_{n}}{\beta_{n}(t) \beta_{n-1}(t)} \leq \frac{1}{\beta_{N(t)}} .
$$

We shall show that there exists a constant $\varepsilon>0$ so that $1+\varepsilon \leq \beta_{N(t)}(t)-\alpha_{N(t)}(t) \leq$ $1 / \varepsilon$ and next that $v_{+}(N(t)) \leq \beta_{N(t)}(t) \leq v_{+}(N(t)) / \varepsilon$. These two properties imply that $R^{+}(t)$ has exact order $1 / v_{+}(N(t))$ and so $1 / F_{+}^{-1}(1 / t)$, by the first point.

We have $b_{n} /\left(1-t^{2}\right)=b_{n}+t^{2} c_{n}(t)$, with $(1 / \alpha) \leq c_{n}(t) \leq \alpha$, for some constant $\alpha>0$. Next:

$$
\left(\begin{array}{c}
\beta_{n} \\
\beta_{n-1}
\end{array}\right)=\left(\begin{array}{cc}
b_{n}+t^{2} c_{n}(t) & -a_{n} \\
1 & 0
\end{array}\right) \cdots\left(\begin{array}{cc}
b_{1}+t^{2} c_{1}(t) & -a_{1} \\
1 & 0
\end{array}\right)\left(\begin{array}{l}
1 \\
0
\end{array}\right), n \geq 0 .
$$

Introduce now $C_{n}=\left(\begin{array}{cc}b_{n} & -a_{n} \\ 1 & 0\end{array}\right), B=\left(\begin{array}{ll}1 & 0 \\ 0 & 0\end{array}\right)$. Since $\beta_{n}(0)=v_{+}(n)$ and for $k, l \geq 0$ we have $\left\langle e_{1}, C_{k+l} \cdots C_{k+1} e_{1}\right\rangle=\theta^{k} v_{+}(l)$, when developing the product we 
obtain for $n \geq 0$ :

$$
\begin{aligned}
& \beta_{n}= \\
& =v_{+}(n)+\sum_{r=1}^{n} t^{2 r} \sum_{1 \leq k_{1}<\cdots<k_{r} \leq n} c_{k_{1}}(t) \cdots c_{k_{r}}(t)\left\langle e_{1}, C_{n} \cdots C_{k_{r}+1} B \cdots B C_{k_{1}-1} \cdots C_{1} e_{1}\right\rangle \\
& =v_{+}(n)+\sum_{r=1}^{n} t^{2 r} \sum_{1 \leq k_{1}<\cdots<k_{r} \leq n} c_{k_{1}}(t) \cdots c_{k_{r}}(t)\left\langle e_{1}, C_{n} \cdots C_{k_{r}+1} e_{1}\right\rangle \cdots\left\langle e_{1}, C_{k_{1}-1} \cdots C_{1} e_{1}\right\rangle \\
& =v_{+}(n) \\
& +\sum_{r=1}^{n} t^{2 r} \sum_{1 \leq k_{1}<\cdots<k_{r} \leq n}\left(c_{k_{1}} \cdots c_{k_{r}}\right)(t) v_{+}\left(k_{1}-1\right) \theta^{k_{1}} v_{+}\left(k_{2}-k_{1}-1\right) \cdots \theta^{k_{r}} v_{+}\left(n-k_{r}\right) .
\end{aligned}
$$

Idem, since $\alpha_{n}(0)=v_{+}(n)-1$ and $\alpha_{n}=a_{1} \theta \beta_{n-1}$, for $n \geq 0$, as follows from the recursive relation:

$$
\begin{aligned}
& \alpha_{n}=v_{+}(n)-1 \\
& +\sum_{r=1}^{n} t^{2 r} \sum_{2 \leq k_{1}<\cdots<k_{r} \leq n}\left(c_{k_{1}} \cdots c_{k_{r}}\right)(t)\left(v_{+}\left(k_{1}-1\right)-1\right) \theta^{k_{1}} v_{+}\left(k_{2}-k_{1}-1\right) \cdots \theta^{k_{r}} v_{+}\left(n-k_{r}\right) .
\end{aligned}
$$

This furnishes:

$$
\beta_{n}-\alpha_{n}=1+\sum_{r=1}^{n} t^{2 r} \sum_{1 \leq k_{1}<\cdots<k_{r} \leq n}\left(c_{k_{1}} \cdots c_{k_{r}}\right)(t) \theta^{k_{1}} v_{+}\left(k_{2}-k_{1}-1\right) \cdots \theta^{k_{r}} v_{+}\left(n-k_{r}\right) \text {. }
$$

As a result, the equality for $\beta_{n}$ gives $\beta_{n} \leq v_{+}(n)\left(1+\sum_{1 \leq r \leq n} \alpha^{r} t^{2 r}\left(\Theta_{+}^{2}(n)\right)^{r}\right)$. Also, by the above, $\beta_{n}-\alpha_{n} \geq 1+t^{2} \Theta_{+}^{2}(n) / \alpha$, because:

$$
\sum_{1 \leq k \leq n} \theta_{k} v_{+}(n-k)=\Theta_{+}(n)
$$

According to the previous discussion, we simply choose $0<c \leq \alpha / 2$ to get the desired result.

3) We have $\chi_{D}^{+}(u t)=\mathbb{E}^{+}\left(\prod_{k=1}^{\sigma-1} \varphi_{Y_{k}}(u t)\right)$. By $(4.8),\left|\chi_{D}^{+}(u t)\right| \leq \mathbb{E}^{+}\left(\left(1-c t^{2}\right)^{\sigma-1}\right)=$ $1-R^{+}(\sqrt{c} t)$. This gives the first inequality, as the first point of the lemma says that $R^{+}(\sqrt{c} t) \leq C R^{+}(t)$, for some constant $C$ depending on $c$. Concerning the second inequality:

$$
\begin{aligned}
\left|\chi_{D}^{+}(u t)-f^{+}(u t)\right| & =\left|\mathbb{E}^{+}\left(\prod_{k=1}^{\sigma-1} \varphi_{Y_{k}}(u t)\right)-\mathbb{E}^{+}\left(\prod_{k=1}^{\sigma-1} \psi_{Y_{k}}(u t)\right)\right| \\
& =\left|\mathbb{E}^{+}\left(\sum_{k=1}^{\sigma-1}\left(\prod_{l=1}^{k-1} \varphi_{Y_{l}}(u t)\left(\varphi_{Y_{k}}(u t)-\psi_{Y_{k}}(u t)\right) \prod_{l=k+1}^{\sigma-1} \psi_{Y_{l}}(u t)\right)\right)\right| . \\
& \leq \mathbb{E}^{+}\left(\sum_{k=1}^{\sigma-1}\left(\prod_{l=1}^{k-1}\left|\varphi_{Y_{l}}(u t)\right|\left|\varphi_{Y_{k}}(u t)-\psi_{Y_{k}}(u t)\right| \prod_{l=k+1}^{\sigma-1}\left|\psi_{Y_{l}}(u t)\right|\right)\right) .
\end{aligned}
$$

Using now that for some $C>0$ and small enough $t>0$, uniformly in $n$ and $u \in S_{+}^{d-1},\left|\varphi_{n}(u t)-\psi_{n}(u t)\right| \leq C t^{2}$, as well as $\left|\varphi_{n}(u t)\right| \leq 1-c t^{2}$ and $\left|\psi_{n}(u t)\right| \leq 1$, 
we get for small $t>0$ :

$$
\begin{aligned}
\left|\chi_{D}^{+}(u t)-f^{+}(u t)\right| & \leq C t^{2} \mathbb{E}^{+}\left(\sum_{k=1}^{\sigma-1}\left(1-c t^{2}\right)^{k-1}\right) \\
& =C t^{2} \mathbb{E}^{+}\left(\frac{1-\left(1-c t^{2}\right)^{\sigma-1}}{c t^{2}}\right)=\frac{C}{c} R^{+}(\sqrt{c} t) .
\end{aligned}
$$

The conclusion now comes from the first point of the lemma.

\section{Precise analysis of some convergents}

A summary of the previous section is that as $t \rightarrow 0^{+}$, uniformly in $u \in S_{+}^{d-1}$ :

$$
\chi_{D}^{+}(u t)=f^{+}(u t)+O\left(R^{+}(t)\right)
$$

with $f^{+}(u t)=\lim _{n \rightarrow+\infty} A_{n}(u t) / B_{n}(u t)$, where now:

$$
\left(\begin{array}{c}
B_{n}(u t) \\
B_{n-1}(u t)
\end{array}\right)=\left(\begin{array}{cc}
b_{n}-i t u . \eta_{n} & -a_{n} \\
1 & 0
\end{array}\right) \cdots\left(\begin{array}{cc}
b_{1}-i t u . \eta_{1} & -a_{1} \\
1 & 0
\end{array}\right)\left(\begin{array}{l}
1 \\
0
\end{array}\right), n \geq 0
$$

together with $A_{n}(u t)=a_{1} \theta B_{n-1}(u t), n \geq 0$. The aim of the present section is to study in detail $\left(A_{n}(u t)\right)$ and $\left(B_{n}(u t)\right)$. Let us introduce the following definitions.

Definition 5.1. Let $u \in S_{+}^{d-1}$. For $k \in \mathbb{Z}$, let $\eta_{k}^{u}=\eta_{k}$. $u$. For $k \leq l$ in $\mathbb{Z}$, set:

$$
R_{k}^{l}(u)=\sum_{k \leq r \leq l} \eta_{r}^{u}\left(\rho_{l} / \rho_{r}\right) \text { and } T_{k}^{l}(u)=\left(R_{k}^{l}(u)\right)^{2} \rho_{k-1} / \rho_{l} .
$$

These quantities depend only in the data in $[k, l]$. For $0 \leq r \leq n$, introduce also:

$$
\Delta_{r}^{n}(u)=\sum_{1 \leq k_{1}<\cdots<k_{r} \leq n} R_{1}^{k_{1}}(u) R_{k_{1}+1}^{k_{2}}(u) \cdots R_{k_{r-1}+1}^{k_{r}}(u)
$$

with the convention that $\Delta_{0}^{n}(u)=1$ and $\Delta_{r}^{n}(u)=0$ if $r>n$ or $r<0$.

We first proceed exactly as in the proof of the second point of lemma 4.8. Fixing $u \in S_{+}^{d-1}$, we develop (5.1) and obtain for $n \geq 0$ :

$$
\begin{aligned}
& B_{n}(u t)=v_{+}(n) \\
& +\sum_{r=1}^{n}(-i t)^{r} \sum_{\substack{1 \leq k_{1}<\cdots<k_{r} \leq n \\
k_{1}}}^{u} \cdots \eta_{k_{r}}^{u} v_{+}\left(k_{1}-1\right) \theta^{k_{1}} v_{+}\left(k_{2}-k_{1}-1\right) \cdots \theta^{k_{r}} v_{+}\left(n-k_{r}\right), \\
& A_{n}(u t)=v_{+}(n)-1 \\
& +\sum_{r=1}^{n}(-i t)^{r} \sum_{2 \leq k_{1}<\cdots<k_{r} \leq n} \eta_{k_{1}}^{u} \cdots \eta_{k_{r}}^{u}\left(v_{+}\left(k_{1}-1\right)-1\right) \theta^{k_{1}} v_{+}\left(k_{2}-k_{1}-1\right) \cdots \theta^{k_{r}} v_{+}\left(n-k_{r}\right) .
\end{aligned}
$$

We therefore deduce the equality:

$$
B_{n}(u t)-A_{n}(u t)=1+\sum_{r=1}^{n}(-i t)^{r} \sum_{1 \leq k_{1}<\cdots<k_{r} \leq n} \eta_{k_{1}}^{u} \cdots \eta_{k_{r}}^{u} \theta^{k_{1}} v_{+}\left(k_{2}-k_{1}-1\right) \cdots \theta^{k_{r}} v_{+}\left(n-k_{r}\right) .
$$


Let us consider the last sum. We first fix $k_{2}, \cdots, k_{r}$ and write:

$$
\begin{aligned}
\sum_{1 \leq k_{1}<k_{2}} \eta_{k_{1}}^{u} \theta^{k_{1}} v_{+}\left(k_{2}-k_{1}-1\right) & =\sum_{1 \leq k_{1}<k_{2}} \eta_{k_{1}}^{u} \sum_{k_{1} \leq l<k_{2}} \frac{\rho_{l}}{\rho_{k_{1}}} \\
& =\sum_{1 \leq l<k_{2}} \sum_{1 \leq k_{1} \leq l} \eta_{k_{1}}^{u} \frac{\rho_{l}}{\rho_{k_{1}}}=\sum_{1 \leq l<k_{2}} R_{1}^{l}(u) .
\end{aligned}
$$

Successively iterate this manipulation for $k_{2}, \cdots, k_{r}$ in the formula for $B_{n}(u t)-$ $A_{n}(u t)$. Then:

$$
\begin{aligned}
B_{n}(u t)-A_{n}(u t) & =1+\sum_{r=1}^{n}(-i t)^{r} \sum_{1 \leq k_{1}<\cdots<k_{r} \leq n} R_{1}^{k_{1}}(u) R_{k_{1}+1}^{k_{2}}(u) \cdots R_{k_{r-1}+1}^{k_{r}}(u) \\
& =\sum_{r=0}^{n}(-i t)^{r} \Delta_{r}^{n}(u) .
\end{aligned}
$$

Similarly, using as first step that $\sum_{1 \leq k_{1}<k_{2}} \eta_{k_{1}}^{u} v_{+}\left(k_{1}-1\right) \theta^{k_{1}} v_{+}\left(k_{2}-k_{1}-1\right)=$ $\sum_{0 \leq s<l<k_{2}} \rho_{s} R_{s+1}^{l}(u)$ :

$$
\begin{aligned}
B_{n}(u t) & =v_{+}(n)+\sum_{r=1}^{n}(-i t)^{r} \sum_{0 \leq k_{1}<\cdots<k_{r+1} \leq n} \rho_{k_{1}} R_{k_{1}+1}^{k_{2}}(u) \cdots R_{k_{r}+1}^{k_{r+1}}(u) \\
& =\sum_{r=0}^{n}(-i t)^{r} \sum_{0 \leq k \leq n} \rho_{k} \theta^{k} \Delta_{r}^{n-k}(u) .
\end{aligned}
$$

Proposition 5.2. Set $2^{(k, l)}=2$ if $k \neq l$ and 1 if $k=l$. We have the following exact computations:

(1) $\left|B_{n}(u t)-A_{n}(u t)\right|^{2}=\sum_{r=0}^{n} t^{2 r} K_{r}^{u}(n)$, with:

$$
K_{r}^{u}(n)=\sum_{1 \leq l_{1}<k_{2} \leq l_{2}<\cdots<k_{r} \leq l_{r}<k_{r+1} \leq n+1} T_{1}^{l_{1}}(u) \cdots T_{k_{r}}^{l_{r}}(u) \rho_{k_{r+1}-1} 2^{H_{r}\left(\left(k_{i}\right),\left(l_{j}\right)\right)}
$$

where $H_{r}\left(\left(k_{i}\right),\left(l_{j}\right)\right):=\#\left\{1 \leq i \leq r \mid l_{i}+1<k_{i+1}\right\}$.

(2) $\left|B_{n}(u t)\right|^{2}=\sum_{r=0}^{n} t^{2 r} L_{r}^{u}(n)$, with:

$$
L_{r}^{u}(n)=\sum_{0 \leq k \leq l \leq n} \rho_{k} \rho_{l} 2^{(k, l)} \theta^{l} K_{r}^{u}(n-l) .
$$

(3) $\operatorname{Re}\left(\left(B_{n}-A_{n}\right) \bar{B}_{n}\right)(u t)=\sum_{r=0}^{n} t^{2 r} M_{r}^{u}(n)$, with:

$$
M_{r}^{u}(n)=\sum_{0 \leq k \leq n} \rho_{k} \theta^{k} K_{r}^{u}(n-k) .
$$

(4) $\operatorname{Im}\left(A_{n}(u t) \bar{B}_{n}(u t)\right)=\sum_{r=0}^{n-1} t^{2 r+1} N_{r}^{u}(n)$, with:

$$
N_{r}^{u}(n)=\sum_{1 \leq k \leq l \leq n} R_{1}^{k} 2^{(k, l)} \rho_{l} \theta^{l} K_{r}^{u}(n-l) .
$$

When $r>n$ or $r<0$, set $K_{r}^{u}(n)=L_{r}^{u}(n)=M_{r}^{u}(n)=0$. Idem $N_{r}^{u}(n)=0, r \geq n$ or $r<0$.

Proof: In the following, we suppress the dependence in $u$ of $R_{k}^{l}, T_{k}^{l}, K_{r}(n), L_{r}(n)$, $M_{r}(n), N_{r}(n)$ and $\Delta_{r}^{n}$ to slightly lighten the notations. 
1. Since $B_{n}(u t)-A_{n}(u t)=\sum_{0 \leq r \leq n}(-i t)^{r} \Delta_{r}^{n}$, this gives:

$$
\begin{aligned}
\left|B_{n}(u t)-A_{n}(u t)\right|^{2} & =\left(B_{n}(u t)-A_{n}(u t) \overline{\left(B_{n}(u t)-A_{n}(u t)\right)}\right. \\
& =\sum_{r=0}^{n} t^{2 r} \sum_{p=-r}^{r} \Delta_{r+p}^{n} \Delta_{r-p}^{n}(-i)^{r+p} i^{r-p},
\end{aligned}
$$

using the conventions for $\Delta_{r}^{n}$ concerning the value of $r$ with respect to $n$. Hence $\left|B_{n}(u t)-A_{n}(u t)\right|^{2}=\sum_{r=0}^{n} t^{2 r} K_{r}(n)$, with $K_{0}(n)=1$ and:

$$
K_{r}(n)=\sum_{p=-r}^{r}(-1)^{p} \Delta_{r+p}^{n} \Delta_{r-p}^{n}, r \geq 1 .
$$

We will show that the following recursive relations are verified:

$$
\begin{aligned}
K_{1}(n) & =\sum_{1 \leq k \leq l \leq n} T_{1}^{k} \rho_{l} 2^{(k, l)}, \\
K_{r}(n) & =\sum_{1 \leq k \leq l \leq n} T_{1}^{k} \rho_{l} \theta^{l} K_{r-1}(n-l) 2^{(k, l)}, \text { for } r \geq 2 .
\end{aligned}
$$

This then gives the announced formula.

For the initial relation:

$$
\begin{aligned}
K_{1}(n) & =\left(\Delta_{1}^{n}\right)^{2}-2 \Delta_{2}^{n}=\left(\sum_{1 \leq k \leq n} R_{1}^{k}\right)^{2}-2 \sum_{1 \leq k<l \leq n} R_{1}^{k} R_{k+1}^{l} \\
& =\sum_{1 \leq k \leq n}\left(R_{1}^{k}\right)^{2}+2 \sum_{1 \leq k<l \leq n} R_{1}^{k}\left(R_{1}^{l}-R_{k+1}^{l}\right) .
\end{aligned}
$$

Observing that $R_{1}^{k}\left(R_{1}^{l}-R_{k+1}^{l}\right)=\left(R_{1}^{k}\right)^{2}\left(\rho_{l} / \rho_{k}\right)=T_{1}^{k} \rho_{l}$, this proves (5.2).

Let us now turn to the proof of (5.3). Taking first general $p \geq 1$ and $q \geq 1$, we write:

$$
\Delta_{p}^{n} \Delta_{q}^{n}=\sum_{\substack{1 \leq k_{1}<\cdots<k_{p} \leq n \\ 1 \leq k_{1}^{\prime}<\cdots<k_{q}^{\prime} \leq n}}\left(R_{1}^{k_{1}} \cdots R_{k_{p-1}+1}^{k_{p}}\right)\left(R_{1}^{k_{1}^{\prime}} \cdots R_{k_{q-1}^{\prime}+1}^{k_{q}^{\prime}}\right) .
$$

Distinguishing the cases $k_{1}=k_{1}^{\prime}, k_{1}<k_{1}^{\prime}$ and $k_{1}^{\prime}<k_{1}$, we decompose:

$$
\begin{aligned}
& \Delta_{p}^{n} \Delta_{q}^{n}=\sum_{1 \leq k \leq n}\left(R_{1}^{k}\right)^{2} \theta^{k} \Delta_{p-1}^{n-k} \theta^{k} \Delta_{q-1}^{n-k} \\
& +\sum_{\substack{1 \leq k_{1}<\cdots<k_{p} \leq n \\
k_{1}<k_{1}^{\prime}<\cdots<k_{q}^{\prime} \leq n}} R_{1}^{k_{1}}\left(R_{k_{1}+1}^{k_{2}} \cdots R_{k_{p-1}+1}^{k_{p}}\right)\left(R_{1}^{k_{1}} \frac{\rho_{k_{1}^{\prime}}}{\rho_{k_{1}}}+R_{k_{1}+1}^{k_{1}^{\prime}}\right)\left(R_{k_{1}^{\prime}+1}^{k_{2}^{\prime}} \cdots R_{k_{q-1}^{\prime}+1}^{k_{q}^{\prime}}\right) \\
& +\sum_{\substack{1 \leq k_{1}^{\prime}<\cdots<k_{q}^{\prime} \leq n \\
k_{1}^{\prime}<k_{1}<\cdots<k_{p} \leq n}}\left(R_{1}^{k_{1}^{\prime}} \frac{\rho_{k_{1}}}{\rho_{k_{1}^{\prime}}}+R_{k_{1}^{\prime}+1}^{k_{1}}\right)\left(R_{k_{1}+1}^{k_{2}} \cdots R_{k_{p-1}+1}^{k_{p}}\right)\left(R_{1}^{k_{1}^{\prime}} \cdots R_{k_{q-1}^{\prime}+1}^{k_{q}^{\prime}}\right) .
\end{aligned}
$$

Regrouping terms, this is rewritten as:

$$
\begin{aligned}
\Delta_{p}^{n} \Delta_{q}^{n} & =\sum_{1 \leq k \leq n}\left(R_{1}^{k}\right)^{2}\left[\theta^{k} \Delta_{p-1}^{n-k} \sum_{k \leq l \leq n} \theta^{l} \Delta_{q-1}^{n-l} \frac{\rho_{l}}{\rho_{k}}+\theta^{k} \Delta_{q-1}^{n-k} \sum_{k<l \leq n} \theta^{l} \Delta_{p-1}^{n-l} \frac{\rho_{l}}{\rho_{k}}\right] \\
& +\sum_{1 \leq k \leq n} R_{1}^{k}\left[\theta^{k} \Delta_{p-1}^{n-k} \theta^{k} \Delta_{q}^{n-k}+\theta^{k} \Delta_{p}^{n-k} \theta^{k} \Delta_{q-1}^{n-k}\right] .
\end{aligned}
$$


Taking $r \geq 2$, insert the latter in $K_{r}(n)=\sum_{-r+1 \leq p \leq r-1}(-1)^{p} \Delta_{r+p}^{n} \Delta_{r-p}^{n}+2(-1)^{r} \Delta_{2 r}^{n}$ and get:

$$
\begin{aligned}
& K_{r}(n)=\sum_{1 \leq k \leq n}\left(R_{1}^{k}\right)^{2} \sum_{-r+1 \leq p \leq r-1}(-1)^{p} \\
& {\left[\theta^{k} \Delta_{r+p-1}^{n-k} \sum_{k \leq l \leq n} \theta^{l} \Delta_{r-p-1}^{n-l} \frac{\rho_{l}}{\rho_{k}}+\theta^{k} \Delta_{r-p-1}^{n-k} \sum_{k<l \leq n} \theta^{l} \Delta_{r+p-1}^{n-l} \frac{\rho_{l}}{\rho_{k}}\right]} \\
& +2(-1)^{r} \Delta_{2 r}^{n}+2 \sum_{1 \leq k \leq n} R_{1}^{k}\left[\sum_{-r+1 \leq p \leq r-1}(-1)^{p} \theta^{k} \Delta_{r+p-1}^{n-k} \theta^{k} \Delta_{r-p}^{n-k}\right] .
\end{aligned}
$$

The last line is $2 \sum_{1 \leq k \leq n} R_{1}^{k}\left[\sum_{-r+1 \leq p \leq r}(-1)^{p} \theta^{k} \Delta_{r+p-1}^{n-k} \theta^{k} \Delta_{r-p}^{n-k}\right]$. Each bracketed sum is 0 , as can be seen for instance when doing the change of variable $p \longmapsto$ $-p+1$. Separating now the term with $k=l$ in the first sum above and recognizing $\theta^{k} K_{r-1}(n-k)$, we obtain:

$$
K_{r}(n)=\sum_{1 \leq k \leq n}\left(R_{1}^{k}\right)^{2}\left[\theta^{k} K_{r-1}(n-k)+2 \sum_{-r+1 \leq p \leq r-1}(-1)^{p} \theta^{k} \Delta_{r+p-1}^{n-k} \sum_{k<l \leq n} \theta^{l} \Delta_{r-p-1}^{n-l} \frac{\rho_{l}}{\rho_{k}}\right]
$$

Setting $m=n-k$ and $Z_{r}(m)=\sum_{-r \leq p \leq r}(-1)^{p} \Delta_{r+p}^{m} \sum_{1 \leq l \leq m} \theta^{l} \Delta_{r-p}^{m-l} \rho_{l}$, we therefore have:

$$
K_{r}(n)=\sum_{1 \leq k \leq n}\left(R_{1}^{k}\right)^{2}\left[\theta^{k} K_{r-1}(n-k)+2 \theta^{k} Z_{r-1}(n-k)\right] .
$$

We shall show that:

$$
Z_{r}(m)=\sum_{1 \leq k \leq m} \theta^{k} K_{r}(m-k) \rho_{k}, r \geq 1
$$

To complete the proof of (5.3), we simply apply this to $Z_{r-1}(n-k)$ in the previous equality. First of all, with $0 \leq p \leq r-1$ :

$$
\begin{aligned}
& \Delta_{r+p}^{m} \sum_{1 \leq l \leq m} \theta^{l} \Delta_{r-p}^{m-l} \rho_{l}=\sum_{\substack{1 \leq k_{1}<\cdots<k_{r+p} \leq m \\
1 \leq l_{1}<l_{2}<\cdots<l_{r-p+1} \leq m}} R_{1}^{k_{1}} \cdots R_{k_{r+p}+1}^{k_{r+p}} R_{l_{1}+1}^{l_{2}} \cdots R_{l_{r-p}+1}^{l_{r-p+1}} \rho_{l_{1}} \\
& =\sum_{\substack{1 \leq k_{1}<\cdots<k_{r+p} \leq m \\
k_{1} \leq l_{1}<\cdots<l_{r-p+1} \leq m}} R_{1}^{k_{1}} R_{k_{1}+1}^{k_{2}} \cdots R_{k_{r+p-1}+1}^{k_{r+p}} R_{l_{1}+1}^{l_{2}} \cdots R_{l_{r-p}+1}^{l_{r-p+1}} \rho_{l_{1}} \\
& +\sum_{\substack{1 \leq l_{1}<\cdots<l_{r-p+1} \leq m \\
l_{1}<k_{1}<\cdots<k_{r+p} \leq m}}\left(R_{1}^{l_{1}} \frac{\rho_{k_{1}}}{\rho_{l_{1}}}+R_{l_{1}+1}^{k_{1}}\right) R_{k_{1}+1}^{k_{2}} \cdots R_{k_{r+p-1}+1}^{k_{r+p}} R_{l_{1}+1}^{l_{2}} \cdots R_{l_{r-p}+1}^{l_{r-p+1}} \rho_{l_{1}} .
\end{aligned}
$$


Written in a more concise way:

$$
\begin{aligned}
& \Delta_{r+p}^{m} \sum_{1 \leq l \leq m} \theta^{l} \Delta_{r-p}^{m-l} \rho_{l} \\
& =\sum_{1 \leq k \leq m} R_{1}^{k}\left[\theta^{k} \Delta_{r+p-1}^{m-k} \sum_{k \leq l \leq m} \theta^{l} \Delta_{r-p}^{m-l} \rho_{l}+\theta^{k} \Delta_{r-p}^{m-k} \sum_{k<l \leq m} \theta^{l} \Delta_{r+p-1}^{m-l} \rho_{l}\right] \\
& +\sum_{1 \leq k \leq m} \theta^{k} \Delta_{r+p}^{m-k} \theta^{k} \Delta_{r-p}^{m-k} \rho_{k} .
\end{aligned}
$$

This allows to write:

$$
\begin{aligned}
& Z_{r}(m)=(-1)^{r}\left[\Delta_{2 r}^{m} \sum_{1 \leq l \leq m} \rho_{l}+\sum_{1 \leq l \leq m} \theta^{l} \Delta_{2 r}^{m-l} \rho_{l}\right] \\
& +\sum_{1 \leq k \leq m-r+1 \leq p \leq r-1} \sum_{(-1)^{p} \theta^{k} \Delta_{r+p}^{m-k} \theta^{k} \Delta_{r-p}^{m-k} \rho_{k}} \\
& +\sum_{1 \leq k \leq m} R_{1}^{k} \sum_{-r+1 \leq p \leq r-1}(-1)^{p}\left[\theta^{k} \Delta_{r+p-1}^{m-k} \sum_{k \leq l \leq m} \theta^{l} \Delta_{r-p}^{m-l} \rho_{l}+\theta^{k} \Delta_{r-p}^{m-k} \sum_{k<l \leq m} \theta^{l} \Delta_{r+p-1}^{m-l} \rho_{l}\right] .
\end{aligned}
$$

Recognizing some $\theta^{k} K_{r}(m-k)$, we get:

$$
\begin{aligned}
Z_{r}(m) & =(-1)^{r}\left[\Delta_{2 r}^{m} \sum_{1 \leq l \leq m} \rho_{l}-\sum_{1 \leq l \leq m} \theta^{l} \Delta_{2 r}^{m-l} \rho_{l}\right]+\sum_{1 \leq k \leq m} \theta^{k} K_{r}(m-k) \rho_{k} \\
& +\sum_{1 \leq k \leq m} R_{1}^{k} \sum_{-r+1 \leq p \leq r-1}(-1)^{p}\left[\theta^{k} \Delta_{r+p-1}^{m-k} \sum_{k \leq l \leq m} \theta^{l} \Delta_{r-p}^{m-l} \rho_{l}\right] \\
& +\sum_{1 \leq k \leq m} R_{1}^{k} \sum_{-r+2 \leq p \leq r}(-1)^{p+1}\left[\theta^{k} \Delta_{r+p-1}^{m-k} \sum_{k<l \leq m} \theta^{l} \Delta_{r-p}^{m-l} \rho_{l}\right] .
\end{aligned}
$$

Consequently:

$$
\begin{aligned}
& Z_{r}(m)=\sum_{1 \leq k \leq m} \theta^{k} K_{r}(m-k) \rho_{k}+(-1)^{r}\left[\Delta_{2 r}^{m} \sum_{1 \leq l \leq m} \rho_{l}-\sum_{1 \leq l \leq m} \theta^{l} \Delta_{2 r}^{m-l} \rho_{l}\right] \\
& +(-1)^{r+1} \sum_{1 \leq k \leq m} R_{1}^{k}\left(\theta^{k} \Delta_{2 r-1}^{m-k} \sum_{k \leq l \leq m} \rho_{l}+\sum_{k<l \leq m} \theta^{l} \Delta_{2 r-1}^{m-l} \rho_{l}\right) \\
& +\sum_{1 \leq k \leq m} R_{1}^{k} \sum_{-r+1 \leq p \leq r}(-1)^{p}\left[\theta^{k} \Delta_{r+p-1}^{m-k} \sum_{k \leq l \leq m} \theta^{l} \Delta_{r-p}^{m-l} \rho_{l}-\theta^{k} \Delta_{r+p-1}^{m-k} \sum_{k<l \leq m} \theta^{l} \Delta_{r-p}^{m-l} \rho_{l}\right] .
\end{aligned}
$$

The last line is $\sum_{1 \leq k \leq m} R_{1}^{k}\left[\sum_{-r+1 \leq p \leq r}(-1)^{p} \theta^{k} \Delta_{r+p-1}^{m-k} \theta^{k} \Delta_{r-p}^{m-k} \rho_{k}\right]$. For the same reason as before, the sums inside each brackets are 0 . Therefore it finally remains 
to show that the sum of the second and third terms is also 0, in other words that:

$$
\begin{aligned}
& \Delta_{2 r}^{m} \sum_{1 \leq l \leq m} \rho_{l}-\sum_{1 \leq l \leq m} \theta^{l} \Delta_{2 r}^{m-l} \rho_{l} \\
& -\sum_{1 \leq k \leq m} R_{1}^{k}\left(\theta^{k} \Delta_{2 r-1}^{m-k} \sum_{k \leq l \leq m} \rho_{l}+\sum_{k<l \leq m} \theta^{l} \Delta_{2 r-1}^{m-l} \rho_{l}\right)=0 .
\end{aligned}
$$

Equivalently:

$$
\sum_{1 \leq k \leq m} R_{1}^{k} \theta^{k} \Delta_{2 r-1}^{m-k} \sum_{1 \leq k<l} \rho_{l}-\sum_{1 \leq l \leq m} \theta^{l} \Delta_{2 r}^{m-l} \rho_{l}-\sum_{1 \leq k \leq m} R_{1}^{k} \sum_{k<l \leq m} \theta^{l} \Delta_{2 r-1}^{m-l} \rho_{l}=0 .
$$

In the last term, replace $R_{1}^{k}$ by $\left(R_{1}^{l}-R_{k+1}^{l}\right) \rho_{k} / \rho_{l}$. It remains to show that:

$$
\begin{aligned}
& -\sum_{1 \leq l \leq m} \theta^{l} \Delta_{2 r}^{m-l} \rho_{l}+\sum_{1 \leq k \leq m} R_{1}^{k} \theta^{k} \Delta_{2 r-1}^{m-k} \sum_{1 \leq l<k} \rho_{l} \\
& -\sum_{1 \leq k<l \leq m} R_{1}^{l} \theta^{l} \Delta_{2 r-1}^{m-l} \rho_{k}+\sum_{1 \leq k<l \leq m} R_{k+1}^{l} \theta^{l} \Delta_{2 r-1}^{m-l} \rho_{k}=0 .
\end{aligned}
$$

As this is true, this completes the proof of this first point.

2. Let us define $\tilde{\Delta}_{r}^{n}=\sum_{0 \leq k \leq n} \rho_{k} \theta^{k} \Delta_{r}^{n-k}$, so that $B_{n}(u t)=\sum_{0 \leq r \leq n}(-i t)^{r} \tilde{\Delta}_{r}^{n}$. As for $\left|B_{n}(u t)-A_{n}(u t)\right|^{2}$ in the first point, we have:

$$
\left|B_{n}(u t)\right|^{2}=\sum_{0 \leq r \leq n} t^{2 r} L_{r}(n), \text { where } L_{r}(n)=\sum_{-r \leq p \leq r}(-1)^{p} \tilde{\Delta}_{r+p}^{n} \tilde{\Delta}_{r-p}^{n} .
$$

In order to compute $L_{r}(n)$, notice first that:

$$
\tilde{\Delta}_{r+p}^{n} \tilde{\Delta}_{r-p}^{n}=\sum_{0 \leq k \leq n} \rho_{k}\left[\theta^{k} \Delta_{r+p}^{n-k} \sum_{k \leq l \leq n} \theta^{l} \Delta_{r-p}^{n-l} \rho_{l}+\theta^{k} \Delta_{r-p}^{n-k} \sum_{k<l \leq n} \theta^{l} \Delta_{r+p}^{n-l} \rho_{l}\right] .
$$

Replacing in $L_{r}(n)$, this allows to write, using the expressions of $K_{r}(n)$ and $Z_{r}(n)$ given in (5.4):

$$
\begin{aligned}
L_{r}(n) & =\sum_{0 \leq k \leq n} \rho_{k} \sum_{-r \leq p \leq r}(-1)^{p}\left[\theta^{k} \Delta_{r+p}^{n-k} \sum_{k \leq l \leq n} \rho_{l} \theta^{l} \Delta_{r-p}^{n-l}+\theta^{k} \Delta_{r-p}^{n-k} \sum_{k<l \leq n} \rho_{l} \theta^{l} \Delta_{r+p}^{n-l}\right] \\
& =\sum_{0 \leq k \leq n}\left(\rho_{k}\right)^{2} \theta^{k} K_{r}(n-k)+2 \sum_{0 \leq k \leq n}\left(\rho_{k}\right)^{2} \theta^{k} Z_{r}(n-k) \\
& =\sum_{0 \leq k \leq n} \rho_{k}\left[\rho_{k} \theta^{k} K_{r}(n-k)+2 \sum_{k<l \leq n} \rho_{l} \theta^{l} K_{r}(n-l)\right] \\
& =\sum_{0 \leq k \leq n} \rho_{k} \theta^{k} K_{r}(n-k) \sum_{0 \leq l \leq k} 2^{(l, k)} \rho_{l} .
\end{aligned}
$$

This completes the proof of this point.

3. Directly, we obtain:

$$
\left(B_{n}-A_{n}\right)(u t) \bar{B}_{n}(u t)=\sum_{0 \leq r \leq n}(-i t)^{r} \Delta_{r}^{n} \sum_{0 \leq r^{\prime} \leq n}(i t)^{r^{\prime}} \tilde{\Delta}_{r^{\prime}}^{n}
$$


When developing and taking the real part, only terms with $r+r^{\prime}$ even intervene. This gives:

$$
\begin{aligned}
\operatorname{Re}\left(\left(B_{n}-A_{n}\right) \bar{B}_{n}\right)(u t) & =\sum_{0 \leq r \leq n} t^{2 r}\left[\sum_{-r \leq p \leq r}(-i)^{r+p} i^{r-p} \Delta_{r+p}^{n} \tilde{\Delta}_{r-p}^{n}\right] \\
& =\sum_{0 \leq r \leq n} t^{2 r} M_{r}(n),
\end{aligned}
$$

with this time:

$$
M_{r}(n)=\sum_{-r \leq p \leq r}(-1)^{p} \Delta_{r+p}^{n} \tilde{\Delta}_{r-p}^{n} .
$$

Since $\tilde{\Delta}_{r}^{n}=\Delta_{r}^{n}+\sum_{1 \leq k \leq n} \rho_{k} \theta^{k} \Delta_{r}^{n-k}$, using $K_{r}(n)$ and the value of $Z_{r}(n)$ in (5.4), we have:

$$
M_{r}(n)=K_{r}(n)+Z_{r}(n)=\sum_{0 \leq k \leq n} \rho_{k} \theta^{k} K_{r}(n-k) .
$$

This ends the proof of this point.

4. In the same way as for 3., when taking the imaginary part in (5.6), only terms with $r+r^{\prime}$ odd come into play. Consequently:

$$
\begin{aligned}
\operatorname{Im}\left(A_{n} \bar{B}_{n}\right)(u t) & =-\frac{1}{i} \sum_{0 \leq r \leq n-1} t^{2 r+1}\left[\sum_{-r-1 \leq p \leq r}(-i)^{r+p+1} i^{r-p} \Delta_{r+p+1}^{n} \tilde{\Delta}_{r-p}^{n}\right] \\
& =\sum_{0 \leq r \leq n-1} t^{2 r+1} N_{r}(n),
\end{aligned}
$$

with this time:

$$
N_{r}(n)=\sum_{-r-1 \leq p \leq r}(-1)^{p} \Delta_{r+p+1}^{n} \tilde{\Delta}_{r-p}^{n} .
$$

Using again that $\tilde{\Delta}_{r}^{n}=\sum_{0 \leq k \leq n} \rho_{k} \theta^{k} \Delta_{r}^{n-k}$, we get:

$$
N_{r}(n)=\sum_{0 \leq k \leq n} \sum_{-r-1 \leq p \leq r}(-1)^{p} \Delta_{r+p+1}^{n} \theta^{k} \Delta_{r-p}^{n-k} \rho_{k} .
$$

Notice that the term corresponding to $k=0$ equals 0 , for symmetry reasons as before. It remains:

$$
\begin{aligned}
N_{r}(n) & =(-1)^{r+1} \sum_{1 \leq k \leq n} \theta^{k} \Delta_{2 r+1}^{n-k} \rho_{k}+\sum_{1 \leq l \leq n} R_{1}^{l} \sum_{-r \leq p \leq r}(-1)^{p} \theta^{l} \Delta_{r+p}^{n-l} \sum_{l<k \leq n} \theta^{k} \Delta_{r-p}^{n-k} \rho_{k} \\
& +\sum_{1 \leq k \leq n} \sum_{-r \leq p \leq r}(-1)^{p} \theta^{k} \Delta_{r-p}^{n-k} \rho_{k} \sum_{k \leq l \leq n} R_{1}^{l} \theta^{l} \Delta_{r+p}^{n-l} \\
& =\sum_{1 \leq k \leq n} R_{1}^{k} \rho_{k} \theta^{k} K_{r}(n-k)+\sum_{1 \leq l \leq n} R_{1}^{l} \rho_{l} \theta^{l} Z_{r}(n-l)+O_{r}(n)
\end{aligned}
$$

where we introduce:

$O_{r}(n)=(-1)^{r+1} \sum_{1 \leq k \leq n} \theta^{k} \Delta_{2 r+1}^{n-k} \rho_{k}+\sum_{1 \leq k \leq n} \sum_{-r \leq p \leq r}(-1)^{p} \theta^{k} \Delta_{r-p}^{n-k} \rho_{k} \sum_{k<l \leq n} R_{1}^{l} \theta^{l} \Delta_{r+p}^{n-l}$. 
To compute $O_{r}(n)$, in the last sum decompose $R_{1}^{l}=R_{1}^{k}\left(\rho_{l} / \rho_{k}\right)+R_{k+1}^{l}$. As a result:

$$
\begin{aligned}
O_{r}(n) & =\sum_{1 \leq k \leq n} R_{1}^{k} \sum_{-r \leq p \leq r}(-1)^{p} \theta^{k} \Delta_{r-p}^{n-k} \sum_{k<l \leq n} \theta^{l} \Delta_{r+p}^{n-l} \rho_{l} \\
& +(-1)^{r+1} \sum_{1 \leq k \leq n} \theta^{k} \Delta_{2 r+1}^{n-k} \rho_{k}+\sum_{1 \leq k \leq n} \sum_{-r \leq p \leq r}(-1)^{p} \theta^{k} \Delta_{r-p}^{n-k} \theta^{k} \Delta_{r+p+1}^{n-k} \rho_{k} \\
& =\sum_{1 \leq k \leq n} R_{1}^{k} \theta^{k} Z_{r}(n-k) \rho_{k}+\sum_{1 \leq k \leq n-r \leq p \leq r-1} \sum_{-1)^{p} \theta^{k} \Delta_{r-p}^{n-k} \theta^{k} \Delta_{r+p+1}^{n-k} \rho_{k} .}
\end{aligned}
$$

One more time, the last term is 0 . Together with (5.7) and (5.4) we obtain:

$$
N_{r}(n)=\sum_{1 \leq k \leq n} \rho_{k} R_{1}^{k}\left(\theta^{k} K_{r}(n-k)+2 \theta^{k} Z_{r}(n-k)\right)=\sum_{1 \leq k \leq l \leq n} R_{1}^{k} \theta^{l} K_{r}(n-l) \rho_{l} 2^{(k, l)} .
$$

This gives the announced formula and concludes the proof of the proposition.

\section{Proof of the theorem}

6.1. Definitions; dominated variation. We next introduce some definitions concerning directional fluxes and their variations.

\section{Definition 6.1.}

1) For $m \geq 0, n \geq 0$, define $F(-m, n)=\left(n w_{+} \circ v_{+}^{-1}(n)+m w_{-} \circ v_{-}^{-1}(m)\right)^{1 / 2}$. Staying coherent with the previous definition of $F_{+}$, set:

$$
F(n)=F(-n, n), F_{+}(n)=F(0, n), F_{-}(n)=F(-n, 0) .
$$

2) For $u \in S_{+}^{d-1}, m \geq 0, n \geq 0$, let:

$$
G_{u}(-m, n)=\left(F^{2}(-m, n)+\sum_{-v_{-}^{-1}(m) \leq k \leq l \leq v_{+}^{-1}(n)} T_{k}^{l}(u)\right)^{1 / 2} .
$$

Introduce for $n \geq 0$ :

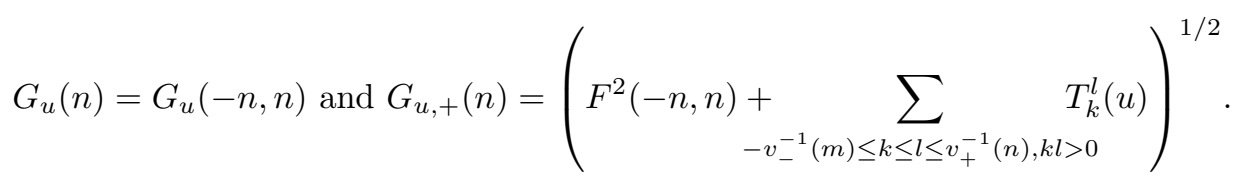

A central point for what follows, is that the inverse functions $n \rightarrow G_{u,+}^{-1}(n)$ and $n \rightarrow G_{u}^{-1}(n)$ check a dominated variation property at infinity. This notion has been introduced by Feller in 1969. A non-decreasing map $f: \mathbb{R}_{+} \rightarrow \mathbb{R}_{+}$verifies dominated variation if:

$$
\exists K>0, \forall x>0, f(2 x) \leq K f(x) .
$$

By iteration, for any $C \geq 1$, then $\forall x>0, f(C x) \leq K^{\prime} f(x)$, for some constant $K^{\prime}$. Notice that dominated variation holds for $F_{+}^{-1}$ and $F_{-}^{-1}$, as shown in the first point of lemma 4.8.

\section{Lemma 6.2.}

1. For any $x \geq 1$ and $K \geq 1$ :

$$
F^{-1}(K x) \leq 2 K^{2} F^{-1}(x)
$$


2. There exists a constant $C(\delta)>0$, so that for any $u \in S_{+}^{d-1}$, any $x \geq 1$ and $K \geq 1$ :

$$
G_{u,+}^{-1}(K x) \leq \frac{2 K^{2}}{\delta} G_{u,+}^{-1}(x) \text { and } G_{u}^{-1}(K x) \leq \frac{K^{2}}{C(\delta)} G_{u}^{-1}(x) .
$$

Proof: 1 . We have $F^{2}(n)=n\left(w_{+}(n) \circ v_{+}^{-1}(n)+w_{-}(n) \circ v_{-}^{-1}(n)\right)$. For $x \geq 1$, let $n=F^{-1}(x)$, ie $F(n) \leq x<F(n+1)$. This implies that:

$$
F\left(K^{2}(n+1)\right) \geq K F(n+1)>K x .
$$

Hence $F^{-1}(K x) \leq K^{2}(n+1) \leq 2 K^{2} n=2 K^{2} F^{-1}(x)$.

2. Let $\zeta_{k}^{l}(u)=\sum_{s=k}^{l} \eta_{s}^{u} / \rho_{s}$, with $\zeta_{k}^{l}(u)=0$ if $k>l$. Set:

$$
\kappa_{u,+}(n)=\sum_{1 \leq k \leq l \leq n} T_{k}^{l}(u)=\sum_{0 \leq k<l \leq n} \rho_{k} \rho_{l}\left(\zeta_{k+1}^{l}(u)\right)^{2} .
$$

We first claim that:

$$
\frac{\kappa_{u,+}(n)}{v_{+}(n)}=\frac{\kappa_{u,+}(n-1)}{v_{+}(n-1)}+\frac{\rho_{n}}{v_{+}(n) v_{+}(n-1)}\left(\sum_{0 \leq k<n} \rho_{k} \zeta_{k+1}^{n}(u)\right)^{2} .
$$

In particular, this shows that $n \longmapsto \kappa_{u,+}(n) / v_{+}(n)$ is non-decreasing. Indeed:

$$
\begin{aligned}
\kappa_{u,+}(n)= & \sum_{0 \leq k<l \leq n} \rho_{k} \rho_{l}\left(\zeta_{k+1}^{n}(u)\right)^{2}+\sum_{0 \leq k<l \leq n} \rho_{k} \rho_{l}\left(\zeta_{l+1}^{n}(u)\right)^{2} \\
& -2 \sum_{0 \leq k<l \leq n} \rho_{k} \rho_{l} \zeta_{k+1}^{n}(u) \zeta_{l+1}^{n}(u) .
\end{aligned}
$$

This is rewritten as:

$$
\begin{aligned}
\kappa_{u,+}(n) & =\sum_{0 \leq k<n} \rho_{k}\left(\zeta_{k+1}^{n}(u)\right)^{2} \sum_{k<l \leq n} \rho_{l}+\sum_{1 \leq l \leq n} \rho_{l}\left(\zeta_{l+1}^{n}(u)\right)^{2} \sum_{0 \leq k<l} \rho_{k} \\
& -\left(\sum_{0 \leq k \leq n} \rho_{k} \zeta_{k+1}^{n}(u)\right)^{2}+\sum_{0 \leq k \leq n}\left(\rho_{k}\right)^{2}\left(\zeta_{k+1}^{n}(u)\right)^{2} .
\end{aligned}
$$

In other words, using that $\zeta_{n+1}^{n}(u)=0$ :

$$
\kappa_{u,+}(n)=v_{+}(n) \sum_{0 \leq k<n} \rho_{k}\left(\zeta_{k+1}^{n}(u)\right)^{2}-\left(\sum_{0 \leq k<n} \rho_{k} \zeta_{k+1}^{n}(u)\right)^{2} .
$$

Next, directly from the definition of $\kappa_{u,+}(n)$, and then using the previous equality:

$$
\begin{aligned}
\kappa_{u,+}(n)-\kappa_{u,+}(n-1) & =\rho_{n} \sum_{0 \leq k<n} \rho_{k}\left(\zeta_{k+1}^{n}(u)\right)^{2} \\
& =\rho_{n} \frac{\kappa_{u,+}(n)+\left(\sum_{0 \leq k<n} \rho_{k} \zeta_{k+1}^{n}(u)\right)^{2}}{v_{+}(n)} .
\end{aligned}
$$

Observe that this is equivalent to the desired claim. 
We next use that for all $n \geq 0, \delta \leq \rho_{n+1} / \rho_{n} \leq 1 / \delta$, giving $v_{+}(n+1) \leq$ $(2 / \delta) v_{+}(n)$. As a result $v_{+} \circ v_{+}^{-1}(n) \leq n \leq(2 / \delta) v_{+} \circ v_{+}^{-1}(n)$. Hence for $x \geq 1$ and $K \geq 1$ :

$$
\kappa_{u,+} \circ v_{+}^{-1}(K x) \geq \kappa_{u,+} \circ v_{+}^{-1}(x) \frac{v_{+} \circ v_{+}^{-1}(K x)}{v_{+} \circ v_{+}^{-1}(x)} \geq \frac{\delta K}{2} \kappa_{u,+} \circ v_{+}^{-1}(x) .
$$

A similar property is verified for some symmetrically defined function $\kappa_{u,-} \circ v_{-}^{-1}$. Notice that:

$$
G_{u,+}^{2}(n)=F^{2}(n)+\kappa_{u,+} \circ v_{+}^{-1}(n)+\kappa_{u,-} \circ v_{-}^{-1}(n) .
$$

Remark that $G_{u,+}(n) \rightarrow+\infty$, as $n \rightarrow+\infty$. We showed in point one that $F^{2}(K x) \geq$ $K F^{2}(x)$. Putting everything together, we obtain that for $x \geq 1$ and $K \geq 1$ :

$$
G_{u,+}(K x) \geq \sqrt{(\delta K / 2)} G_{u,+}(x) .
$$

We conclude as in point one. Let next $x \geq 1, n=G_{u,+}^{-1}(x)$ and $K \geq 1$. From the relation $G_{u,+}(n) \leq x<G_{u,+}(n+1)$, we deduce:

$$
G_{u,+}\left(\left(2 K^{2} / \delta\right)(n+1)\right) \geq K G_{u,+}(n+1)>K x .
$$

Consequently $G_{u,+}^{-1}(K x) \leq\left(\left(2 K^{2}\right) / \delta\right) G_{u,+}^{-1}(x)$.

It finally remains to show the same result for $G_{u}$. This way, let $\kappa_{u}(-m, n)=$ $\sum_{-m \leq k \leq l \leq n} T_{k}^{l}(u)$, for $m \geq 1, n \geq 1$. Then, the computation on $\kappa_{u,+}$ shows that:

$$
n \longmapsto \frac{\kappa_{u}(-m, n)}{\left(v_{-}(m) / a_{0}\right)+v_{+}(n)} \text { and } m \longmapsto \frac{\kappa_{u}(-m, n)}{\left(v_{-}(m) / a_{0}\right)+v_{+}(n)}
$$

are non-decreasing. This furnishes that for some constant $C(\delta)>0$ :

$$
\kappa_{u}\left(-v_{-}^{-1}(K x), v_{+}^{-1}(K x)\right) \geq \frac{C(\delta) K}{2} \kappa_{u}\left(-v_{-}^{-1}(x), v_{+}^{-1}(x)\right) .
$$

As $G_{u}^{2}(n)=F^{2}(n)+\kappa_{u}\left(-v_{-}^{-1}(n), v_{+}^{-1}(n)\right)$, we conclude once again as before. This ends the proof of the lemma.

Remark 6.3. As a consequence of the previous lemma, we can work with functions verifying dominated variation up to multiplicative positive constants, outside the function or in the argument. This will appear clearly. This will for example allow to use frequently that, when $f=g+h$, all positive, non-decreasing and tending to $+\infty$ that for $x>0$ :

$$
f(x) \asymp \max \{g(x), h(x)\}, f^{-1}(x) \asymp \min \left\{g^{-1}(x), h^{-1}(x)\right\}, \text { etc. }
$$

6.2. Order of $\operatorname{Re}\left(1-\chi_{D}(u t)\right)$. With $u \in S_{+}^{d-1}$ and small $t>0$, recall from relation (4.6) the decomposition:

$$
\chi_{D}(u t)=\varphi_{0}(u t)\left(p_{0}^{\prime} \chi_{D}^{+}(u t)+q_{0}^{\prime} \chi_{D}^{-}(u t)\right) .
$$

Also from lemma 4.8 we have that:

$$
\chi_{D}^{+}(u t)=f^{+}(u t)+O\left(R^{+}(t)\right) \text { and } \chi_{D}^{-}(u t)=f^{-}(u t)+O\left(R^{-}(t)\right),
$$

where the $O\left(\right.$ ) are uniform in $u \in S_{+}^{d-1}$. The terms $R^{+}(t)$ and $R^{-}(t)$ have respective orders $1 / F_{+}^{-1}(1 / t)$ and $1 / F_{-}^{-1}(1 / t)$, by lemma 4.8 .

Definition 6.4. Let $R(t)=R^{+}(t)+R^{-}(t)$.

The main preliminary result for estimating $\operatorname{Re}\left(1-\chi_{D}(u t)\right)$ is as follows. 


\section{Lemma 6.5.}

1. We have $\chi_{D}(u t)=\varphi_{0}(u t)\left(p_{0}^{\prime} f^{+}(u t)+q_{0}^{\prime} f^{-}(u t)\right)+O(R(t))$.

2. We have $t^{2}=O\left(R^{+}(t)\right)$ and $t^{2}=O\left(R^{-}(t)\right)$.

3. We have $\operatorname{Im}\left(1-f^{+}(u t)\right)=O\left(R^{+}(t)\right)$ and $t \operatorname{Im}\left(1-f^{-}(u t)\right)=O\left(R^{-}(t)\right)$.

4. We have $\chi_{D}(u t)=\left(1+i t m_{0} . u r_{0} /\left(1-r_{0}\right)\right)\left(p_{0}^{\prime} f^{+}(u t)+q_{0}^{\prime} f^{-}(u t)\right)+O(R(t))$ and

$$
\operatorname{Re}\left(1-\chi_{D}\right)(u t)=p_{0}^{\prime} \operatorname{Re}\left(1-f^{+}(u t)\right)+q_{0}^{\prime} \operatorname{Re}\left(1-f^{-}(u t)\right)+O(R(t)) .
$$

5. There is a constant $c>0$ so that for small $t>0$, uniformly in $u \in S_{+}^{d-1}$ :

$$
\operatorname{Re}\left(1-\chi_{D}(u t)\right) \geq c R(t) .
$$

Proof:

1. This follows from $\chi_{D}(u t)=\varphi_{0}(u t)\left(p_{0}^{\prime} \chi_{D}^{+}(u t)+q_{0}^{\prime} \chi_{D}^{-}(u t)\right)$ and $\chi_{D}^{ \pm}(u t)=f^{ \pm}(u t)+$ $O\left(R^{ \pm}(t)\right)$.

2. As $F_{+}^{2}(n)=n w_{+} \circ v_{+}^{-1}(n)$, for some constant $c>0$, we get $F_{+}\left(n^{2}\right) \geq c n$. As a result, $F_{+}^{-1}(1 / t) \leq c^{\prime} / t^{2}, t>0$, for another constant $c^{\prime}>0$. Thus $t^{2}=$ $O\left(1 / F_{+}^{-1}(1 / t)\right)=O\left(R_{+}(t)\right)$, using finally lemma 4.8. This gives the first property. The other one is proved in the same way.

3. We make use of proposition 5.2 and lemma 3.1. Take any integer $n \geq 1$. Since $f^{+}(u t)=A_{n}(u t) / B_{n}(u t)+O\left(1 / v_{+}(n)\right)$ (where $O()$ is independent on $u$ and $t$ ), we have:

$$
\begin{aligned}
\operatorname{Im}\left(f^{+}(u t)\right) & =\frac{\left.\operatorname{Im}\left(A_{n}(u t)\right) \bar{B}_{n}(u t)\right)}{\left|B_{n}(u t)\right|^{2}}+O\left(1 / v_{+}(n)\right) \\
& =\frac{\sum_{0 \leq r \leq n-1} t^{2 r+1} N_{r}^{u}(n)}{\sum_{0 \leq r \leq n} t^{2 r} L_{r}^{u}(n)}+O\left(1 / v_{+}(n)\right) .
\end{aligned}
$$

Now, observe that:

$$
\begin{gathered}
L_{r}^{u}(n)=\sum_{0 \leq l \leq k \leq n} \rho_{k} \theta^{k} K_{r}^{u}(n-k) 2^{(l, k)} \rho_{l} \geq \sum_{1 \leq k \leq n} \rho_{k} v_{+}(k) \theta^{k} K_{r}^{u}(n-k) \text { and } \\
N_{r}^{u}(n)=\sum_{1 \leq k \leq l \leq n} R_{1}^{k} \theta^{l} K_{r}^{u}(n-l) \rho_{l} 2^{(k, l)}=\sum_{1 \leq k \leq n}\left[\sum_{1 \leq s \leq l \leq k} \frac{\eta_{s}^{u}}{\rho_{s}} \rho_{l} 2^{(l, k)}\right] \rho_{k} \theta^{k} K_{r}^{u}(n-k) .
\end{gathered}
$$

As the $\eta_{n}^{u}$ are uniformly bounded by some $C / 2$, as $n$ and $u \in S_{+}^{d-1}$ vary, we get:

$$
\left|N_{r}^{u}(n)\right| \leq C w_{+}(n) \sum_{1 \leq k \leq n} v_{+}(k) \rho_{k} \theta^{k} K_{r}^{u}(n-k) \leq C w_{+}(n) L_{r}^{u}(n) .
$$

We finally obtain:

$$
\left|\operatorname{Im}\left(f^{+}(u t)\right)\right| \leq t w_{+}(n)+O\left(1 / v_{+}(n)\right) .
$$

Let $n^{\prime}=F_{+}^{-1}(1 / t)$ and $n=v_{+}^{-1}\left(n^{\prime}\right)$. By definition of $F_{+}$, we have $n^{\prime} w_{+}(n) \leq 1 / t^{2}$. We obtain $\left|\operatorname{Im}\left(f^{+}(u t)\right)\right| \leq 1 /\left(t n^{\prime}\right)+O\left(1 / n^{\prime}\right)$. This is the desired result. The case of $t\left|\operatorname{Im}\left(f^{-}(u t)\right)\right|$ is similar.

4. Write $\varphi_{0}(u t)=1+i t m_{0} \cdot u r_{0} /\left(1-r_{0}\right)+O\left(t^{2}\right)$, with $O()$ uniform in $u \in S_{+}^{d-1}$. Using the second point of the lemma, we get:

$$
\chi_{D}(u t)=\left(1+\frac{i t m_{0} \cdot u r_{0}}{1-r_{0}}\right)\left(p_{0}^{\prime} f^{+}(u t)+q_{0}^{\prime} f^{-}(u t)\right)+O(R(t)),
$$


with again an error term uniform in $u \in S_{+}^{d-1}$. Therefore:

$$
\begin{aligned}
1-\chi_{D}(u t)= & p_{0}^{\prime}\left(1-f^{+}(u t)\right)+q_{0}^{\prime}\left(1-f^{-}(u t)\right) \\
& -\frac{i t m_{0} \cdot u r_{0}}{1-r_{0}}\left(p_{0}^{\prime} f^{+}(u t)+q_{0}^{\prime} f^{-}(u t)\right)+O(R(t)) .
\end{aligned}
$$

Taking the real part:

$$
\begin{aligned}
\operatorname{Re}\left(1-\chi_{D}(u t)\right) & =p_{0}^{\prime} \operatorname{Re}\left(1-f^{+}(u t)\right)+q_{0}^{\prime} \operatorname{Re}\left(1-f^{-}(u t)\right) \\
& +\frac{t m_{0} \cdot u r_{0}}{1-r_{0}}\left(p_{0}^{\prime} \operatorname{Im}\left(f^{+}(u t)\right)+q_{0}^{\prime} \operatorname{Im}\left(f^{-}(u t)\right)\right)+O(R(t)) .
\end{aligned}
$$

The third point of the lemma then gives (6.1).

5. By lemma 4.8, for a constant $c_{1}>0$ independent on $u \in S_{+}^{d-1}$, we have for small $t>0,1-\left|\chi_{D}^{+}(u t)\right| \geq c_{1} R^{+}(u t)$. Idem, for some $c_{2}>0$, we get $1-\left|\chi_{D}^{-}(u t)\right| \geq$ $c_{2} R^{-}(u t)$. As $\chi_{D}(u t)=\varphi_{0}(u t)\left(p_{0}^{\prime} \chi_{D}^{+}(u t)+q_{0}^{\prime} \chi_{D}^{-}(u t)\right)$ and $\left|\varphi_{0}(u t)\right| \leq 1$ :

$$
\begin{aligned}
\operatorname{Re}\left(1-\chi_{D}(u t)\right) \geq 1-\left|\chi_{D}(u t)\right| & \geq 1-\left|p_{0}^{\prime} \chi_{D}^{+}(u t)+q_{0}^{\prime} \chi_{D}^{-}(u t)\right| \\
& \geq p_{0}^{\prime}\left(1-\left|\chi_{D}^{+}(u t)\right|\right)+q_{0}^{\prime}\left(1-\left|\chi_{D}^{-}(u t)\right|\right) \\
& \geq c_{1} p_{0}^{\prime} R^{+}(t)+c_{2} q_{0}^{\prime} R^{-}(t) \geq c R(t),
\end{aligned}
$$

for some constant $c>0$. This completes the proof of the lemma.

Remark 6.6. It may be noticed that in the flat case treated in Brémont (2016) one always had $t=O\left(R^{+}(t)\right)$. This is not true anymore here. For example if $\sum_{k \geq 1}\left(1 / \rho_{k}\right)<\infty$, then $F_{+}(n)$ has order $\sqrt{n}$, so that $R^{+}(t)$ has order $t^{2}$, as $t \rightarrow 0$.

The main result of this section is the following one.

Proposition 6.7. There is a constant $C \geq 1$ so that for $t>0$ small enough, uniformly in $u \in S_{+}^{d-1}$ :

$$
\frac{1}{C} \leq G_{u,+}^{-1}(1 / t) \operatorname{Re}\left(1-\chi_{D}(u t)\right) \leq C .
$$

Proof: We still fix $u \in S_{+}^{d-1}$ and $t>0$. Recall that $f^{+}(u t)=\lim _{n \rightarrow+\infty} A_{n}(u t) / B_{n}(u t)$, where $\left(B_{n}(u t)\right)$ and $\left(A_{n}(u t)\right)$ check relation (5.1) with $A_{n}(u t)=a_{1} \theta B_{n-1}(u t)$ and satisfy proposition 5.2 .

Fixing some $n \geq 1$, we use proposition 5.2 and lemma 3.1:

$$
\begin{aligned}
& \operatorname{Re}\left(1-f^{+}(u t)\right) \\
= & \operatorname{Re}\left(1-A_{n}(u t) / B_{n}(u t)\right)-\operatorname{Re}\left(\sum_{k>n} \frac{\rho_{k}}{B_{k}(u t) B_{k-1}(u t)}\right) \\
\leq & \frac{\operatorname{Re}\left(\left(B_{n}(u t)-A_{n}(u t)\right) \bar{B}_{n}(u t)\right)}{\left|B_{n}(u t)\right|^{2}}+\frac{1}{v_{+}(n)} \\
\leq & \frac{v_{+}(n)+\sum_{1 \leq r \leq n} t^{2 r} M_{r}^{u}(n)}{v_{+}(n)^{2}+\sum_{r=1}^{n} t^{2 r} L_{r}^{u}(n)}+\frac{1}{v_{+}(n)} \leq \frac{1}{v_{+}(n)}\left(2+\sum_{1 \leq r \leq n} t^{2 r} \frac{M_{r}^{u}(n)}{v_{+}(n)}\right),
\end{aligned}
$$

By the formula for $M_{r}^{u}(n)$ and $K_{r}^{u}(n)$ given in proposition 5.2, we have the inequalities $M_{r}^{u}(n) \leq\left(\sum_{1 \leq k \leq l \leq n} T_{k}^{l}(u)\right)^{r} 2^{r} v_{+}(n)$, for $r \geq 1$. Hence:

$$
M_{r}^{u}\left(v_{+}^{-1}(n)\right) \leq n 2^{r} G_{u,+}^{2 r}(n) .
$$


As a result, for some constant $C>0$ independent on $u$ and any $n \geq 1$ :

$$
\operatorname{Re}\left(1-f^{+}(u t)\right) \leq \frac{C}{n}\left[1+\sum_{1 \leq r \leq v_{+}^{-1}(n)}\left(2 t^{2}\right)^{r} G_{u,+}^{2 r}(n)\right] .
$$

Choose $n=n_{u}(t)=G_{u,+}^{-1}(1 /(2 t))$. In particular $G_{u,+}^{2}(n) \leq 1 /\left(4 t^{2}\right)$. We arrive at:

$$
\operatorname{Re}\left(1-f^{+}(u t)\right) \leq \frac{C}{n}\left(1+\sum_{r \geq 1} 2^{-r}\right) \leq \frac{2 C}{n}=\frac{2 C}{G_{u,+}^{-1}(1 /(2 t))} \leq \frac{C^{\prime}}{G_{u,+}^{-1}(1 / t)},
$$

for some constant $C^{\prime}$ independent on $u$, using lemma 6.2. Idem, $\operatorname{Re}\left(1-f^{-}(u t)\right) \leq$ $C^{\prime} / G_{u,+}^{-1}(1 / t)$. Now, by (6.1) and using that:

$$
R^{ \pm}(t)=O\left(1 / F_{ \pm}^{-1}(1 / t)\right)=O\left(1 / G_{u,+}^{-1}(1 / t)\right),
$$

we obtain the right-hand side inequality of the proposition.

Consider next the other direction. Starting in the same way, for any $n \geq 1$, via proposition 5.2 and lemma 3.1 (third point):

$$
\begin{aligned}
\operatorname{Re}\left(1-f^{+}(u t)\right) & =\operatorname{Re}\left(1-A_{n}(u t) / B_{n}(u t)\right)-\operatorname{Re}\left(\sum_{k>n} \frac{\rho_{k}}{B_{k}(u t) B_{k-1}(u t)}\right) \\
& \geq \frac{\operatorname{Re}\left(\left(B_{n}(u t)-A_{n}(u t)\right) \bar{B}_{n}(u t)\right)}{\left|B_{n}(u t)\right|^{2}}-\frac{v_{+}(n)}{\left|B_{n}(u t)\right|^{2}} \\
& =\frac{v_{+}(n)+\sum_{1 \leq r \leq n} t^{2 r} M_{r}^{u}(n)}{\left|B_{n}(u t)\right|^{2}}-\frac{v_{+}(n)}{\left|B_{n}(u t)\right|^{2}} \\
& =\frac{\sum_{1 \leq r \leq n} t^{2 r} M_{r}^{u}(n)}{v_{+}(n)^{2}+\sum_{1 \leq r \leq n} t^{2 r} L_{r}^{u}(n)} .
\end{aligned}
$$

By proposition 5.2:

$$
M_{r}^{u}(n)=\sum_{0 \leq k \leq n} \rho_{k} \theta^{k} K_{r}^{u}(n-k) \text { and } L_{r}^{u}(n)=\sum_{0 \leq l \leq k \leq n} 2^{(l, k)} \rho_{l} \rho_{k} \theta^{k} K_{r}^{u}(n-k) .
$$

Therefore we have $L_{r}^{u}(n) \leq 2 v_{+}(n) M_{r}^{u}(n)$. Hence, using in the last step below that $x \longmapsto x /(1+2 x)$ is increasing $(x>0)$ :

$$
\begin{aligned}
\operatorname{Re}\left(1-f^{+}(u t)\right) & \geq \frac{1}{v_{+}(n)} \frac{\sum_{1 \leq r \leq n} t^{2 r} M_{r}^{u}(n) / v_{+}(n)}{1+2 \sum_{1 \leq r \leq n} M_{r}^{u}(n) / v_{+}(n)} \\
& \geq \frac{1}{v_{+}(n)} \frac{t^{2} M_{1}^{u}(n) / v_{+}(n)}{1+2 t^{2} M_{1}^{u}(n) / v_{+}(n)},
\end{aligned}
$$

As a result, for some constant $c>0$ independent on $u$ and all $n \geq 1$ :

$$
\operatorname{Re}\left(1-f^{+}(u t)\right) \geq \frac{c}{n} \frac{c t^{2} M_{1}^{u}\left(v_{+}^{-1}(n)\right) / n}{1+2 c t^{2} M_{1}^{u}\left(v_{+}^{-1}(n)\right) / n} .
$$

Let $\kappa_{u,+}(m)=\sum_{1 \leq k \leq l \leq m} T_{k}^{l}(u)$. Assume first that $\lim _{m \rightarrow+\infty} \kappa_{u,+}(m)=+\infty$. Note, using proposition 5.2 , that:

$$
M_{1}^{u}(n) \geq \sum_{1 \leq m \leq n} \rho_{m} \kappa_{u,+}(m), \text { so } M_{1}^{u}\left(v_{+}^{-1}(n)\right) \geq \sum_{1 \leq m \leq v_{+}^{-1}(n)} \rho_{m} \kappa_{u,+}(m) .
$$


Let $c_{0} \geq 2$ be such that for all $n, v_{+}(n+1) \leq c_{0} v_{+}(n)$. Notice that $\sup _{v \in S_{+}^{d-1}}\left\{\kappa_{v,+} \circ\right.$ $\left.v_{+}^{-1}(1)\right\}$ is evidently finite, so $\left(\kappa_{u,+} \circ v_{+}^{-1}\right)^{-1}\left(1 / t^{2}\right) \geq 1$ for $t$ small enough, uniformly in $u \in S_{+}^{d-1}$.

For small $t$, set $m_{u}(t)=\left(\kappa_{u,+} \circ v_{+}^{-1}\right)^{-1}\left(1 / t^{2}\right) \geq 1$ and next choose $n_{u}(t)=$ $c_{0}^{2}\left(m_{u}(t)+1\right)$. Let $s=v_{+}^{-1}\left(m_{u}(t)+1\right)$ and $s^{\prime}=v_{+}^{-1}\left(n_{u}(t)\right)$. This gives:

$$
\begin{aligned}
& v_{+}(s) \leq m_{u}(t)+1<v_{+}(s+1) \leq c_{0} v_{+}(s) \text { and } \\
& v_{+}\left(s^{\prime}\right) \leq c_{0}^{2}\left(m_{u}(t)+1\right)<v_{+}\left(s^{\prime}+1\right) \leq c_{0} v_{+}\left(s^{\prime}\right) .
\end{aligned}
$$

As a result, $c_{0}^{2}\left(m_{u}(t)+1\right) \geq v_{+}\left(s^{\prime}\right)-v_{+}(s) \geq c_{0}\left(m_{u}(t)+1\right)-\left(m_{u}(t)+1\right) \geq$ $\left(c_{0}-1\right)\left(m_{u}(t)+1\right)$. Also $m_{u}(t)+1 \geq v_{+}(s) \geq\left(m_{u}(t)+1\right) / c_{0}$. This furnishes the inequalities:

$$
\begin{aligned}
\frac{M_{1}^{u}\left(v_{+}^{-1}\left(n_{u}(t)\right)\right)}{n_{u}(t)} & \geq \frac{\sum_{s<m \leq s^{\prime}} \rho_{m} \kappa_{+}(m)}{n_{u}(t)} \\
& \geq \kappa_{u,+}(s) \frac{v_{+}\left(s^{\prime}\right)-v_{+}(s)}{n_{u}(t)} \geq \frac{\alpha}{t^{2}} \text { with } \alpha=\left(c_{0}-1\right) / c_{0}^{2} .
\end{aligned}
$$

Consequently, with $\alpha^{\prime}=\left(c^{2} \alpha\right) /\left(2 c_{0}^{2}(1+2 c \alpha)\right)$ :

$$
\operatorname{Re}\left(1-f^{+}(u t)\right) \geq \frac{c}{n_{u}(t)} \frac{c \alpha}{1+2 c \alpha}=\frac{\alpha^{\prime}}{\left(\kappa_{u,+} \circ v_{+}^{-1}\right)^{-1}\left(1 / t^{2}\right)} .
$$

If now $m \longmapsto \kappa_{u,+}(m)$ is bounded, the previous inequality is valid as long as $\left(\kappa_{u,+} \circ v_{+}^{-1}\right)^{-1}\left(1 / t^{2}\right)$ is defined. For smaller $t,\left(\kappa_{u,+} \circ v_{+}^{-1}\right)^{-1}\left(1 / t^{2}\right)=+\infty$ and the previous lower-bound becomes trivial.

In the same way, with $\kappa_{u,-}(m)=\sum_{-m \leq k \leq l \leq-1} T_{k}^{l}(u)$, we have:

$$
\operatorname{Re}\left(1-f^{-}(u t)\right) \geq \alpha^{\prime} /\left(\kappa_{u,-} \circ v_{-}^{-1}\right)^{-1}\left(1 / t^{2}\right) .
$$

To prove a lower bound, we use (6.1), giving for some constant $c_{3}>0$ independent on $u$ :

$$
\operatorname{Re}\left(1-\chi_{D}(u t)\right) \geq p_{0}^{\prime} \operatorname{Re}\left(1-f^{+}(u t)\right)+q_{0}^{\prime} \operatorname{Re}\left(1-f^{-}(u t)\right)-c_{3} / F^{-1}(1 / t) .
$$

Recall that $G_{u,+}^{2}=F^{2}+\kappa_{u,+} \circ v_{+}^{-1}+\kappa_{u,-} \circ v_{-}^{-1}$. Then, for some constant $\beta>0$ independent on $u$ and $t$, we have:

$$
\beta \leq \frac{G_{u,+}^{-1}(1 / t)}{\min \left\{F^{-1}(1 / t),\left(\kappa_{u,+} \circ v_{+}^{-1}\right)^{-1}\left(1 / t^{2}\right),\left(\kappa_{u,-} \circ v_{-}^{-1}\right)^{-1}\left(1 / t^{2}\right)\right\}} \leq 1 .
$$

Fix $t>0$ and suppose for example that $\left(\kappa_{u,+} \circ v_{+}^{-1}\right)^{-1}\left(1 / t^{2}\right) \leq\left(\kappa_{u,-} \circ v_{-}^{-1}\right)^{-1}\left(1 / t^{2}\right)$. This leads to the following discussion:

- If $\left(1 / F^{-1}(1 / t)\right) \leq p_{0}^{\prime} \operatorname{Re}\left(1-f^{+}(u t)\right) /\left(2 c_{3}\right)$ and $F^{-1}(1 / t) \geq\left(\kappa_{u,+} \circ v_{+}^{-1}\right)^{-1}\left(1 / t^{2}\right)$, then:

$$
\operatorname{Re}\left(1-\chi_{D}(u t)\right) \geq\left(p_{0}^{\prime} / 2\right) \operatorname{Re}\left(1-f^{+}(u t)\right) \geq \frac{p_{0}^{\prime} \alpha^{\prime} / 2}{\left(\kappa_{u,+} \circ v_{+}^{-1}\right)^{-1}\left(1 / t^{2}\right)} \geq \frac{\beta p_{0}^{\prime} \alpha^{\prime} / 2}{G_{u,+}^{-1}(1 / t)} .
$$

- If $\left(1 / F^{-1}(1 / t)\right) \leq p_{0}^{\prime} \operatorname{Re}\left(1-f^{+}(u t)\right) /\left(2 c_{3}\right)$ and $F^{-1}(1 / t) \leq\left(\kappa_{u,+} \circ v_{+}^{-1}\right)^{-1}\left(1 / t^{2}\right)$, then, by lemma 6.5 and proposition 4.8, for absolute constants $c>0$ and $c^{\prime}>0$ :

$$
\operatorname{Re}\left(1-\chi_{D}(u t)\right) \geq c R(u t) \geq c^{\prime} / F^{-1}(1 / t) \geq \beta c^{\prime} / G_{u,+}^{-1}(1 / t) .
$$


- If $\left(1 / G_{u,+}^{-1}(1 / t)\right)>p_{0}^{\prime} \operatorname{Re}\left(1-f^{+}(u t)\right) /\left(2 c_{3}\right)$, then $F^{-1}(1 / t)<\left(2 c_{3} /\left(p_{0}^{\prime} \alpha^{\prime}\right)\right)\left(\kappa_{u,+} \circ\right.$ $\left.v_{+}^{-1}\right)^{-1}\left(1 / t^{2}\right)$. We obtain the inequality:

$$
\frac{\beta}{G_{u,+}^{-1}(1 / t)} \leq \frac{1}{F^{-1}(1 / t) \min \left\{p_{0}^{\prime} \alpha^{\prime} /\left(2 c_{3}\right), 1\right\}} .
$$

We conclude as in the previous case, via $\operatorname{Re}\left(1-\chi_{D}(u t)\right) \geq c R(u t) \geq c^{\prime} / F^{-1}(1 / t)$. This completes the proof of the proposition.

6.3. Preliminaries for estimating $\left|1-\chi_{D}(u t)\right|$. We shall prove a similar result for $\left|1-\chi_{D}(u t)\right|$, but the details are a little more delicate. We still fix $u \in S_{+}^{d-1}$ and $t>0$.

We use proposition 5.2 concerning $f^{+}$and its symmetric analogue for $f^{-}$. To precise the dependency with respect to $f^{+}$or $f^{-}$, we put a superscript $(+$or -$)$ on $A_{n}, B_{n}$, etc. For example:

$$
f^{+}(u t)=\lim _{n \rightarrow+\infty} A_{n}^{+}(u t) / B_{n}^{+}(u t) .
$$

Keeping the same sets of summation, the expressions corresponding to $K_{r}^{u,-}(n)$, $L_{r}^{u,-}(n)$, etc, are deduced from $K_{r}^{u,+}(n), L_{r}^{u,+}(n)$ by replacing in proposition 5.2 all $\left(q_{k}, p_{k}\right)$ by $\left(p_{-k}, q_{-k}\right)$. Any $\rho_{k}$ becomes $\rho_{-k-1} q_{0} / p_{0}$. A very important point is that $T_{k}^{l}(u)$ is simply transformed into $T_{-l}^{-k}(u)$.

Let us begin with a formal computation on reversed continued fractions.

Lemma 6.8. Let $n \geq 1$ and consider the formal reduced continued fraction:

$$
\frac{U_{n}}{V_{n}}=\left[\left(-c_{1}, d_{1}\right) ;\left(-c_{2}, d_{2}\right) ; \cdots ;\left(-c_{n}, d_{n}\right)\right] .
$$

Consider the reduced reversed continued fraction:

$$
\frac{\tilde{U}_{n}}{\tilde{V}_{n}}=\left[\left(-1 / c_{n}, d_{n} / c_{n}\right) ;\left(-1 / c_{n-1}, d_{n-1} / c_{n-1}\right) ; \cdots ;\left(-1 / c_{1}, d_{1} / c_{1}\right)\right] .
$$

Then we have the relation $V_{n}=c_{1} \cdots c_{n} \tilde{V}_{n}$.

Proof: Rereading if necessary section 3.1, we have:

$$
V_{n}=\left\langle e_{1},\left(\begin{array}{cc}
d_{n} & -c_{n} \\
1 & 0
\end{array}\right) \cdots\left(\begin{array}{cc}
d_{1} & -c_{1} \\
1 & 0
\end{array}\right) e_{1}\right\rangle
$$

Transposing and next conjugating the matrices with diag $(1,-1)$, which preserve the first vector $e_{1}$ of the canonical basis:

$$
\begin{aligned}
V_{n} & =\left\langle e_{1},\left(\begin{array}{cc}
d_{1} & 1 \\
-c_{1} & 0
\end{array}\right) \cdots\left(\begin{array}{cc}
d_{n} & 1 \\
-c_{n} & 0
\end{array}\right) e_{1}\right\rangle \\
& =\left\langle e_{1},\left(\begin{array}{cc}
d_{1} & -1 \\
c_{1} & 0
\end{array}\right) \cdots\left(\begin{array}{cc}
d_{n} & -1 \\
c_{n} & 0
\end{array}\right) e_{1}\right\rangle \\
& =c_{1} \cdots c_{n}\left\langle e_{1},\left(\begin{array}{cc}
d_{1} / c_{1} & -1 / c_{1} \\
1 & 0
\end{array}\right) \cdots\left(\begin{array}{cc}
d_{n} / c_{n} & -1 / c_{n} \\
1 & 0
\end{array}\right) e_{1}\right\rangle .
\end{aligned}
$$

Hence $V_{n}=c_{1} \cdots c_{n} \tilde{V}_{n}$. This proves the lemma. 
Let us start from relation (4.6):

$$
\chi_{D}(u t)=\varphi_{0}(u t)\left(p_{0}^{\prime} \chi_{D}^{+}(u t)+q_{0}^{\prime} \chi_{D}^{-}(u t)\right)=\left(\varphi_{0}(u t) / b_{0}\right)\left(\chi_{D}^{+}(u t)+a_{0} \chi_{D}^{-}(u t)\right) .
$$

This gives, using lemmas 4.8 and 6.5 and taking $t>0$ small, independently on $u$ :

$$
\begin{aligned}
\chi_{D}(u t)-1 & =\frac{\varphi_{0}(u t)}{b_{0}}\left(\chi_{D}^{+}(u t)+a_{0} \chi_{D}^{-}(u t)-b_{0} / \varphi_{0}(u t)\right) \\
& =\frac{\varphi_{0}(u t)}{b_{0}}\left(f^{+}(u t)+a_{0} f^{-}(u t)-b_{0} / \psi_{0}(u t)\right)+O(R(t)) \\
& =\frac{\varphi_{0}(u t)}{b_{0}}\left(\frac{A_{n}^{+}(u t)}{B_{n}^{+}(u t)}+a_{0} \frac{A_{m}^{-}(u t)}{B_{m}^{-}(u t)}-b_{0} / \psi_{0}(u t)\right) \\
& +\frac{\varphi_{0}(u t)}{b_{0}}\left(\sum_{k>n} \frac{\rho_{k}}{B_{k}^{+}(u t) B_{k-1}^{+}(u t)}+\sum_{k>m} \frac{a_{0}^{2} \rho_{-k-1}}{B_{k}^{-}(u t) B_{k-1}^{-}(u t)}\right)+O(R(t)),
\end{aligned}
$$

with $O($ ) uniform in $u$ and arbitrary $n \geq 1, m \geq 1$. As a result:

$$
\begin{aligned}
& \chi_{D}(u t)-1 \\
= & \frac{\varphi_{0}(u t)}{b_{0} B_{n}^{+}(u t) B_{m}^{-}(u t)}\left(A_{n}^{+}(u t) B_{m}^{-}(u t)+a_{0} A_{m}^{-}(u t) B_{n}^{+}(u t)\right. \\
& \left.-\left(b_{0} / \psi_{0}(u t)\right) B_{n}^{+}(u t) B_{m}^{-}(u t)\right)+\frac{\varphi_{0}(u t)}{b_{0}} R_{-m, n}(u t)+O(R(t)),
\end{aligned}
$$

with some error term $\left|R_{-m, n}(u t)\right| \leq\left(v_{+}(n) /\left|B_{n}^{+}(u t)\right|^{2}\right)+a_{0}\left(v_{-}(m) /\left|B_{m}^{-}(u t)\right|^{2}\right)$, by lemma 3.1, and also $O\left(\right.$ ) uniform in $u \in S_{+}^{d-1}$.

Lemma 6.9. Let $n \geq 1, m \geq 1$ and consider the following reduced continued fraction:

$$
\begin{aligned}
& \frac{\tilde{A}_{m+n+1}(u t)}{\tilde{B}_{m+n+1}(u t)}= \\
& =\left[\left(-a_{-m}, b_{-m} / \psi_{-m}(u t)\right) ;\left(-a_{-m+1}, b_{-m+1} / \psi_{-m+1}(u t)\right) ; \cdots ;\left(-a_{n}, b_{n} / \psi_{n}(u t)\right)\right] .
\end{aligned}
$$

Then we have the following expression:

$$
\begin{aligned}
& \tilde{B}_{m+n+1}(u t)= \\
& -a_{-1} \cdots a_{-m}\left(A_{n}^{+}(u t) B_{m}^{-}(u t)+a_{0} A_{m}^{-}(u t) B_{n}^{+}(u t)-\left(b_{0} / \psi_{0}(u t)\right) B_{n}^{+}(u t) B_{m}^{-}(u t)\right) .
\end{aligned}
$$

Proof: Fix $m \geq 1$. We now observe that the two functions:

$$
\begin{gathered}
n \longmapsto-\tilde{B}_{m+n+1}(u t) /\left(a_{-1} \cdots a_{-m}\right) \text { and } \\
n \longmapsto A_{n}^{+}(u t) B_{m}^{-}(u t)+a_{0} A_{m}^{-}(u t) B_{n}^{+}(u t)-\left(b_{0} / \psi_{0}(u t)\right) B_{n}^{+}(u t) B_{m}^{-}(u t)
\end{gathered}
$$

check the same recursive relation $X_{n}=\left(b_{n} / \psi_{n}(u t)\right) X_{n-1}-a_{n} X_{n-2}$, for $n \geq 1$. We just verify that they coincide for the values $n=0$ and $n=1$.

First of all, $\tilde{B}_{m}(u t) /\left(a_{-1} \cdots a_{-m}\right)=B_{m}^{-}(u t)$ and:

$$
A_{m}^{-}(u t)=\left(1 / a_{-1}\right) \theta^{-1} B_{m-1}^{-}(u t)=\tilde{B}_{m-1}(u t) /\left(a_{-1} \cdots a_{-m}\right),
$$

by lemma 6.8 . For $n=0$ we have $-\tilde{B}_{m+1}(u t) /\left(a_{-1} \cdots a_{-m}\right)$ and $a_{0} A_{m}^{-}(u t)-$ $\left(b_{0} / \psi_{0}(u t)\right) B_{m}^{-}(u t)$. Since one has:

$$
\tilde{B}_{m+1}(u t)=\left(b_{0} / \psi_{0}(u t)\right) \tilde{B}_{m}(u t)-a_{0} \tilde{B}_{m-1}(u t),
$$


this gives the result for $n=0$. Concerning $n=1$, we have:

$$
\begin{aligned}
\tilde{B}_{m+2}(u t) & =\frac{b_{1}}{\psi_{1}(u t)} \tilde{B}_{m+1}(u t)-a_{1} \tilde{B}_{m}(u t) \\
& =\left(\frac{b_{1}}{\psi_{1}(u t)} \frac{b_{0}}{\psi_{0}(u t)}-a_{1}\right) \tilde{B}_{m}(u t)-\frac{b_{1}}{\psi_{1}(u t)} a_{0} \tilde{B}_{m-1}(u t) .
\end{aligned}
$$

This has to be compared with $a_{1} B_{m}^{-}(u t)+a_{0}\left(b_{1} / \psi_{1}(u t)\right) A_{m}^{-}(u t)$ $-\left(b_{0} / \psi_{0}(u t)\right)\left(b_{1} / \psi_{1}(u t)\right) B_{m}^{-}(u t)$. As this is equal, the conclusion of the lemma now follows.

As a consequence of this lemma, we obtain:

$$
\chi_{D}(u t)-1=-\frac{\varphi_{0}(u t)}{b_{0}}\left(a_{0} \rho_{-m-1} \frac{\tilde{B}_{m+n+1}(u t)}{B_{n}^{+}(u t) B_{m}^{-}(u t)}-R_{-m, n}(u t)\right)+O(R(t)) .
$$

It now follows from proposition 5.2 that:

$$
\left|B_{n}^{+}(u t)\right|^{2}=\sum_{r=0}^{n} t^{2 r} L_{r}^{u,+}(n)
$$

with $H_{r}\left(\left(k_{i}\right),\left(l_{j}\right)\right):=\#\left\{0 \leq i \leq r \mid l_{i}+1<k_{i+1}\right\}$ and:

$$
L_{r}^{u,+}(n)=\sum_{0 \leq l_{0}<k_{1} \leq l_{1}<\cdots<k_{r} \leq l_{r}<k_{r+1} \leq n+1} \rho_{l_{0}} T_{k_{1}}^{l_{1}}(u) \cdots T_{k_{r}}^{l_{r}}(u) \rho_{k_{r+1}-1} 2^{H_{r}\left(\left(k_{i}\right),\left(l_{j}\right)\right)} .
$$

As a result, setting $W_{-m, n}(u t)=a_{0} \rho_{-m-1} \tilde{B}_{m+n+1}(u t)$, we have:

$$
\left|W_{-m, n}(u t)\right|^{2}=\sum_{0 \leq r \leq n+m+1} t^{2 r} U_{r}(u),
$$

with $H_{r}\left(\left(k_{i}\right),\left(l_{i}\right)\right)=\#\left\{0 \leq i \leq r \mid l_{i}+1<k_{i+1}\right\}$ and:

$$
\begin{aligned}
U_{r}(u)= & a_{0}^{2} \rho_{-m-1}^{2} \sum_{-m-1 \leq l_{0}<k_{1} \leq l_{1}<\cdots<k_{s} \leq l_{s}<k_{s+1} \leq n+1} \theta^{-m-1} \rho_{l_{0}+m+1} T_{k_{1}}^{l_{1}}(u) \cdots T_{k_{s}}^{l_{s}}(u) \theta^{-m-1} \\
& \times \rho_{k_{s+1}-1+m+1} 2^{H_{s}\left(\left(k_{i}\right),\left(l_{i}\right)\right)} .
\end{aligned}
$$

After a cocycle simplification we arrive at:

$$
U_{r}(u)=a_{0}^{2} \sum_{-m-1 \leq l_{0}<k_{1} \leq l_{1}<\cdots<k_{s} \leq l_{s}<k_{s+1} \leq n+1} \rho_{l_{0}} T_{k_{1}}^{l_{1}}(u) \cdots T_{k_{s}}^{l_{s}}(u) \rho_{k_{s+1}-1} 2^{H_{s}\left(\left(k_{i}\right),\left(l_{i}\right)\right)} .
$$

6.4. Order of $\left|1-\chi_{D}(u t)\right|$. Our aim in this section is to show the following result.

Proposition 6.10. There is a constant $C \geq 1$ so that for $t>0$ small enough, uniformly in $u \in S_{+}^{d-1}$ :

$$
\frac{1}{C} \leq G_{u}^{-1}(1 / t)\left|1-\chi_{D}(u t)\right| \leq C .
$$

Proof: Let us start from (6.5). Set:

$$
\mathcal{F}(u t)=\frac{W_{-m, n}(u t)}{B_{n}^{+}(u t) B_{m}^{-}(u t)}-R_{-m, n}(u t) .
$$

Recall that $\mathcal{F}(u t)$ is independent from $m \geq 1$ and $n \geq 1$, see (6.5). We then have:

$$
\chi_{D}(u t)-1=-\left(\varphi_{0}(u t) / b_{0}\right) \mathcal{F}(u t)+O(R(t))
$$


where the last term is uniform in $u \in S_{+}^{d-1}$. The integers $m \geq 1, n \geq 1$ are arbitrary for the moment. Using the upper-bound on $R_{-m, n}(u t)$ recalled in the last section, consequence of lemma 3.1, we get:

$$
\begin{aligned}
& |\mathcal{F}(u t)| \geq \frac{\left|W_{-m, n}(u t)\right|}{\left|B_{n}^{+}(u t)\right|\left|B_{m}^{-}(u t)\right|}-\frac{v_{+}(n)}{\left|B_{n}^{+}(u t)\right|^{2}}-a_{0} \frac{v_{-}(m)}{\left|B_{m}^{-}(u t)\right|^{2}} \\
& \geq \frac{1}{\left|B_{n}^{+}(u t)\right|\left|B_{m}^{-}(u t)\right|}\left(\left|W_{-m, n}(u t)\right|-v_{+}(n) \frac{\left|B_{m}^{-}(u t)\right|}{\left|B_{n}^{+}(u t)\right|}-a_{0} v_{-}(m) \frac{\left|B_{n}^{+}(u t)\right|}{\left|B_{m}^{-}(u t)\right|}\right) .
\end{aligned}
$$

Recall that $W_{-m, n}^{2}(u t)=\sum_{0 \leq r \leq n+m+1} t^{2 r} U_{r}(u)$, with $U_{s}(u)$ given by (6.6). In particular:

$$
U_{0}(u)=a_{0}^{2}\left(\sum_{-m-1 \leq l \leq n} \rho_{l}\right)^{2}=\left(v_{-}(m)+a_{0} v_{+}(n)\right)^{2} .
$$

Introduce $Z_{-m, n}^{2}(t)$ such that:

$$
\left|W_{-m, n}(u t)\right|^{2}-\left(a_{0}^{2}\left|B_{n}^{+}(u t)\right|^{2}+\left|B_{m}^{-}(u t)\right|^{2}\right)-2 a_{0} v_{+}(n) v_{-}(m)=Z_{-m, n}^{2}(u t) .
$$

Then $Z_{-m, n}^{2}(u t)=\sum_{1 \leq s \leq n+m+1} t^{2 s} V_{s}(u)$, where:

$$
V_{s}(u)=a_{0}^{2} \sum_{-m-1 \leq l_{0}<k_{1} \leq l_{1}<\cdots<k_{s} \leq l_{s}<k_{s+1} \leq n+1}^{\substack{l_{0}<0<k_{s+1} \\ l_{l_{1}}}} T_{k_{1}}^{l_{1}}(u) \cdots T_{k_{s}}^{l_{s}}(u) \rho_{k_{s+1}-1} 2^{H_{s}\left(\left(k_{i}\right),\left(l_{i}\right)\right)} .
$$

Observe now that:

$$
\begin{aligned}
& \left(v_{+}(n) \frac{\left|B_{m}^{-}(u t)\right|}{\left|B_{n}^{+}(u t)\right|}+a_{0} v_{-}(m) \frac{\left|B_{n}^{+}(u t)\right|}{\left|B_{m}^{-}(u t)\right|}\right)^{2} \\
= & v_{+}^{2}(n) \frac{\left|B_{m}^{-}(u t)\right|^{2}}{\left|B_{n}^{+}(u t)\right|^{2}}+a_{0}^{2} v_{-}^{2}(m) \frac{\left|B_{n}^{+}(u t)\right|^{2}}{\left|B_{m}^{-}(u t)\right|^{2}}+2 a_{0} v_{+}(n) v_{-}(m) \\
\leq & \left|B_{m}^{-}(u t)\right|^{2}+a_{0}^{2}\left|B_{n}^{+}(u t)\right|^{2}+2 a_{0} v_{+}(n) v_{-}(m) \\
\leq & \left|W_{-m, n}(u t)\right|^{2}-Z_{-m, n}^{2}(u t) \leq\left|W_{-m, n}(u t)\right|^{2} .
\end{aligned}
$$

This allows to write:

$$
\begin{aligned}
|\mathcal{F}(u t)| & \geq \frac{1}{\left|B_{n}^{+}(u t)\right|\left|B_{m}^{-}(u t)\right|}\left(\left|W_{-m, n}(u t)\right|-v_{+}(n) \frac{\left|B_{m}^{-}(u t)\right|}{\left|B_{n}^{+}(u t)\right|}-a_{0} v_{-}(m) \frac{\left|B_{n}^{+}(u t)\right|}{\left|B_{m}^{-}(u t)\right|}\right) \\
& \geq \frac{\left|W_{-m, n}(u t)\right|^{2}-\left(v_{+}(n) \frac{\left|B_{m}^{-}(u t)\right|}{\left|B_{n}^{+}(u t)\right|}+a_{0} v_{-}(m) \frac{\left|B_{n}^{+}(u t)\right|}{\left|B_{m}^{-}(u t)\right|}\right)^{2}}{2\left|W_{-m, n}(u t)\right|\left|B_{n}^{+}(u t)\right|\left|B_{m}^{-}(u t)\right|} \\
& \geq \frac{Z_{-m, n}^{2}(u t)}{2\left|W_{-m, n}(u t)\right|\left|B_{n}^{+}(u t)\right|\left|B_{m}^{-}(u t)\right|} .
\end{aligned}
$$

We now give upper-bounds on $\left|W_{-m, n}(u t)\right|$ and $\left|B_{n}^{+}(u t)\right|\left|B_{m}^{-}(u t)\right|$. Observe first that $L_{r}^{u,+}(n) \leq V_{r}(u) v_{+}(n) /\left(a_{0} v_{-}(m)\right)$, for $r \geq 1$, so that:

$$
\left|B_{n}^{+}(u t)\right|^{2}-v_{+}(n)^{2} \leq \frac{v_{+}(n)}{a_{0} v_{-}(m)} .
$$


Similarly, $\left|B_{m}^{-}(u t)\right|^{2}-v_{-}(m)^{2} \leq Z_{-m, n}^{2}(u t) v_{-}(m) /\left(a_{0} v_{+}(n)\right)$. We obtain:

$$
\begin{aligned}
& \left|W_{-m, n}(u t)\right|^{2} \\
& =\left(v_{-}(m)+a_{0} v_{+}(n)\right)^{2}+a_{0}^{2}\left(\left|B_{n}^{+}(u t)\right|^{2}-v_{+}(n)^{2}\right)+\left(\left|B_{m}^{-}(u t)\right|^{2}-v_{-}(m)^{2}\right)+Z_{-m, n}^{2}(u t) \\
& \leq\left(v_{-}(m)+a_{0} v_{+}(n)\right)^{2}+\left(a_{0} v_{+}(n) / v_{-}(m)+v_{-}(m) /\left(a_{0} v_{+}(n)\right)+1\right) Z_{-m, n}^{2}(u t) \\
& \leq\left(v_{-}(m)+a_{0} v_{+}(n)\right)^{2}\left[1+\frac{1}{a_{0} v_{-}(m) v_{+}(n)} Z_{-m, n}^{2}(u t)\right] .
\end{aligned}
$$

In the same way:

$$
\begin{aligned}
& \left|B_{n}^{+}(u t)\right|^{2}\left|B_{m}^{-}(u t)\right|^{2} \\
= & v_{+}(n)^{2} v_{-}(m)^{2}\left(1+\sum_{1 \leq r \leq n} t^{2 r} \frac{L_{r}^{u,+}(n)}{v_{+}(n)^{2}}\right)\left(1+\sum_{1 \leq r \leq m} t^{2 r} \frac{L_{r}^{u,-}(n)}{v_{-}(m)^{2}}\right) \\
= & v_{+}(n)^{2} v_{-}(m)^{2}\left(1+\sum_{1 \leq s \leq m+n} t^{2 s} \sum_{0 \leq r \leq s} \frac{L_{r}^{u,+}(n) L_{s-r}^{u,-}(m)}{v_{+}(n)^{2} v_{-}(m)^{2}}\right) .
\end{aligned}
$$

Notice that $\sum_{0 \leq r \leq s} L_{r}^{u,+}(n) L_{s-r}^{u,-}(m) \leq V_{s}(u) v_{+}(n) v_{-}(m) / a_{0}$. Therefore:

$$
\left|B_{n}^{+}(u t)\right|^{2}\left|B_{m}^{-}(u t)\right|^{2} \leq v_{+}(n)^{2} v_{-}(m)^{2}\left(1+\frac{Z_{-m, n}^{2}(u t)}{a_{0} v_{+}(n) v_{-}(m)}\right)
$$

Inserting these two upper-bounds in (6.9) and using in the last step that the function $x \longmapsto x /(1+x)$ is increasing, we obtain:

$$
\begin{aligned}
|\mathcal{F}(u t)| & \geq \frac{1}{2\left(v_{-}(m) / a_{0}+v_{+}(n)\right)} \frac{Z_{-m, n}^{2}(u t) /\left(a_{0} v_{+}(n) v_{-}(m)\right)}{1+Z_{-m, n}^{2}(u t) /\left(a_{0} v_{+}(n) v_{-}(m)\right)} \\
& \geq \frac{1}{2\left(v_{-}(m) / a_{0}+v_{+}(n)\right)} \frac{t^{2} V_{1}(u) /\left(a_{0} v_{+}(n) v_{-}(m)\right)}{1+t^{2} V_{1}(u) /\left(a_{0} v_{+}(n) v_{-}(m)\right)} .
\end{aligned}
$$

Let us now focus on $V_{1}(u)$ that we write $V_{1}(u)=V_{u, 1}(-m, n)$. Set $\kappa_{u}(r, s)=$ $\sum_{r \leq k \leq l \leq s} T_{k}^{l}(u)$, for $r \leq s$. We assume first that $\sum_{-\infty<k \leq l<+\infty} T_{k}^{l}(u)=+\infty$. We obtain:

$$
\begin{aligned}
V_{u, 1}(-m, n) & =a_{0}^{2} \sum_{-m-1 \leq l_{0}<k_{1} \leq l_{1}<k_{2} \leq n+1} \rho_{l_{0}} T_{k_{1}}^{l_{1}}(u) \rho_{k_{2}-1} 2^{H_{1}\left(\left(k_{i}\right),\left(l_{i}\right)\right)} \\
& \geq a_{0}^{2} \sum_{-m \leq l_{0} \leq 0 \leq k_{2} \leq n} \rho_{l_{0}-1} \rho_{k_{2}} \kappa_{u}\left(l_{0}, k_{2}\right) .
\end{aligned}
$$

We next have the existence of a constant $c>0$ independent on $u \in S_{+}^{d-1}$ so that for all $n \geq 1$ :

$$
|\mathcal{F}(u t)| \geq \frac{c}{n} \frac{\left(c t^{2} / n^{2}\right) V_{u, 1}\left(-v_{-}^{-1}(n), v_{+}^{-1}(n)\right)}{1+\left(c t^{2} / n^{2}\right) V_{u, 1}\left(-v_{-}^{-1}(n), v_{+}^{-1}(n)\right)} .
$$

Let $c_{0} \geq 2$ be such that for all $n \geq 0, v_{+}(n+1) \leq c_{0} v_{+}(n)$ and $v_{-}(n+1) \leq c_{0} v_{-}(n)$. Taking $t>0$, set $m_{u}(t)=\kappa_{u}\left(-v_{-}^{-1}(.), v_{+}^{-1}(.)\right)^{-1}\left(1 / t^{2}\right)$ As $\sup _{v \in S_{+}^{d-1}}\left\{\kappa_{u}\left(-v_{-}^{-1}(1), v_{+}^{-1}(1)\right)\right\}$ is clearly finite, we have $m_{u}(t) \geq 1$ for small enough $t$, uniformly in $u \in S_{+}^{d-1}$. Choose next $n_{u}(t)=c_{0}^{2}\left(m_{u}(t)+1\right)$. Let 
$r=v_{+}^{-1}\left(m_{u}(t)+1\right), s=v_{-}^{-1}\left(m_{u}(t)+1\right)$ and $r^{\prime}=v_{+}^{-1}\left(n_{u}(t)\right), s^{\prime}=v_{-}^{-1}\left(n_{u}(t)\right)$. This gives:

$$
\begin{aligned}
& v_{+}(r) \leq m_{u}(t)+1<v_{+}(r+1) \leq c_{0} v_{+}(r) \text { and } \\
& v_{+}\left(r^{\prime}\right) \leq c_{0}^{2}\left(m_{u}(t)+1\right)<v_{+}\left(r^{\prime}+1\right) \leq c_{0} v_{+}\left(r^{\prime}\right) .
\end{aligned}
$$

As a result, $c_{0}^{2}\left(m_{u}(t)+1\right) \geq v_{+}\left(r^{\prime}\right)-v_{+}(r) \geq\left(c_{0}-1\right)\left(m_{u}(t)+1\right)$ and $\left(m_{u}(t)+1\right) \geq$ $v_{+}(r) \geq\left(m_{u}(t)+1\right) / c_{0}$. In the same way, we have:

$$
\begin{aligned}
& v_{-}(s) \leq m_{u}(t)+1<v_{-}(s+1) \leq c_{0} v_{-}(s) \text { and } \\
& v_{-}\left(s^{\prime}\right) \leq c_{0}^{2}\left(m_{u}(t)+1\right)<v_{+}\left(s^{\prime}+1\right) \leq c_{0} v_{+}\left(s^{\prime}\right) .
\end{aligned}
$$

Also, $c_{0}^{2}\left(m_{u}(t)+1\right) \geq v_{-}\left(s^{\prime}\right)-v_{-}(s) \geq\left(c_{0}-1\right)\left(m_{u}(t)+1\right)$ and $\left(m_{u}(t)+1\right) \geq$ $v_{-}(s) \geq\left(m_{u}(t)+1\right) / c_{0}$.

We obtain:

$$
\begin{aligned}
\frac{V_{u, 1}\left(-v_{-}^{-1}\left(n_{u}(t)\right), v_{+}^{-1}\left(n_{u}(t)\right)\right)}{n_{u}(t)^{2}} & \geq a_{0}^{2} \frac{\sum_{r<l \leq r^{\prime}, s<k \leq s^{\prime}} \rho_{-k-1} \rho_{l} \kappa_{u}(-k, l)}{n_{u}(t)^{2}} \\
& \geq a_{0} \kappa_{u}(-s, r) \frac{\left(v_{+}\left(r^{\prime}\right)-v_{+}(r)\right)\left(v_{-}\left(s^{\prime}\right)-v_{-}(s)\right)}{n_{u}(t)^{2}} \\
& \geq \frac{a_{0}\left(c_{0}-1\right)^{2}\left(m_{u}(t)+1\right)^{2}}{t^{2} n_{u}(t)^{2}}=\frac{\alpha}{t^{2}},
\end{aligned}
$$

where $\alpha=a_{0}\left(c_{0}-1\right)^{2} / c_{0}^{2}$. At this point, the conclusion is that there is a constant $c^{\prime}>0$ so that for small enough $t>0$, uniformly in $u \in S_{+}^{d-1}$ :

$$
|\mathcal{F}(u t)| \geq \frac{c^{\prime}}{\kappa_{u}\left(-v_{-}^{-1}(.), v_{+}^{-1}(.)\right)^{-1}\left(1 / t^{2}\right)} .
$$

When $\sum_{-\infty<k \leq l<+\infty} T_{k}^{l}$ is bounded, the inequality is verified, as $\kappa_{u}\left(v_{-}^{-1}(.), v_{+}^{-1}(.)\right)^{-1}\left(1 / t^{2}\right)=+\infty$, for small enough $t>0$. The previous lowerbound is then obvious in that case.

In order to draw the conclusion, recall that $G_{u}^{2}(n)=F^{2}(n)+\kappa_{u}\left(-v_{-}^{-1}(n), v_{+}^{-1}(n)\right)$ and $1-\chi_{D}(u t)=\left(\varphi_{0}(u t) / b_{0}\right) \mathcal{F}(u t)+O(R(t))$, with $O()$ uniform in $u \in S_{+}^{d-1}$. Also, by lemma 6.5 :

$$
\left|1-\chi_{D}(u t)\right| \geq \operatorname{Re}\left(1-\chi_{D}(u t)\right) \geq c_{1} R(u t),
$$

for some absolute constant $c_{1}>0$. Similarly, for constants $c_{2}>0$ and $c_{3}>0$, we have the inequalities $c_{2} \leq R(t) F^{-1}(1 / t) \leq c_{3}$. Then, for constants $\beta>0$ and $c_{4}>0$ independent on $u \in S_{+}^{d-1}$, for small $t>0$ :

$$
\beta \leq \frac{G_{u}^{-1}(1 / t)}{\min \left\{F^{-1}(1 / t), \kappa_{u}\left(-v_{-}^{-1}(.), v_{+}^{-1}(.)\right)^{-1}\left(1 / t^{2}\right)\right\}}
$$

and:

$\left|1-\chi_{D}(u t)\right| \geq \frac{1}{2 b_{0}}|\mathcal{F}(u t)|-c_{4} R(t) \geq \frac{c^{\prime}}{2 b_{0} \kappa_{u}\left(-v_{-}^{-1}(.), v_{+}^{-1}(.)\right)^{-1}\left(1 / t^{2}\right)}-\frac{c_{4} c_{3}}{F^{-1}(1 / t)}$.

Fixing $t>0$, we then have the following discussion:

- If $c^{\prime} /\left(2 b_{0} \kappa_{u}\left(-v_{-}^{-1}(.), v_{+}^{-1}(.)\right)^{-1}\left(1 / t^{2}\right)\right) \quad \geq 2 c_{3} c_{4} / F^{-1}(1 / t) \quad$ and $\kappa_{u}\left(-v_{-}^{-1}(.), v_{+}^{-1}(.)\right)^{-1}\left(1 / t^{2}\right) \leq F^{-1}(1 / t)$ :

$$
\left|1-\chi_{D}(u t)\right| \geq \frac{c^{\prime}}{4 b_{0} \kappa_{u}\left(-v_{-}^{-1}(.), v_{+}^{-1}(.)\right)^{-1}\left(1 / t^{2}\right)} \geq \frac{c^{\prime} \beta}{4 b_{0} G_{u}^{-1}(1 / t)} .
$$


- If $c^{\prime} /\left(2 b_{0} \kappa_{u}\left(-v_{-}^{-1}(.), v_{+}^{-1}(.)\right)^{-1}\left(1 / t^{2}\right)\right) \quad \geq \quad 2 c_{3} c_{4} / F^{-1}(1 / t) \quad$ and $\kappa_{u}\left(-v_{-}^{-1}(.), v_{+}^{-1}(.)\right)^{-1}\left(1 / t^{2}\right)>F^{-1}(1 / t)$ :

$$
\left|1-\chi_{D}(u t)\right| \geq c_{1} c_{2} / \psi^{-1}(1 / t) \geq c_{1} c_{2} \beta / G_{u}^{-1}(1 / t) .
$$

- If $c^{\prime} /\left(2 b_{0} \kappa_{u}\left(-v_{-}^{-1}(.), v_{+}^{-1}(.)\right)^{-1}\left(1 / t^{2}\right)\right)<2 c_{3} c_{4} / F^{-1}(1 / t)$, then for some absolute constant $c_{5}>0$ (independent on $\left.u\right), 1 / F^{-1}(1 / t) \geq c_{5} / G_{u}^{-1}(1 / t)$. Then, as above:

$$
\left|1-\chi_{D}(u t)\right| \geq c_{1} c_{2} / F^{-1}(1 / t) \geq c_{1} c_{2} c_{5} G_{u}^{-1}(1 / t) .
$$

This completes the proof of the lower bound. We next turn to the proof of the upper-bound. Let us start from the following inequality, for any $m \geq 1, n \geq 1$, using lemma 3.1:

$$
\begin{aligned}
\left|1-\chi_{D}(u t)\right| & \leq \frac{1}{b_{0}}|\mathcal{F}(u t)|+O(R(t)) \\
& \leq \frac{\left|W_{-m, n}(u t)\right|}{b_{0}\left|B_{n}^{+}(u t)\right|\left|B_{m}^{-}(u t)\right|}+\frac{v_{+}(n)}{\left|B_{n}^{+}(u t)\right|^{2}}+\frac{a_{0} v_{-}(m)}{\left|B_{m}^{-}(u t)\right|^{2}}+O(R(t)),
\end{aligned}
$$

with $O\left(\right.$ ) uniform in $u \in S_{+}^{d-1}$. Observe that from the second line in (6.9):

$$
\begin{aligned}
\frac{v_{+}(n)}{\left|B_{n}^{+}(u t)\right|^{2}}+\frac{a_{0} v_{-}(m)}{\left|B_{m}^{-}(u t)\right|^{2}} & \leq \frac{1}{\left|B_{n}^{+}(u t)\right|\left|B_{m}^{-}(u t)\right|}\left(v_{+}(n) \frac{\left|B_{m}^{-}(u t)\right|}{\left|B_{n}^{+}(u t)\right|}+a_{0} v_{-}(m) \frac{\left|B_{n}^{+}(u t)\right|}{\left|B_{m}^{-}(u t)\right|}\right) \\
& \leq \frac{\left|W_{-m, n}(u t)\right|}{\left|B_{n}^{+}(u t)\right|\left|B_{n}^{-}(u t)\right|} .
\end{aligned}
$$

Since $R(t)=O\left(1 / F^{-1}(1 / t)\right)=O\left(1 / G_{u}^{-1}(1 / t)\right)$, uniformly on $u \in S_{+}^{d-1}$, there exists some absolute constant $C>0$ such that for small $t>0$ and all $m \geq 1$ and $n \geq 1$ :

$$
\left|1-\chi_{D}(u t)\right| \leq C \frac{\left|W_{-m, n}(u t)\right|}{\left|B_{n}^{+}(u t)\right|\left|B_{m}^{-}(u t)\right|}+\frac{C}{G_{u}^{-1}(1 / t)} .
$$

From (6.10) and lemma 3.1, we have:

$$
\frac{\left|W_{-m, n}(u t)\right|}{\left|B_{n}^{+}(u t)\right|\left|B_{m}^{-}(u t)\right|} \leq \frac{\left(v_{-}(m)+a_{0} v_{+}(n)\right) \sqrt{1+Z_{-m, n}^{2}(u t) /\left(a_{0} v_{-}(m) v_{+}(n)\right)}}{v_{+}(n) v_{-}(m)} .
$$

Let us recall that $Z_{-m, n}^{2}(u t)=\sum_{1 \leq s \leq m+n+1} t^{2 s} V_{s}(u)$, where $V_{s}(u)$ is given by relation (6.7), so checks $V_{s}(u) \leq a_{0} v_{-}(m) v_{+}(n) \kappa_{u}(-m, n)^{s}$, still setting $\kappa_{u}(-m, n)=$ $\sum_{-m \leq k \leq l \leq n} T_{k}^{l}(u)$. As a result, for another constant $C>0$ independent on $u \in S_{+}^{\bar{d}-\overline{1}}$, small $t>0$ and any $n \geq 1$ :

$$
\begin{aligned}
& \left|1-\chi_{D}(u t)\right| \leq \\
& \leq \frac{C}{n} \sqrt{1+\sum_{1 \leq s \leq v_{-}^{-1}(n)+v_{+}^{-1}(n)+1} t^{2 s}\left(\kappa_{u}\left(-v_{-}^{-1}(n), v_{+}^{-1}(n)\right)\right)^{s}}+\frac{C}{G_{u}^{-1}(1 / t)} \\
& \leq \frac{C}{n} \sqrt{1+\sum_{1 \leq s \leq v_{-}^{-1}(n)+v_{+}^{-1}(n)+1} t^{2 s} G_{u}^{2 s}(n)}+\frac{C}{G_{u}^{-1}(1 / t)} .
\end{aligned}
$$

Choose $n=G_{u}^{-1}(1 / 2 t)$. In particular, $G_{u}(n) \leq 1 /(2 t)$. This gives:

$$
\left|1-\chi_{D}(u t)\right| \leq \frac{C}{G_{u}^{-1}(1 /(2 t))} \sqrt{1+\sum_{s \geq 1}(1 / 2)^{2 s}}+\frac{C}{G_{u}^{-1}(1 / t)} .
$$


By lemma 6.2, there is a constant $C^{\prime}$ independent on $u \in S_{+}^{d-1}$ so that for small $t>0$ :

$$
\left|1-\chi_{D}(u t)\right| \leq \frac{C^{\prime}}{G_{u}^{-1}(1 / t)} .
$$

This concludes the proof of the proposition.

6.5. Conclusion. Let us now prove theorem 2.4. Recall that we use the notation $\asymp$ to denote the fact that two quantities are equal up to absolute multiplicative positive constants.

First of all, for $n \geq 1$ :

$$
\begin{aligned}
\sum_{-v_{-}^{-1}(n) \leq k \leq l \leq v_{+}^{-1}(n)} \rho_{k} \rho_{l}\left(\frac{1}{\rho_{k}^{2}}+\frac{1}{\rho_{l}^{2}}\right) & \asymp \sum_{-v_{-}^{-1}(n) \leq k \leq v_{+}^{-1}(n)} \rho_{k} \sum_{-v_{-}^{-1}(n) \leq k \leq v_{+}^{-1}(n)} 1 / \rho_{k} \\
& \asymp F^{2}(n) .
\end{aligned}
$$

From this it is immediate to deduce that $\Phi_{s t r}(n) \asymp F(n)$. In a similar way, one obtains $G_{u,+}(n) \asymp \Phi_{u,+}(n)$ and $G_{u}(n) \asymp \Phi_{u}(n)$.

By propositions 6.7, 6.10 and theorem 4.2, writing the integral as in (4.3) and using the fact that $\operatorname{Re}(1 /(1-z))=\operatorname{Re}(1-z) /|1-z|^{2}$, the random walk is recurrent if and only if:

$$
\int_{(u, t) \in S_{+}^{d-1} \times(0, \eta)} \frac{\left(\Phi_{u}^{-1}(1 / t)\right)^{2}}{\Phi_{u,+}^{-1}(1 / t)} t^{d-1} d u d t=+\infty, \text { for some } \eta>0 .
$$

Let $n_{0}$ be such that $1 / n_{0} \leq \eta$. Uniformly in $u \in S_{+}^{d-1}$, on each interval $[1 /(n+$ $1), 1 / n], n \geq n_{0}$ :

$$
\frac{\left(\Phi_{u}^{-1}(1 / t)\right)^{2}}{\Phi_{u,+}^{-1}(1 / t)} t^{d-1} \asymp \frac{\left(\Phi_{u}^{-1}(n)\right)^{2}}{\Phi_{u,+}^{-1}(n)} n^{-d+1},
$$

by lemma 6.2 . Cutting $(0, \eta)$ in the contiguous intervals $[1 /(n+1), 1 / n], n \geq n_{0}$, each one of length of order $1 / n^{2}$, the condition is equivalent to the one given in the statement of theorem 2.4.

Concerning proposition 2.5, we first show the following lemma:

Lemma 6.11. When there exists $C>0$ so that for all $n \geq 1$ and all $u \in S_{+}^{d-1}$, $\Phi_{u}(n) \leq C \Phi_{u,+}(n)$, the random walk is recurrent if and only if:

$$
\sum_{n \geq 1} \int_{u \in S_{+}^{d-1}}\left(\Phi_{u,+}(n)\right)^{-d} d u=+\infty .
$$

Proof: By lemma 6.2, $\Phi_{u}^{-1} \asymp \Phi_{u,+}^{-1}$, uniformly in $u \in S_{+}^{d-1}$. From (6.11), the criterion for recurrence can be written in the reduced form:

$$
\int_{S_{+}^{d-1} \times(0, \eta)} \Phi_{u,+}^{-1}(1 / t) t^{d-1} d u d t=+\infty .
$$

Let $n_{0}$ be so that $\phi_{s t r}\left(n_{0}\right) \geq 1 / \eta$. Recall that for all $u \in S_{+}^{d-1}, \Phi_{u,+}(n) \geq \Phi_{s t r}(n)$. Since $\Phi_{u,+}\left(n_{0}\right)$ is clearly bounded in $u \in S_{+}^{d-1}$, using also lemma 6.2, the divergence 
of the previous integral is equivalent to that of:

$$
\begin{aligned}
& \sum_{n \geq n_{0}} \int_{S_{+}^{d-1}} \int_{1 / \Phi_{u,+}(n+1)}^{1 / \Phi_{u,+}(n)} \Phi_{u,+}^{-1}(1 / t) t^{d-1} d t d u \\
& \asymp \sum_{n \geq n_{0}} n \int_{S_{+}^{d-1}} \int_{1 / \Phi_{u,+}(n+1)}^{1 / \Phi_{u,+}(n)} t^{d-1} d t d u \\
& \asymp \int_{S_{+}^{d-1}} \sum_{n \geq n_{0}} n\left(\frac{1}{\left(\Phi_{u,+}(n)\right)^{d}}-\frac{1}{\left(\Phi_{u,+}(n+1)\right)^{d}}\right) d u . \\
& \asymp \int_{S_{+}^{d-1}} \lim _{N \rightarrow+\infty} \sum_{n=n_{0}}^{N}\left(\frac{1}{\left(\Phi_{u,+}(n)\right)^{d}}-\frac{1}{\left(\Phi_{u,+}(N+1)\right)^{d}}\right) d u,
\end{aligned}
$$

using an Abel transform. Now if $\int_{S_{+}^{d-1}} \sum_{n \geq 1}\left(\Phi_{u,+}(n)\right)^{-d} d u<+\infty$, then the previous integral is finite. If it is infinite, then applying Fatou's lemma (as $N \rightarrow+\infty)$, the integral is also infinite.

Concerning proposition 2.5, we always have $\Phi_{u}^{-1} \leq \Phi_{u,+}^{-1}$, so a sufficient condition for showing the finiteness of the integral in (6.11) is to show that of (6.12). As proved above, this is also equivalent to the finiteness of the integral appearing in the statement of lemma 6.11.

Another sufficient condition comes from the remark that $\Phi_{u,+} \geq \Phi_{s t r}$, independent of $u$ in the compact set $S_{+}^{d-1}$. This gives the sufficient conditions stated for transience in the proposition, using that $\Phi_{s t r}(n)$ always has at least order $\sqrt{n}$ for the case $d \geq 3$. When $d=2$ and $\rho_{n}=1$, for all $n \in \mathbb{Z}$, then $\Phi_{\text {str }}(n)$ has order $n$.

When $m_{n}=0$ for all $n \in \mathbb{Z}$, then $\Phi_{u}=\Phi_{u,+}=\Phi_{\text {str }}$ and we can apply lemma 6.11. In the antisymmetric case, observe that:

$$
\begin{aligned}
\Phi_{u}^{2}(n) & =\Phi_{u,+}^{2}(n)+\sum_{-v_{-}^{-1}(n) \leq k \leq 0 \leq l \leq v_{+}^{-1}(n)} T_{k}^{l}(u) \\
& =\Phi_{u,+}^{2}(n)+2 \sum_{0 \leq k, l \leq v_{+}^{-1}(n)} T_{\min (k, l)+1}^{\max (k, l)}(u) \leq 4 \Phi_{u,+}^{2}(n) .
\end{aligned}
$$

We can next apply lemma 6.11, using finally that $\Phi_{u}(0, n) \leq \Phi_{u,+}(0) \leq 2 \Phi_{u}(0, n)$. This completes the proof of the proposition.

\section{Examples and remarks; interpretation of the criterion}

7.1. Flat case $\left(\rho_{n}=1, n \in \mathbb{Z}\right)$. For simple random walk in $\mathbb{Z}^{d+1}$, we can apply the second point of proposition 2.5, as $m_{n}=0$ for all $n \in \mathbb{Z}$. Then $\Phi_{\text {str }}(n) \asymp n$ and the random walk is recurrent if and only if $\sum_{n>1} n^{-d}=+\infty$, hence $d=1$, as expected.

Let us now complement with a remark the study made in Brémont (2016) for the Campanino-Petritis model when the orientations $\left(\varepsilon_{n}\right)_{n \in \mathbb{Z}}$ are quasi-periodic.

Proposition 7.1. Let $d=1$ and for $n \in \mathbb{Z}, p_{n}=q_{n}=r_{n}=1 / 3$ and $\mu_{n}=\delta_{\varepsilon_{n}}$ where $\varepsilon_{n}=1_{[0,1 / 2)}(n \alpha)-1_{[1 / 2,1)}(n \alpha)$, where $n \alpha$ is taken modulo 1 . Then for almost-every $\alpha$, the random walk is recurrent. 
Proof: Recurrence is shown in Brémont (2016), proposition 1.5, for any irrational $\alpha$ having a continued fraction expansion $\left[a_{1}, a_{2}, \cdots\right]$ verifying:

$$
\sum_{n \geq 1} \frac{\log \left(1+a_{n}\right)}{a_{1}+\cdots+a_{n}}=+\infty
$$

In fact, $\sum_{n>1} 1 /\left(a_{1}+\cdots+a_{n}\right)=+\infty$ already for almost-every $\alpha$. This is an old theorem of Khintchine (1935), consequence of the classical result that $\left(a_{1}+\cdots+\right.$ $\left.a_{n}\right) /(n \log n)$ converges in probability to $1 / \log 2$. The latter follows from a weak form of the exponential mixing for the Gauss map.

7.2. General case. Let us begin with a simple example.

Proposition 7.2. Let $d=1$. Let $\delta>0$ with for all $n \in \mathbb{Z}, \delta \leq p_{n} \leq 1 / 2-\delta$. Set $q_{n}=1 / 2-p_{n}, r_{n}=1 / 2$ and $\mu_{n}=\left(\delta_{1}+\delta_{-1}\right) / 2$. Suppose that $\rho_{n} \asymp|n|^{\alpha_{ \pm}}$, as $n \rightarrow \pm \infty$, for constants $\alpha_{ \pm} \in \mathbb{R}$.

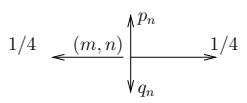

In this case, both coordinates are recurrent if and only if $\min \left\{\alpha_{-}, \alpha_{+}\right\} \geq-1$. The random walk is recurrent if and only if $\min \left\{\alpha_{-}, \alpha_{+}\right\} \geq 0$.

Proof: The first coordinate is always recurrent. We have $v_{+}(n) \asymp n^{1+\alpha_{+}}$or $\log n$ if $\alpha_{+}=-1$, idem for $v_{-}(n)$. The vertical coordinate is recurrent if and only if $v_{ \pm}(n) \rightarrow+\infty$, ie $\alpha_{ \pm} \geq-1$. Next, by the second point of proposition 2.5 , the random walk is recurrent if and only if $\sum_{n \geq 1}\left(1 / \Phi_{s t r}(n)\right)=+\infty$. Let $\alpha_{ \pm} \geq-1$, different from \pm 1 . We have $v_{+}(n) \asymp n^{1+\alpha_{+}}$and $w_{+}(n) \asymp n^{1-\alpha_{+}}$. Hence, $\Phi_{\text {str }}^{2}(n) \asymp$ $n^{1+\left(1-\alpha_{+}\right) /\left(1+\alpha_{+}\right)}+n^{1+\left(1-\alpha_{-}\right) /\left(1+\alpha_{-}\right)}$. Divergence holds for $\alpha_{ \pm} \geq 0$. The cases when $\alpha_{ \pm}$are +1 or -1 are treated in the same way.

Let us now turn towards an antisymmetric situation.

Proposition 7.3. Consider the antisymmetric case of proposition 2.5, point 3). Suppose that $m_{n}=c \neq 0$, for $n \geq 1$, and that $\rho_{n} \asymp n^{\alpha}$, as $n \rightarrow+\infty \geq 0$, where $\alpha \in \mathbb{R}$. Then:

- When $d=1$, the random walk is recurrent if and only if $\alpha \geq 1$.

- When $d=2$, the random walk is recurrent if and only if $\alpha \geq 3$.

Remark 7.4. When $d=1$ and in the particular antisymmetric case when $\mu_{n}=\delta_{1}$, $\mu_{-n}=\delta_{-1}$, for $n \geq 1$, and $\mu_{0}=1 / 2\left(\delta_{1}+\delta_{-1}\right)$, notice that horizontal steps are restricted to +1 in the Northern part and to -1 in the Southern part. Consequently the random walk can be either recurrent or transient, but necessarily its trajectory makes some spirals. In the flat case (giving $\alpha=0$ ), the random walk is transient. This confirms the remark made in Campanino and Petritis (2004) that these parameters lie deeply within the transience regime.

Proof: If $\alpha<-1$, the random walk is transient, as $\left(v_{+}(n)\right)$ is bounded. If $\alpha=-1$, then $w_{+}(n) \asymp n^{2}$ and $v_{+}(n) \asymp \ln n$. As a result, $\Phi_{s t r}^{2}(n) \geq n e^{c n}$, for some $c>0$, giving transience, by proposition 2.5 . 
- Supposing $-1<\alpha<1$, we show transience. First of all, $w_{+}(n) \asymp n^{1-\alpha}$ and $v_{+}(n) \asymp n^{1+\alpha}$.

$$
\begin{aligned}
\sum_{1 \leq k<l \leq n} \rho_{k} \rho_{l}\left(\sum_{k \leq s \leq l} 1 / \rho_{s}\right)^{2} & \asymp \int_{1 \leq x \leq y \leq n} x^{\alpha} y^{\alpha}\left(\int_{x}^{y} t^{-\alpha} d t\right)^{2} d x d y \\
& \asymp n^{2 \alpha+2} \int_{1 / n \leq x \leq y \leq 1} x^{\alpha} y^{\alpha}\left(\int_{n x}^{n y} t^{-\alpha} d t\right)^{2} d x d y \\
& \asymp n^{4} \int_{1 / n \leq x \leq y \leq 1} x^{\alpha} y^{\alpha}\left(\int_{x}^{y} t^{-\alpha} d t\right)^{2} d x d y \asymp n^{4} .
\end{aligned}
$$

As a result $\Phi_{u}^{2}(0, n) \asymp n^{1+(1-\alpha) /(1+\alpha)}+(c . u)^{2} n^{4 /(1+\alpha)}$, giving:

$$
\Phi_{u}(0, n) \asymp n^{1 /(1+\alpha)}+|c . u| n^{2 /(1+\alpha)} .
$$

When $d=1$, we have $|c . u|=|c|$, so $\Phi_{u}(0, n) \asymp n^{2 /(1+\alpha)}$. When $d \geq 2,\left(\Phi_{u}(0, n)\right)^{d} \geq$ $C n^{d /(1+\alpha)}$. The exponents are $>1$ in each case. The random walk is transient, by proposition 2.5 .

- Let now $\alpha>1$. Then $w_{+}(n) \asymp 1, v_{+}(n) \asymp n^{1+\alpha}$. If $d=1$, then $\Phi_{u}^{2}(0, n) \leq$ $C\left(n+(c, u)^{2} n^{2}\right)$, so $\Phi_{u}(0, n)=O(n)$ uniformly in $u$, so the random walk is recurrent, by proposition 2.5 . When $d=2$, consider first the expression:

$$
\begin{aligned}
& \left(\int_{1}^{n} x^{\alpha} d x\right) \int_{1}^{n} x^{\alpha}\left(\int_{x}^{+\infty} t^{-\alpha} d t\right)^{2} d x-\left(\int_{1}^{n} x^{\alpha} \int_{x}^{+\infty} t^{-\alpha} d t d x\right)^{2} \\
\asymp & \left(\int_{1}^{n} x^{\alpha} d x\right)\left(\int_{1}^{n} x^{2-\alpha}\right)-\left(\int_{1}^{n} x d x\right)^{2},
\end{aligned}
$$

since the integration coefficients are equal for each term on the right. When $1<\alpha<$ 3 , this term is equivalent to $1 /[(\alpha+1)(3-\alpha)]-1 / 4=(\alpha-1)^{2} /[(\alpha+1)(3-\alpha)] \neq 0$ times $n^{4}$. As a result:

$$
\begin{aligned}
\Phi_{u}^{2}(0, n) & \asymp n+(c . u)^{2} \int_{1 \leq x \leq y \leq n^{1 /(1+\alpha)}} x^{\alpha} y^{\alpha}\left(\int_{x}^{y} t^{-\alpha} d t\right)^{2} d x d y \\
& \asymp n+(c . u)^{2} n^{4 /(1+\alpha)} .
\end{aligned}
$$

In order to show transience, by proposition 2.5, we need to control the following quantity:

$$
\sum_{n \geq 1} \int_{u \in S_{+}^{1}} \frac{1}{\Phi_{u}^{2}(0, n)} \asymp \sum_{n \geq 1} \int_{0}^{\pi / 2} \frac{1}{n+\theta^{2} n^{4 /(1+\alpha)}} d \theta .
$$

Simply set $\theta=n^{1 / 2-2 /(1+\alpha)} x$, where $1 / 2-2 /(1+\alpha)<0$. It remains:

$$
\sum_{n \geq 1} \frac{1}{n} n^{1 / 2-2 /(1+\alpha)} \int_{0}^{(\pi / 2) n^{2 /(1+\alpha)-1 / 2}} \frac{1}{1+x^{2}} d x \asymp \sum_{n \geq 1} \frac{1}{n^{1 / 2+2 /(1+\alpha)}}<+\infty,
$$

as $1 / 2+2 /(1+\alpha)=(5+\alpha) /(2+2 \alpha)>1$. If $\alpha=3$, then $\Phi_{u}^{2}(0, n) \asymp n+(c . u)^{2} n \ln n \leq$ $C n \ln n$, uniformly in $u$. When $\alpha>3, \Phi_{u}^{2}(0, n) \asymp n+(c . u)^{2} n \leq C n$, uniformly in $u$. In any case $\sum_{1 \geq 1}\left(1 / \Phi_{u}^{2}(0, n)\right)=+\infty$, giving recurrence.

- If finally $\alpha=1$, then $w_{+}(n) \asymp \ln n, v_{+}(n) \asymp n^{2}$. When $d=1, \Phi_{u}^{2}(0, n) \leq$ $C\left(n \ln n+n^{2}(\ln n)^{2}\right)$, giving $\Phi_{u}(0, n)=O(n \ln n)$, uniformly in $u$, and the random 
walk is recurrent. When $d=2$ :

$$
\Phi_{u}^{2}(0, n) \geq K\left(n \ln n+(c . u)^{2} n^{2}\right), \text { for some } K>0 .
$$

In order to show transience, we just need to prove the finiteness of:

$$
\sum_{n \geq 1} \int_{0}^{\pi / 2} \frac{1}{n \ln n+\theta^{2} n^{2}} d \theta=\sum_{n \geq 1} \frac{1}{n \ln n} \int_{0}^{(\pi / 2) \sqrt{n / \ln n}} \frac{d x}{1+x^{2}} \sqrt{(\ln n) / n}<+\infty .
$$

This completes the proof of the proposition.

7.3. The half-pipe. Contrary to the flat case $\left(p_{n}=q_{n}, n \in \mathbb{Z}\right)$, one can somehow "suppress" the vertical dimension for some values of the parameters, indeed when $\sum_{n \in \mathbb{Z}}\left(1 / \rho_{n}\right)<+\infty$. In this case the environment strongly pushes towards $\mathbb{Z}^{d}$ and the vertical component is positive recurrent (this is a kind of random walk in a half-pipe when $d=1$ ). The random walk is then "essentially" $d$-dimensional. This explains the critical values of $d$ appearing in proposition 2.5 and that the random walk, in $\mathbb{Z}^{d+1}$, can be recurrent when $d=2$.

Proposition 7.5. Suppose that $\sum_{n \in \mathbb{Z}} 1 / \rho_{n}<+\infty$. In this situation:

- If $\sum_{n \in \mathbb{Z}} \frac{r_{n} m_{n}}{p_{n} \rho_{n}} \neq 0$, then the random walk is transient.

- If $d=1$ and $\sum_{n \in \mathbb{Z}} \frac{r_{n} m_{n}}{p_{n} \rho_{n}}=0$, then the random walk is recurrent.

Remark 7.6. Let $\sum_{n \in \mathbb{Z}}\left(1 / \rho_{n}\right)<+\infty$ and suppose for example that the $\left(m_{n}\right)_{n \in \mathbb{Z}}$ are a typical realization of some independent uniformly bounded random variables, at least one having a density. Then, almost-surely, the associated random walk is transient. Indeed, the random variable $\omega \longmapsto \sum_{n \in \mathbb{Z}}\left(r_{n} m_{n}(\omega)\right) /\left(p_{n} \rho_{n}\right)$ has a density, so equals 0 with zero probability.

Proof of the proposition \%.5: One can use the recurrence criterion that we have established, but in this situation we fall in a classical context. Indeed, by lemma 4.1, the random walk is recurrent if and only if $\left(T_{n}\right)$ is recurrent with $T_{n}=D_{1}+\cdots+D_{n}$, where the $\left(D_{k}\right)$ are i.i.d. with the same law as $D$, where $D$ is given in (4.4). The point is that $\sigma$ is integrable, thus also $D$. Hence $\mathbb{E}(D) \neq 0$ implies transience because there is a LLN with non-zero speed for $\left(T_{n}\right)$. When $d=1$ and $\mathbb{E}(D)=0$, it is classical that $\left(T_{n}\right)$ is then recurrent (when $d=2$ the situation is more complicated). We leave to the reader the computation, using trees, that $\mathbb{E}(D)=\sum_{n \in \mathbb{Z}}\left(r_{n} m_{n}\right) /\left(p_{n} \rho_{n}\right)$.

Remark 7.7. When $\sum_{n \in \mathbb{Z}} 1 / \rho_{n}<+\infty$, modifying (preserving the $\mathbb{Z}$-invariance) the environment on a single line can change the asymptotics of the random walk (a single $m_{n_{0}}$ can make $\sum_{n \in \mathbb{Z}} r_{n} m_{n} /\left(p_{n} \rho_{n}\right)$ equal 0 or not). This is not true if $\sum_{n \in \mathbb{Z}} 1 / \rho_{n}=+\infty$. As discussed in the introduction of Brémont (2016), in the flat case $p_{n}=q_{n}, n \in \mathbb{Z}$, changing the environment on a single horizontal line did not modify the asymptotics.

7.4. Non-uniform non-elliptic environment on a single line. Taking $d=1$, let us briefly discuss what happens when the medium is $\mathbb{Z}$-invariant (satisfying hypotheses 1.1), except on one line, where ellipticity is also broken. Consider the following example. 
Proposition 7.8. Take at $(m, n)$, with $n \neq 0: p_{n}=q_{n}=r_{n}=1 / 3$, with $\mu_{n}=$ $\delta_{-\operatorname{sign}(n)}$. At $(m, 0)$, with $m \geq 0$, let $p=1$. At $(m, 0)$, with $m<0$, let $q=1$.

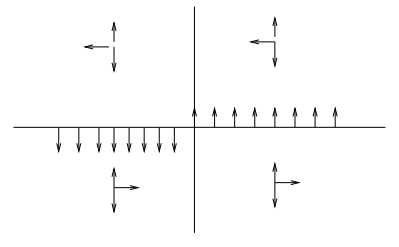

Then this random walk is recurrent.

Remark 7.9. If the environment on the horizontal line $y=0$ is taken for example to be the same as the one of a Northern line, then the random walk in transient. This was proved originally in Campanino and Petritis (2003). This can be seen directly here, as $\Phi_{1}(0, n) \asymp n^{2}$. This gives $\sum 1 / \Phi_{1}(0, n)<+\infty$ and the random walk is transient by proposition 2.5 .

Proof of the proposition 7.8: Consider the sequence of returns on the axis $y=0$. This is a Markov chain. On $\{0,1,2, \cdots\}$, this walk jumps with $-X$, where $X=$ $\sum_{1 \leq k \leq \sigma-1} \Gamma_{k}$ and $P\left(\Gamma_{k}=m\right)=(2 / 3)(1 / 3)^{m}, m \geq 0$. The $\left(\Gamma_{k}\right)$ are independent and $\sigma$ is independent of $\left(\Gamma_{k}\right)$ and has the law of $\min \left\{k \geq 1, S_{k}=0\right\}$, where $\left(S_{k}\right)$ is simple random walk on $\mathbb{Z}$ starting at 0 (with $i . i . d$. jumps with law $\left(\delta_{1}+\delta_{-1}\right) / 2$ ). On $\{-1,-2, \cdots\}$ one jumps with $X$ instead of $-X$.

It is sufficient to restrict this random walk to the subsequence of non-zero jumps. This means replacing the law of $X$ by $\mathcal{L}(X \mid X>0)=\mu$. This is an example of oscillating random walk on $\mathbb{Z}$ in the sense of Kemperman (1974). By theorem 4.8 of Kemperman (1974), this random walk is recurrent if and only if for some $\varepsilon>0$ :

$$
\int_{0}^{\varepsilon}|1-\hat{\mu}(\theta)|^{-2} d \theta=+\infty
$$

Let us examine $\hat{\mu}$ near the origin. Let $p_{0}=\mathbb{P}(X=0)$ and $\chi(\theta)=2 /\left(3-e^{i \theta}\right)$ be the characteristic function of $\Gamma_{1}$. Then:

$$
\hat{\mu}(\theta)=\frac{\mathbb{E}\left(e^{i \theta X} 1_{X>0}\right)}{1-p_{0}}=\frac{\mathbb{E}\left(e^{i \theta X}\right)-p_{0}}{1-p_{0}}=\frac{\mathbb{E}\left(\chi(\theta)^{\sigma-1}\right)-p_{0}}{1-p_{0}} .
$$

Recall that $\mathbb{E}\left(s^{\sigma}\right)=1-\sqrt{1-s^{2}}$, for real $-1<s<1$. This extends analytically to $|z|<1$, using the principal determination of the square root in the complex plane. This gives:

$$
\hat{\mu}(\theta)=\frac{\chi(\theta)^{-1}\left(1-\sqrt{1-\chi(\theta)^{2}}\right)-p_{0}}{1-p_{0}} .
$$

Let $\theta>0$. At the first order, $\chi(\theta)=1+i \theta / 2+O\left(\theta^{2}\right)$. Thus $\sqrt{1-\chi(\theta)^{2}}=$ $\sqrt{\theta} e^{-i \pi / 4}(1+O(\theta))$. Consequently:

$$
\hat{\mu}(\theta)=1-\frac{\sqrt{\theta} e^{-i \pi / 4}}{1-p_{0}}+O(\theta)
$$

This ensures that (7.1) is satisfied. 
7.5. Geometrical interpretation of the criterion. Let us first fix $d=1$. We implicitly use lemma 6.2. Setting $\Phi=\Phi_{1}$ and $\Phi_{+}=\Phi_{1,+}$, as $S_{+}^{0}=\{1\}$, we shall interpret geometrically as a surface in $\mathbb{R}^{2}$ the integral involved in theorem 2.4:

$$
\int_{0<t<1} \frac{\left(\Phi^{-1}(1 / t)\right)^{2}}{\Phi_{+}^{-1}(1 / t)} d t
$$

In $\mathbb{R}^{3}$, consider the canonical basis $\left(e_{1}, e_{2}, e_{3}\right)$, corresponding to coordinates $(X, Y, Z)$. We look at the intervals on the $Y$-axis containing the point 0 , ie all $[-a, b]$, with $a \geq 0$ and $b \geq 0$. We first parametrize points in the half plane $\{X=0, Z>0\}$ with polar coordinates $(\rho, \alpha)$ such that $\rho>0$ and $0 \leq \alpha \leq \pi$ in the following way:

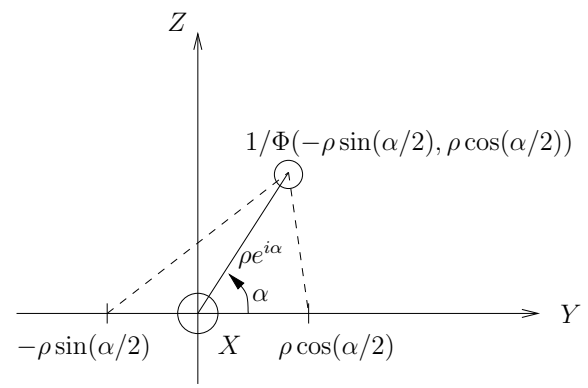

To each $\rho>0$ and $0 \leq \alpha \leq \pi$ we associate the interval $[-\rho \sin (\alpha / 2), \rho \cos (\alpha / 2)]$ containing 0 on the $Y$-axis. We next plug at $\rho e^{i \alpha}$ in the $X$-direction the value $1 / \Phi(-\rho \sin (\alpha / 2), \rho \cos (\alpha / 2))$ (i.e. we take the point $\rho e^{i \alpha}+e_{1} / \Phi(-\rho \sin (\alpha / 2)$, $\rho \cos (\alpha / 2)))$.

This gives a surface above the plane $(Y, Z)$ in the half space $X \geq 0$. We now cut it with the planes $X=t$, for $t>0$, giving some level line $L_{t}$ in the plane $X=t$. Set $\Phi_{++}(n)=\Phi(0, n)$ and $\Phi_{+-}=\Phi(-n, 0)$. Fixing $t>0$, the point on $L_{t}$ with zero $Y$-coordinate has up to constants $\left(t, 0, \Phi^{-1}(1 / t)\right)$ as components. Similarly the extremities of $L_{t}$ have components $\left(t,-\Phi_{+-}^{-1}(1 / t), 0\right)$ and $\left(t, \Phi_{++}^{-1}(1 / t), 0\right)$. Drawing several level lines for some values of $t>0$, the picture is as follows.

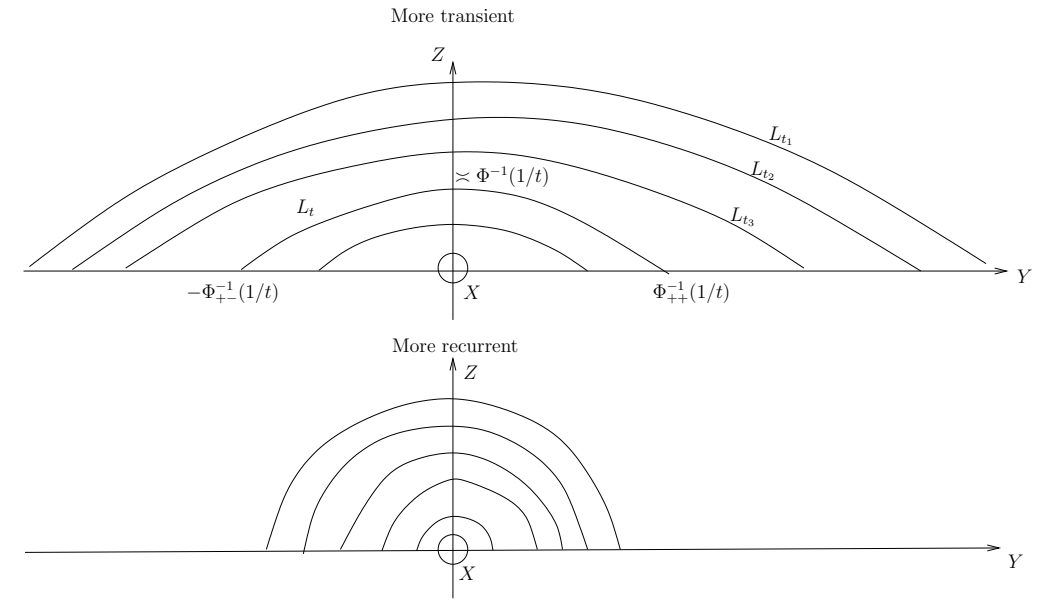

In each plane $X=t$, for $t>0$, we then make some sort of stereographic projections: 


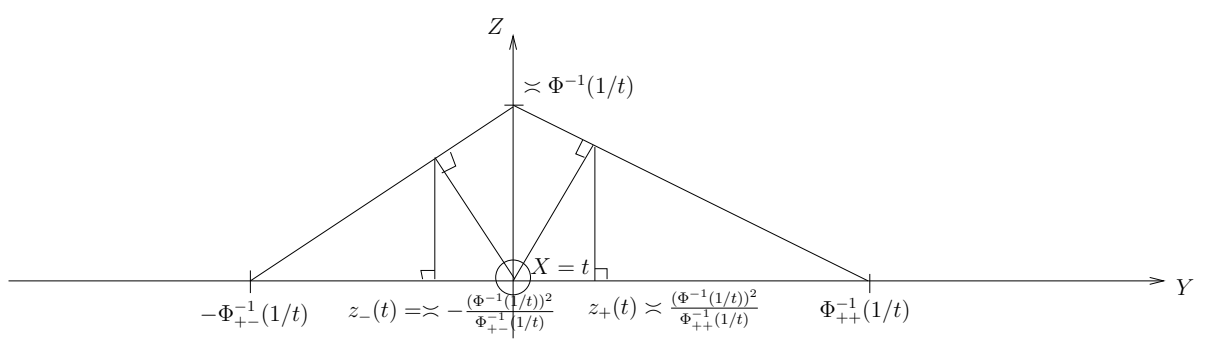

Back to the plane $(X, Y)$, we draw the graphs $\left\{\left(t, z_{+}(t)\right) \mid 0<t<1\right\}$ and $\left\{\left(t, z_{-}(t)\right) \mid 0<t<1\right\}$ and obtain the following picture:

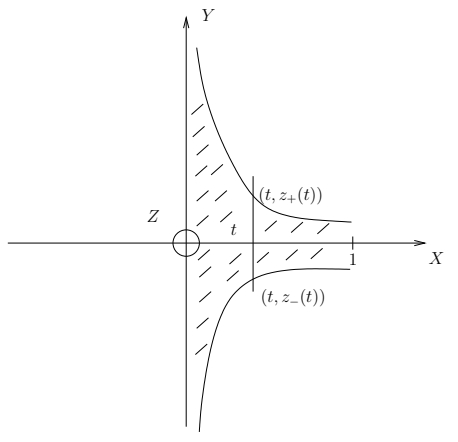

The hatched area above has the same order as (7.2), since the integral can be decomposed into two parts, using the observation that:

$$
\frac{1}{\Phi_{+}^{-1}} \asymp \frac{1}{\Phi_{++}^{-1}}+\frac{1}{\Phi_{+-}^{-1}} .
$$

When $d=2$, we draw for all $u \in S_{+}^{1}$ the same picture in the plane $\left(u, e_{3}\right)$, replacing $e_{1}$ by $u$ and $e_{2}$ by $e_{3}$, using the quantities relative to $\Phi_{u}(-a, b)$. Rotating with respect to $u$ in the half-circle $S_{+}^{1}$ (we have just drawn below the hatched surfaces corresponding to two vectors $u$ and $v$ ), one gets a three-dimensional object naively looking like some half anisotropic pseudosphere. Its volume equals, up to multiplicative constants, the integral appearing in theorem 2.4.

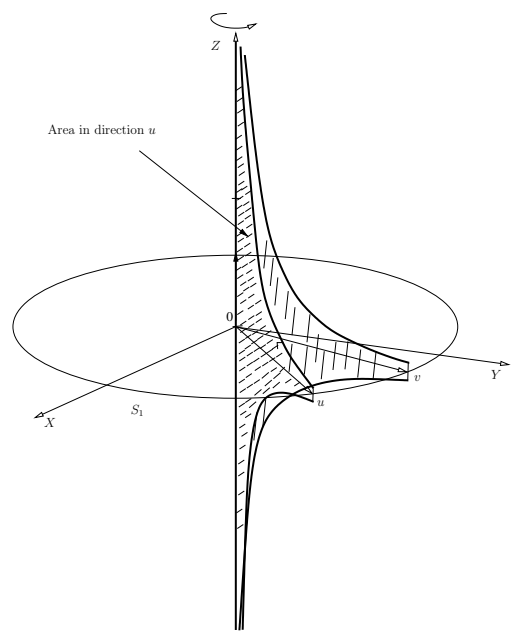


Remark 7.10. When the random walk goes "frankly" in some direction $u \in S^{1}$, then for all $v \in S_{+}^{1}$ not orthogonal to $u$, some pinching effect occurs towards the horizontal plane in the hatched surface in direction $v$, making its area (and thus the global volume) smaller.

7.6. Concluding remarks. Examples more substantial than the ones presented here deserve a specific study. A better understanding of the geometry underlying the recurrence criterion would certainly be helpful. It would be interesting for instance to consider the case when the $\left(p_{n}, q_{n}, r_{n}, m_{n}\right)$ are a typical realization of an i.i.d. process with $m_{n}$ independent of $\left(p_{n}, q_{n}\right)$ and $\mathbb{E}\left(\log \left(p_{n} / q_{n}\right)\right)=0$. Examples of a quasi-periodic nature would also be desirable, for example when $\log \rho_{n}$ is an ergodic sum over an irrational rotation on the circle of a centered function.

In another direction, one can think about the analogous model in $\mathbb{Z} \times \mathbb{Z}^{2}$ with a $\mathbb{Z}$ invariant environment. Restricting to vertical movements, the vertical component is now a random walk in $\mathbb{Z}^{2}$. When the latter is simple random walk in $\mathbb{Z}^{2}$, one may observe that there is not much room for recurrence. Indeed, if the horizontal jump at $(m, n) \in \mathbb{Z} \times \mathbb{Z}^{2}$ is $\varepsilon_{n}$, where the $\varepsilon_{n}$ are \pm 1 , as in the Campanino-Petritis model, rough estimates furnish that for any sequence $\left(\varepsilon_{n}\right)_{n \in \mathbb{Z}^{2}}$ the random walk is transient. When the vertical component is a more general recurrent random walk, this may become very difficult. The main problem concerns the distribution of the local time during an excursion of this random walk. There is no tree-structure behind, as in the one-dimensional case, but a complicated graph with loops.

Related to the latter question, a first step seems to be the model in the plane, where:

$$
\mathbb{P}_{(m, n),(m, n \pm 1)}=1 / 4, \mathbb{P}_{(m, n),(m \pm 1, n)}=p_{ \pm}(m, n) / 2,
$$

with $p_{+}(m, n)+p_{-}(m, n)=1$. The vertical component is recurrent and the subsequence of return times on the horizontal axis is a one-dimensional random walk with unbounded jumps. Very few results are known on such a random walk. They suppose a random environment and an integrable jump (Andjel, 1988), which is not the case here.

Acknowledgments. We wish to thank for useful discussions Alexis Devulder, Jacques Printems and Jon Aaronson.

\section{References}

E. D. Andjel. A zero or one law for one-dimensional random walks in random environments. Ann. Probab. 16 (2), 722-729 (1988). MR929074.

A. Beardon and L. Lorentzen. Approximants of Śleszyński-Pringsheim continued fractions. J. Comput. Appl. Math. 132 (2), 467-477 (2001). MR1840642.

J. Brémont. On planar random walks in environments invariant by horizontal translations. Markov Process. Related Fields 22 (2), 267-309 (2016). MR3561139.

M. Campanino and D. Petritis. Random walks on randomly oriented lattices. Markov Process. Related Fields 9 (3), 391-412 (2003). MR2028220.

M. Campanino and D. Petritis. On the physical relevance of random walks: an example of random walks on a randomly oriented lattice. In Random walks and geometry, pages 393-411. Walter de Gruyter, Berlin (2004). MR2087791. 
M. Campanino and D. Petritis. Type transition of simple random walks on randomly directed regular lattices. J. Appl. Probab. 51 (4), 1065-1080 (2014). MR3301289.

J. H. B. Kemperman. The oscillating random walk. Stochastic Processes Appl. 2, 1-29 (1974). MR0362500.

A. Khintchine. Metrische Kettenbruchprobleme. Compositio Math. 1, 361-382 (1935). MR1556899.

J.-F. Le Gall. Random trees and applications. Probab. Surv. 2, 245-311 (2005). MR2203728.

L. Lorentzen and H. Waadeland. Continued fractions with applications, volume 3 of Studies in Computational Mathematics. North-Holland Publishing Co., Amsterdam (1992). ISBN 0-444-89265-6. MR1172520.

G. Matheron and G. De Marsily. Is transport in porous media always diffusive? a counterexample. Water Resources Research 16 (5), 901-917 (1980). DOI: 10.1029/WR016i005p00901.

F. Spitzer. Principles of Random Walk. Springer-Verlag, New York-Heidelberg, second edition (1976). MR0388547. 